Universitat Politècnica de València

Departamento de Matemática Aplicada

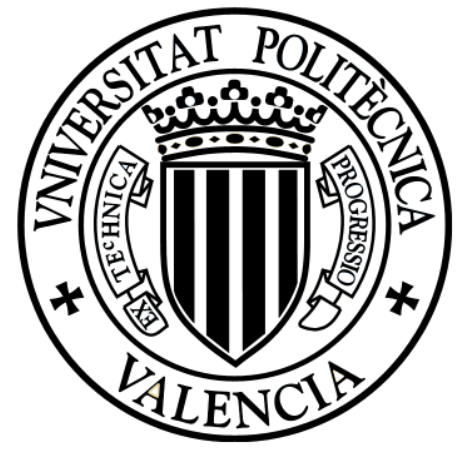

\title{
Generalized fuzzy metric spaces defined by means of continuous t-norms
}

\author{
Memoria presentada por \\ David Miravet Fortuño \\ para optar al Grado de Doctor por la \\ Universitat Politècnica de València \\ Dirigida por los Doctores \\ D. Valentín Gregori Gregori \\ D. Juan José Miñana Prats
}

Gandía, Junio de 2019 

D. Valentín Gregori Gregori, Catedrático del Departamento de Matemática Aplicada de la Universitat Politècnica de València, y D. JuAN José Miñana Prats, Profesor Ayudante Doctor del Departamento de Ciencias Matemáticas e Informática de la Universitat de les Illes Balears

CERTIFICAN: que la presente memoria "Generalized fuzzy metric spaces defined by means of continuous t-norms" ha sido realizada bajo su dirección por D. David Miravet Fortuño, en el Departamento de Matemática Aplicada de la Universitat Politècnica de València, y constituye su tesis para optar al grado de Doctor.

Y para que así conste, presentan la referida tesis, firmando el presente certificado.

Valencia, junio de 2019

Fdo. Valentín Gregori Gregori Fdo. Juan José Miñana Prats 


\section{Agradecimientos}

Me gustaría agradecer a todas las personas que han ayudado de manera directa o indirecta a la realización de esta tesis.

En primer lugar, agradecer a los directores de esta tesis, Valentín Gregori y Juan José Miñana, todo el apoyo recibido y la dedicación y esfuerzo realizado para permitirme avanzar en mi formación como investigador.

En segundo lugar, a mis padres y a mis hermanos por apoyarme en todas las decisiones tomadas a lo largo de mi vida, y especialmente a mi abuela María Rosa, por enseñarme lo maravillosas que pueden ser las matemáticas.

Finalmente, me gustaría agradecer al resto de mi familia, a todos los profesores que han tenido que soportarme, a mis amigos y, en general, a todo aquel que haya formado parte de mi formación, no solamente como matemático, sino como persona. 


\section{RESUMEN INGLÉS}

In 1965, L. Zadeh introduced the concept of fuzzy set, and thus established a new topic of research, known as fuzzy mathematics. Since then, several authors have been investigating the approach of a consistent fuzzy metric space theory. In 1994, George and Veeramani introduced and studied a concept of fuzzy metric space which was a proper modification of the concept given by Kramosil and Michalek. These notions have been studied and developed in several ways during the last 25 years. With the purpose of contributing to the development of the study of the fuzzy theory, in this thesis we have introduced and studied the following items:

(i) We have introduced the concept of extended fuzzy metric $M^{0}$ which is an appropriate extension of a $G V$-fuzzy metric $M$ to $X \times X \times[0,+\infty[$. Furthermore, we have studied convergence and Cauchyness concepts in this context, as well as contractivity and fixed point theorems.

(ii) We have proved the existence of contractive sequences in the sense of D. Mihet in a $G V$-fuzzy metric space which are not Cauchy. Then we have given and studied an appropriate concept of strictly contractive sequence and we have corrected Lemma 3.2 of [12].

(iii) We have introduced and studied a concept of ( $G V$-)fuzzy partial metric space $(X, P, *)$ without any extra conditions on the continuous $t$-norm *. Then we have defined a topology $\mathcal{T}_{P}$ on $X$ deduced from $P$ and we have proved that $\left(X, \mathcal{T}_{P}\right)$ is a $T_{0}$ space.

(iv) We have related the aforementioned notion of $G V$-fuzzy partial metric space with the concept of $G V$-fuzzy quasi-metric space given by Gregori and Romaguera in [24]. A duality is studied by mimicking the techniques used in [6] by Matthews. 


\section{RESUMEN ESPAÑOL}

En 1965, L. Zadeh introdujo el concepto de conjunto fuzzy, estableciendo una nueva línea de investigación, conocida como matemática fuzzy. Desde entonces, varios autores han estado investigando la construcción de una definición consistente de espacio métrico fuzzy. En 1994, George y Veeramani introdujeron y estudiaron un concepto de espacio métrico fuzzy, que era una adecuada modificación del concepto dado por Kramosil y Michalek. Estos conceptos han sido estudiados y desarrollados en diversas líneas durante los últimos 25 años. Con la intención de contribuir a este desarrollo de la teoría fuzzy, en esta tesis hemos introducido y estudiado los siguientes ítems:

(i) Hemos introducido el concepto de espacio métrico fuzzy extendido $M^{0}$, que es una extensión adecuada de una $G V$-métrica fuzzy $M$ a $X \times$ $X \times[0,+\infty[$. Además, hemos estudiado conceptos relacionados con la convergencia y las sucesiones de Cauchy en este contexto, así como teoremas sobre contractividad y punto fijo.

(ii) Hemos probado la existencia de sucesiones contractivas en el sentido de D. Mihet en un espacio métrico fuzzy en el sentido de George y Veeramani que no son de Cauchy. En consecuencia, hemos introducido y estudiado un concepto adecuado de sucesión estrictamente contractiva y hemos corregido el Lema 3.2 de [12].

(iii) Hemos introducido y estudiado una noción de $(G V$-)espacio métrico parcial fuzzy $(X, P, *)$ sin ninguna condición adicional sobre la $t$-norma continua $*$. Después, hemos definido una topología $\mathcal{T}_{P}$ sobre $X$ deducida de $P$ y hemos demostrado que $\left(X, \mathcal{T}_{P}\right)$ es un espacio $T_{0}$.

(iv) Hemos relacionado el mencionado concepto de $G V$-espacio métrico parcial fuzzy con la noción de $G V$-espacio casi-métrico fuzzy definido por 
Gregori y Romaguera en [24]. Se ha estudiado una dualidad entre estos espacios, imitando las técnicas utilizadas por Matthews en [6]. 


\section{RESUMEN VALENCIANO}

En 1965, L. Zadeh va introduir el concepte de conjunt fuzzy, establint una nova línia d'investigació, coneguda com matemàtica fuzzy. Des d'aquell moment, molts autors han investigat la construcció d'una definició consistent d'espai mètric fuzzy. En 1994, George i Veeramani van introduir i estudiar una noció d'espai mètric fuzzy, realitzant una modificació adequada del concepte donat per Kramosil i Michalek. Aquests conceptes han estat estudiats i desenvolupats en diversos sentits durant els últims 25 anys. Amb la intenció de contribuir a aquest desenvolupament de la teoria fuzzy, en aquesta tesi hem introduït i estudiat els següents continguts:

(i) Hem introduït el concepte d'espai mètric extés $M^{0}$, que és una extensió adequada d'una $G V$-mètrica fuzzy $M$ a $X \times X \times[0,+\infty[$. A més, hem estudiat conceptes relacionats amb la convergència i les successions de Cauchy en aquest context, així com teoremes sobre contractivitat i punt fixe.

(ii) Hem provat l'existència de successions contractives en el sentit de D. Mihet en un $G V$-espai mètric fuzzy que no són Cauchy. Conseqüentment, hem aportat i estudiat un concepte apropiat de successió estrictament contractiva i hem corregit el Lema 3.2 de [12].

(iii) Hem introduït i estudiat una noció de $(G V$-)espai mètric parcial fuzzy $(X, P, *)$ sense cap tipus de condició addicional sobre la $t$-norma contínua $*$. A continuació, hem definit una topologia $\mathcal{T}_{P}$ sobre $X$ deduïda de $P$ i hem demostrat que $\left(X, \mathcal{T}_{P}\right)$ es un espai $T_{0}$.

(iv) Hem relacionat el ja mencionat concepte de $G V$-espai mètric parcial fuzzy amb la noció de $G V$-espai quasi-mètric fuzzy definit per Gregori 
i Romaguera en [24]. S'ha estudiat una dualitat entre ambdós espais, imitant les tècniques utilitzades per Matthews en [6]. 


\section{Contents}

$\begin{array}{ll}\text { Introduction } & 5\end{array}$

1 Preliminaries $\quad 15$

1.1 Lattice-ordered monoids and continuous t-norms . . . . . 15

1.2 Fuzzy metric spaces $\ldots \ldots \ldots \ldots$

1.3 Partial metric and quasi-metric spaces . . . . . . . . . . 24

$2 \quad$ Extended fuzzy metric spaces and fixed point theorems $\quad 27$

2.1 Introduction . . . . . . . . . . . . . . . . . 27

2.2 Extended fuzzy metrics $\ldots \ldots \ldots \ldots$

2.3 Topology deduced from an extended fuzzy metric ................... 35 
2.4 Relationship between $\tau_{M^{0} \text {-convergence and }}$ $s$-convergence in extendable fuzzy metrics $\ldots \ldots \ldots 37$

2.5 Cauchyness and completeness . . . . . . . . . . . 42

2.6 Contractivity and fixed point theorems . . . . . . . . 45

3 Contractive sequences in $G V$-fuzzy metric spaces

3.1 Introduction . . . . . . . . . . . . . . . . 51

3.2 Preliminaries . . . . . . . . . . . . . . 54

3.3 Strictly fuzzy contractive sequences . . . . . . . . . . . 55

3.4 Correction to Lemma 3.2 of $[12] \ldots \ldots \ldots$. . . . . . 65

$\begin{array}{lll}4 & \text { Fuzzy partial metric spaces } & 67\end{array}$

4.1 Introduction . . . . . . . . . . . . 67

4.2 Fuzzy partial metric spaces f . . . . . . . . . . . 68

4.3 Topology induced by a fuzzy partial metric . . . . . . . 81

5 A duality relationship between fuzzy partial metrics and $\begin{array}{ll}\text { fuzzy quasi-metrics } & 87\end{array}$

5.1 Introduction . . . . . . . . . . . . . 87 
5.2 From $G V$-fuzzy partial metrics to $G V$-fuzzy

(quasi-)metrics . . . . . . . . . . . . . . 89

5.3 From $G V$-fuzzy quasi-metrics to $G V$-fuzzy

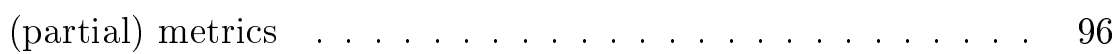

6 Discussion of the obtained results and conclusions

Bibliography

109 


\section{Introduction.}

In 1965, L. Zadeh [54] introduced the notion of fuzzy set as an assignment of a value in $[0,1]$ to each element of a classical, non-empty set $X$. The value is interpreted as the degree of membership of each element to the fuzzy set. Formally, each application $A: X \rightarrow[0,1]$ is called a fuzzy set on $X$. This concept stimulated most of the branches of science, including Mathematics. The first research topic involving fuzzy mathematics were about fuzzy topology. Indeed, C. L. Chang [30] defined a fuzzy topology $\mathcal{T}$ on $X$ as a family of fuzzy sets on $X$ which is closed for unions and for finite intersections.

An interesting and studied topic of research can be found in the obtaining of an appropriate notion of fuzzy metric. The study of metric spaces is based on the notion of distance between points. However, in many real situations this distance cannot be exactly determined. In 1942, K. Menger [32] introduced the concept of probabilistic metric space to approach this problem from the probability theory point of view. Menger proposed to associate a distribution function $F_{x y}$ to each pair of elements $x, y$, and for any positive $t$, interpreted $F_{x y}(t)$ as the probability that the distance from $x$ to $y$ to be less than $t$. These spaces have been widely studied, as it can be seen in $[30,3,27,42]$. 
In 1975, Kramosil and Michalek [29] gave a notion of fuzzy metric space which could be considered as a reformulation, in the fuzzy context, of the notion of probabilistic metric space due to Menger [32]. In this work, by $K M$-fuzzy metric space, or simply, fuzzy metric space $(X, M, *)$ we mean this concept, but in a modern reformulation, due to Grabiec [9], where * is a continuous t-norm (Definition 1.2.2). Later, George and Veeramani [7] introduced a new concept that we will denote $G V$-fuzzy metric space, which constitutes a modification of the concept of $K M$-fuzzy metric space (Definition 1.2.1). We notice that, although these two concepts are not related, many concepts and properties stated for $G V$-fuzzy metrics can be given for $K M$-fuzzy metrics, and vice versa. For this reason, sometimes, the term fuzzy metric, in a wide sense, makes reference to any of them. On the other hand, it is worth to observe that a $G V$-fuzzy metric $M$ can be regarded as a $K M$-fuzzy metric defining $M(x, y, 0)=0$ for all $x, y \in X$. For this reason the concept of $K M$-fuzzy metric can be considered a generalization of the $G V$-fuzzy metric concept, when the value $M(x, y, t)$ does not play any role at $t=0$. In this sense, and if confusion is not possible, we will also refer to $K M$-fuzzy metrics as fuzzy metrics.

Several authors have contributed to the development of this theory, for instance [26, 33, 34, 1, 39, 47, 48]. In particular, it has been proved that the class of topological spaces which are fuzzy metrizable (in its obvious sense) agrees with the class of metrizable topological spaces $[8,21]$ and then, some classical theorems on metric completeness and metric (pre)compactness have been adapted to the realm of fuzzy metric spaces [21]. Nevertheless, the theory of fuzzy metric completion is, in the case of $G V$-fuzzy metrics, very different from the classical theories of metric completion and probabilistic metric completion. In fact, there are $G V$-fuzzy metric spaces which are not completable ([22, Example 2], [23, Example 2] and [20, Example 14]). A characterization of completable $G V$-fuzzy metric spaces was given in [23, 
Theorem 1].

An interesting aspect of this type of fuzzy metrics is that it includes in its definition a parameter $t$. This feature has been successfully used in Engineering applications such as color image filtering [5] and perceptual color differences [16]. From the mathematical point of view it allows to introduce novel (fuzzy) metric concepts that only have natural sense in this fuzzy metric context. This is the case of several concepts of Cauchyness and convergence, related to sequences, appeared in the literature (see [18]).

One can find several generalizations of the concept of metric space in the literature. For instance, in [6] it was introduced the notion of partial metric, a generalized metric for which the self-distance is not necessarily zero. It is worth mentioning that, a partial metric $p$ on a non-empty set $X$ induces a topology $\mathcal{T}(p)$ on $X$ which is $T_{0}$. Interesting notes on it can be seen in [2]. In the same line of research, there is the notion of quasi-metric, which is a classical metric that is not necessarily symmetric. In [6], Matthews stablishes a relationship between partial metrics and a class of quasi-metrics called weighted quasi-metrics.

In [24], Gregori and Romaguera extended the concept of quasi-metric to the fuzzy context. Also, different works have tried to unify both generalizations of classical metric, partial metric and fuzzy one, in a single one notion. For instance, in [53] was given a concept of fuzzy partial metric space $(X, P, *)$, which was defined using the continuous minimum $t$-norm. Furthermore, the authors endowed a fuzzifying topology (see [51]) to the set $X$ deduced from the fuzzy partial metric $P$. Later on, in [52] it was introduced the concept of probabilistic partial metric as a generalization of both fuzzy metrics and partial ones by means of $\Delta^{+}$-valued sets. A probabilistic partial metric is a generalization of the fuzzy partial metric given in [53]. 
Besides, Y. Yue [51] defined the concept of open ball in a probabilistic partial metric space $(X, P, \wedge)$, where $\wedge$ is the minimum $t$-norm, and proved that the family of open balls induces a (classical) topology $\mathcal{T}_{P}$ on $X$. He also proved fixed point theorems on a complete probabilistic partial metric space $(X, P, \wedge)$, and generalized fixed point theorems given in [9] and in [25]. Recently, in [50] the poset $B X$ of formal balls has been studied in these last one spaces, renamed as fuzzy partial metric spaces. Moreover, in [43] has been introduced a concept of partial fuzzy metric which generalizes the concept of strong (non-Archimedean) $G V$-fuzzy metric (see [19,35]), and there were proved fixed point theorems for complete partial fuzzy metric spaces.

The objective of this work is to study topics that concern contractivity, fixed point theorems, partial metric and quasi-metrics in fuzzy setting. The organization of this work consists of five chapters divided in several subsections, and at the end, a list of references is provided. Chapter 1 is dedicated to basic necessaries about lattice-ordered monoids and continuous $t$-norms (Section 1.1), fuzzy metric spaces (Section 1.2), and partial metric and quasimetric spaces (Section 1.3). Chapter 2 is devoted to the study of a certain extension of the concept of $G V$-fuzzy metric. Chapter 3 is devoted to the study of (fuzzy) contractive sequences and Cauchyness concepts in $G V$-fuzzy metric spaces. Chapter 4 is devoted to fuzzy partial metrics and Chapter 5 to the relationship between $G V$-fuzzy partial metrics and $G V$-fuzzy quasimetrics. The chapters contain explanatory notes and appropriate examples, throughout the paper, for illustrating the theory. The contents of these chapters are summarized in the following paragraphs.

A $G V$-fuzzy metric $M$ on $X$ satisfies $M(x, y, t)>0$ for all $x, y \in X$, $t>0$ and it is not defined at $t=0$. For this reason, in a strict sence, $G V$-fuzzy metrics and fuzzy metrics (in the Kramosil and Michalek's sense) are not related. In Chapter 2 we try to relate both concepts in a particular 
case, as follows. We say that a $G V$-fuzzy metric $M$ on $X$ is extendable (Definition 2.2.6) if its (unique) continuous extension $M^{0}$ of $M$ to $X^{2} \times$ $\left[0,+\infty\left[\right.\right.$ is always positive, i.e. $M^{0}(x, y, 0)=\bigwedge_{t>0} M(x, y, t)>0$ for all $x, y \in X$. In such a case $M^{0}$ is called an extended fuzzy metric on $X$, or with more precision, $M^{0}$ is the (fuzzy metric) extension of $M$. The existence of $M^{0}$ is characterized in Theorem 2.2.4. Observe that $M^{0}$ is not a $G V$-fuzzy metric (neither a fuzzy metric), but it behaves, in some sense, as both a $G V$ fuzzy metric space and a fuzzy metric space. In fact, it is possible to construct a topology $\mathcal{T}_{M^{0}}$ on $X$ in a similar way to fuzzy metrics. In Proposition 2.3.3

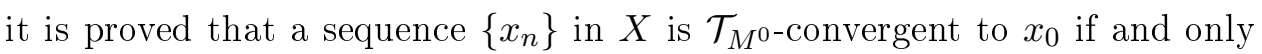
if $\lim _{n} M\left(x_{n}, x_{0}, 0\right)=1$. Hence, the $t$ parameter does not play any role in $\mathcal{T}_{M^{0}}$, and so $M^{0}$ can be studied, from the topological point of view, as a classical metric. It is obvious that $\mathcal{T}_{M} \prec T_{M^{0}}$, and in Section 2.4 it is proved that for an extendable fuzzy metric $M$ we have that $\mathcal{T}_{M}=\mathcal{T}_{M^{0}}$ if and only if $M$ is an $s$-fuzzy metric (i.e. if $\lim _{n} M\left(x_{n}, x_{0}, 1 / n\right)=1$ whenever $\left\{x_{n}\right\}$ is

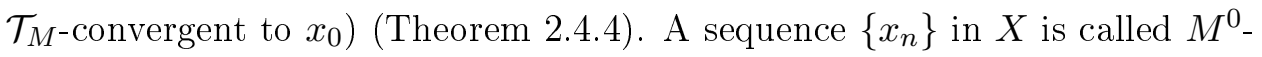
Cauchy if $\lim _{m, n} M^{0}\left(x_{m}, x_{n}, 0\right)=1$ (Definition 2.5.1) and $X$ is $M^{0}$-complete if every $M^{0}$-Cauchy sequence is $M^{0}$-convergent (i.e., $\left\{x_{n}\right\}$ is $\mathcal{T}_{M^{0} \text {-convergent }}$ in $X$ ). Section 2.5 is dedicated to the study of completeness of extended fuzzy metrics. In Definition 2.6.4 is defined for an extendable fuzzy metric $M$ the concept of fuzzy- $\psi-M^{0}$-contractivity, for a mapping $f: X \rightarrow X$, where $\psi$ is a gauge function satisfying the conditions of Definition 2.6.1. In particular, $f$ is called $\psi$-0-contractive if $M(f(x), f(y), 0) \geq \psi(M(x, y, 0))$ for all $x, y \in X$. Example 2.6.6 shows that the $\psi$-0-contractive condition is weaker than the $\psi$ - $M^{0}$-contractive one. Then, for extendable fuzzy metrics it is possible to generalize some existing versions of fixed point theorems, using only the fuzzy $\psi$-0-contractivity. For instance, in Theorem 2.6.10 is stated that every fuzzy $\psi$-0-contractive mapping $f: X \rightarrow X$ of an extendable complete fuzzy metric space $(X, M, *)$ admits a unique fixed point. The results presented in this chapter have been published in [14]. 
In Chapter 3 we study the relationship between Cauchyness and contractivity of sequences in a $G V$-fuzzy metric space $(X, M, *)$. In [25] Gregori and Sapena gave the first concept of a fuzzy contractive sequence ( (ii) of Definition 3.2.3). They formulated the following question, which, up to now, remains open: Is every fuzzy contractive sequence a Cauchy sequence? (Recall that a sequence $\left\{x_{n}\right\}$ is Cauchy if $\lim _{m, n} M\left(x_{m}, x_{n}, t\right)=1$ for all $t>0$ ) (Definition 3.2.1). Several other concepts of (fuzzy) contractive sequence have appeared in the literature which are according to the corresponding concepts of contractive mappings. Here we deal with other three contractivity concepts, which are related to the aforementioned contractivity concept given by Gregori and Sapena ( $G S$-contractivity) due to Romaguera and Tirado ( $R T$-contractivity), Wardowski ( $\mathcal{H}$-contractivity) and Mihet $(\psi$ contractivity) (Definition 3.2.3). They satisfy the following chain of implications:

$R T$-contractive $\rightarrow G S-$ contractive $\rightarrow \mathcal{H}$-contractive $\rightarrow \psi$-contractive

It is easy to prove that every $\psi$-contractive sequence is G-Cauchy $\left(\left\{x_{n}\right\}\right.$ is $G$-Cauchy if $\lim _{n} M\left(x_{n}, x_{n+p}, t\right)=1$ for all $t>0, n=1,2, \ldots$ [10]) (Proposition 3.3.12). In Lemma 3.2 of [12] it is asserted that under a certain condition a $\psi$-contractive sequence is Cauchy. Nevertheless, this lemma is not true as it is pointed out in Example 3.3.13 (the main result of this chapter). Indeed, in this example a non-Cauchy $\psi$-contractive sequence in a $G V$-fuzzy metric space, which fulfills hypotheses of Lemma 3.2 of [12], is constructed. To overcome this inconvenience, we have defined the following concept (Definition 3.3.2): A sequence $\left\{x_{n}\right\}$ is strictly $\psi$-contractive if $M\left(x_{m+1}, x_{n+1}, t\right) \geq \psi\left(M\left(x_{m}, x_{n}, t\right)\right)$ for all $m, n \in \mathbb{N}, t>0$. We notice that this condition is stronger than $\psi$-contractivity, and we extend it to the other contractivity conditions aforementioned. Moreover, this condition is fulfilled by every iterative sequence $\left\{f^{n)}(x)\right\}_{n}, x \in X$, deduced from a contractive mapping $f$ (Proposition 3.3.5), for each one of the contractivity 
concepts studied in this work (Definition 3.3.2). Now, the most interesting fact is that Lemma 3.2 of [12] is true if we replace $\psi$-contractivity by strict $\psi$-contractivity (Proposition 3.3.12). Consequently, fixed point theorems of [12] are correct. The results presented in this chapter have been published in [13].

In Chapter 4 we approach the concept of fuzzy partial metric space $(X, P, *)$. This approach is an extension to the fuzzy setting, in the senses of Kramosil and Michalek (Definition 4.2.1) and George and Veeramani (Definition 4.2.2), of the concept of partial metric due to Matthews. This approach has been made using the residuum operator $\rightarrow_{*}$ associated to a continuous $t$-norm, without any extra condition on $*$. As in the case of fuzzy metrics a $G V$-fuzzy partial metric can be regarded as a fuzzy partial metric defining $P(x, y, 0)=0$ for each $x, y \in X$. Also, a $(G V$-)fuzzy metric is a $(G V$-)fuzzy partial metric satisfying $P(x, x, t)=1$ for all $x \in X, t>0$. Examples of fuzzy partial metric spaces for the minimum, product and Hamacher continuous $t$-norms are given in Examples 4.2.10, 4.2.5 and 4.2.6, respectively. A significative difference with respect to fuzzy metrics is the fact that for a given fuzzy partial metric space $(X, P, *)$ it is not true, in general, that $(X, P, \diamond)$ has to be also a fuzzy partial metric space, whenever $\diamond \leq *$ (Remark 4.2.8). This difference is illustrated in Example 4.2.7. In Section 4.3 we define a topology $\mathcal{T}_{P}$ on $X$ deduced from $P$ when $P$ is a $(G V$-)fuzzy partial metric. To construct $\mathcal{T}_{P}$, the value of $P(x, y, t)$ at $t=0$ does not play any role, as in the fuzzy metric case, and so we suppose that $P$ is a fuzzy partial metric. In Definition 4.3.3, and similarly to the case of fuzzy metrics, it is defined the concept of open ball $B_{P}$ centered at $x \in X$, with radius $r \in] 0,1\left[\right.$ and parameter $t>0$ as $B_{P}(x, r, t)=\left\{y \in X: P_{x, x, y}^{\prime}(t)>1-r\right\}$, where $P_{x, x, y}^{\prime}(t)=\sup \left\{P(x, x, s) \rightarrow_{*} P(x, y, s): s \in\right] 0, t[\}$. This function $P_{x, x, t}^{\prime}$ is non-decreasing and also left-continuous (Lemma 4.3.4), which leads to prove Theorem 4.3.7: the family of open balls in a fuzzy partial metric 
space $(X, P, *)$ forms a base for a topology $\mathcal{T}_{P}$ on $X$, which is $\mathcal{T}_{0}$. We cannot refuse to take the supremum when defining $P_{x, x, y}^{\prime}$, as it is illustrated in Example 4.3.2. Nevertheless, after some explanatory comments (Remark 4.3.9), a question related to the removal of the supremum is posed at the end of the chapter (Question 4.3.8). The results presented in this chapter have been published in [15].

In Chapter 5 we establish a duality between the $G V$-fuzzy quasi-metrics due to Gregori and Romaguera (Definition 5.2.1) and $G V$-fuzzy partial metrics studied in Chapter 4. This duality is motivated by the classical relationship between a partial metric space and a quasi-metric space, which was introduced by Matthews in [6]. In Section 5.2 we provide some examples of $G V$-fuzzy quasi-metric space (Example 5.2.2). Later, we show a way to define a $G V$-fuzzy quasi-metric space from a given $G V$-fuzzy partial metric space $(X, P, *)$, where $*$ is an Archimedean $t$-norm (Theorem 5.2.3). Furthermore, Example 5.2.4 proves that the condition of the $t$-norm being Archimedean is necessary to apply the aforementioned theorem. The end of this section is dedicated to show that the topology of a $G V$-fuzzy partial metric coincides with the topology of its deduced $G V$-fuzzy quasi-metric. Section 5.3 studies the converse problem, i.e. how to define a $G V$-fuzzy partial metric from a $G V$-fuzzy quasi metric. To begin with, we give a definition of $G V$-fuzzy weighted quasi-metric, which is a generalization of the classical weighted quasi-metrics to the fuzzy context (Definition 5.3.1). Also, we illustrate this definition with two examples (Propositions 5.3.2 and 5.3.3). Analogously to the previous section, we stablish a way to define a $G V$-fuzzy partial metric space from a $G V$-fuzzy weighted quasi-metric space (Theorem 5.3.4). Making use of a previous lemma, we prove that, as we point out in Theorem 5.2.3, the $t$-norm has to be Archimedean (Example 5.2.4). To conclude this chapter, we show, analogously to Section 5.2, that the topology of a $G V$ fuzzy weighted quasi-metric space coincides with the topology of its deduced 
$G V$-fuzzy partial metric space. Moreover, the $G V$-fuzzy quasi-metric space deduced from a $G V$-fuzzy partial metric space is weightable (Theorem 5.3.7). 


\section{Chapter 1}

\section{Preliminaries}

\subsection{Lattice-ordered monoids and continuous $t$-norms}

In this section, we will recall some notions and results related to latticeordered monoids. They will be useful in order to introduce a binary operator that we will use in our definition of fuzzy partial metric space in Chapters 4 and 5. We begin recalling some concepts introduced in [37].

Definition 1.1.1. Let $(L, \preceq)$ be a lattice and let $(L, *)$ be a semigroup with neutral element, denoted by 1 .

(i) The triple $(L, *, \preceq)$ is called a lattice-ordered monoid (or an $l$-monoid) if for all $x, y, z \in L$ we have

(LM1) $x *(y \vee z)=(x * y) \vee(x * z)$,

(LM1) $(x \vee y) * z=(x * z) \vee(y * z)$.

(ii) An $l$-monoid $(L, *, \preceq)$ is said to be commutative if the semigroup $(L, *)$ 
is commutative.

(iii) A commutative $l$-monoid $(L, *, \preceq)$ is called a commutative residuated l-monoid if there exists a further binary operation $\rightarrow_{*}$ on $L$ such that for each $x, y, z \in L$ we have

$$
x * y \preceq z \text { if and only if } x \preceq y \rightarrow_{*} z .
$$

In this case, $\rightarrow_{*}$ is called the $*$-residuum. In [37] it was observed that for each commutative residuated $l$-monoid $(L, *, \preceq)$, the $*$-residuum operator $\rightarrow_{*}$ is uniquely determined by the formula

$$
x \rightarrow_{*} y=\sup \{z \in L: x * z \preceq y\}
$$

(iv) An $l$-monoid $(L, *, \preceq)$ is called integral if there is a greatest element in the lattice $(L, \preceq)$ which coincides with the neutral element of the semigroup $(L, *)$.

(v) A commutative integral $l$-monoid $(L, * \preceq)$ is called divisible if for each $x, y \in L$ with $y \preceq x$ there exists $z \in L$ such that $x * z=y$.

The concepts introduced in Definition 1.1.1 are very related with the notion of triangular norm (briefly, $t$-norm). Recall that a $t$-norm is a binary operation $*$ on the unit interval $[0,1]$ such that it is commutative, associative, monotone, and satisfies that $x * 1=x$ for every $x \in[0,1]$.

The most commonly used continuous $t$-norms in Fuzzy Logic are the minimum, denoted by $\wedge$, the usual product, denoted by $*_{P}$, and the Lukasievicz $t$-norm, denoted by $* \mathfrak{L}$, where $x * \mathfrak{L} y=\max \{0, x+y-1\}$.

The aforementioned relation was established in the following proposition provided in [37]. 
Proposition 1.1.2. For each binary operation $*:[0,1] \times[0,1] \rightarrow[0,1]$ the following are equivalent:

(i) $([0,1], *, \leq)$ is a commutative residuated integral l-monoid.

(ii) $*$ is a left-continuous $t$-norm.

Moreover, in [37] it was pointed out the next corollary of the last result.

Corollary 1.1.3. $*$ is a continuous $t$-norm if and only if $([0,1], *, \leq)$ is a commutative residuated divisible integral l-monoid.

Taking into account the formula (1.1), an immediate consequence of the last corollary is that the $*$-residuum operator $\rightarrow_{*}$ of a continuous $t$-norm $*$ is uniquely determined now by the formula

$$
x \rightarrow_{*} y= \begin{cases}1, & \text { if } x \leq y \\ \sup \{z \in L: x * z=y\}, & \text { if } x>y\end{cases}
$$

Attending to this last expression, the $*$-residuum operator of the minimum, the usual product and the Lukasievicz $t$-norms, respectively, are the following:

$$
\begin{gathered}
x \rightarrow_{\wedge} y= \begin{cases}1, & \text { if } x \leq y ; \\
y, & \text { if } x>y .\end{cases} \\
x \rightarrow_{*_{P}} y= \begin{cases}1, & \text { if } x \leq y ; \\
\frac{y}{x}, & \text { if } x>y .\end{cases} \\
x \rightarrow_{*_{\mathfrak{L}}} y= \begin{cases}1, & \text { if } x \leq y ; \\
1+y-x, & \text { if } x>y .\end{cases}
\end{gathered}
$$

In [37] it was also established a representation theorem of a (large) class of $t$-norms, which are the Archimedean and continuous ones. Recall that 
a $t$-norm is called Archimedean if for each $x, y \in[0,1]$ there exists $n \in \mathbb{N}$ with $x * \cdots * *^{(n)} x<y$, where $* \cdots *^{(n)}$ denotes the $n$-times composition by *. Two well-known examples of Archimedean $t$-norms are the usual product and the Lukasievicz one. Nevertheless, the minimum $t$-norm is an example of non-Archimedean one.

In order to obtain the representation theorem of the Archimedean $t$ norms, it was introduced the following concept.

Definition 1.1.4. An additive generator $f_{*}:[0,1] \rightarrow[0, \infty]$ of a $t$-norm $*$ is a strictly decreasing function which is right-continuous at 0 , satisfying $f_{*}(1)=0$, and such that for $x, y \in[0,1]$ we have

$$
f_{*}(x)+f_{*}(y) \in \operatorname{Ran}\left(f_{*}\right) \cup\left[f_{*}(0), \infty\right]
$$

and also

$$
x * y=f_{*}^{(-1)}\left(f_{*}(x)+f_{*}(y)\right), \text { for all } x, y \in[0,1],
$$

where $f_{*}^{(-1)}$ denotes the pseudo-inverse of the function $f_{*}$ (see [37]).

Now, we present the announced representation theorem.

Theorem 1.1.5. A binary operator $*$ in $[0,1]$ is a continuous Archimedean $t$-norm if and only if there exists a continuous additive generator $f_{*}$ of $*$.

Moreover, an additive generator $f_{*}$ of a continuous Archimedean $t$-norm * allows us to obtain a simpler formula of the *-residuum, as follows:

$$
x \rightarrow_{*} y=f_{*}^{(-1)}\left(\max \left\{f_{*}(y)-f_{*}(x), 0\right\}\right)
$$

In the preceding formula it is assumed that $\infty-\infty=0$ and $\infty-a=\infty$ for each $a \in[0, \infty[$. 
Note that the pseudo-inverse of a continuous additive generator $f_{*}$ is given by

$$
f_{*}^{(-1)}(y)=f_{*}^{-1}\left(\min \left\{f_{*}(0), y\right\}\right) .
$$

Remark 1.1.6. Attending to this last equation, it is obvious that given a continuous Archimedean $t$-norm $*$, its $*$-residuum is continuous on $] 0,1] \times] 0,1]$. Moreover, the $*$-residuum is left-continuous on $[0,1] \times[0,1]$. Nevertheless, one can easily observe in Equation 1.3 that the last affirmation is not true, in general, when we consider continuous non-Archimedean $t$-norms, as it is the minimum $t$-norm.

Corollary 1.1.7. Let $*$ be a continuous Archimedean t-norm, and let $f_{*}$ be its continuous additive generator. Then, for every $a>0$, we have that $f_{*}^{(-1)}\left(f_{*}(a)\right)=a$.

To finish this subsection, we recall another well-known continuous Archimedean $t$-norm called the Hamacher product. It will be denoted by $*_{H}$ and it is given by the following expression:

$$
a *_{H} b= \begin{cases}0, & \text { if } a=b=0 ; \\ \frac{a b}{a+b-a b}, & \text { elsewhere }\end{cases}
$$

for each $a, b \in[0,1]$.

In [37] it was pointed out that the function $f_{H}(x)=\frac{1-x}{x}$ is an additive generator of $*_{H}$ and so, on account of formula (1.8), the function $f_{H}^{(-1)}(y)=$ $\frac{1}{1+y}$ is its pseudo-inverse. Attending to these observations and taking into

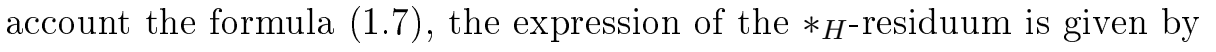

$$
x \rightarrow_{*_{H}} y= \begin{cases}1, & \text { if } x \leq y \\ \frac{x y}{x y+x-y}, & \text { if } x>y\end{cases}
$$




\section{$1.2 \quad$ Fuzzy metric spaces}

Definition 1.2.1. (George and Veeramani [7]). A $G V$-fuzzy metric space is an ordered triple $(X, M, *)$ such that $X$ is a (non-empty) set, $*$ is a continuous $t$-norm and $M$ is a fuzzy set on $X \times X \times] 0, \infty[$ satisfying the following conditions, for all $x, y, z \in X, s, t>0$ :

(GV1) $M(x, y, t)>0$

(GV2) $M(x, y, t)=1$ if and only if $x=y$;

(GV3) $M(x, y, t)=M(y, x, t)$

(GV4) $M(x, y, t) * M(y, z, s) \leq M(x, z, t+s)$;

$\left.\left.(\mathrm{GV} 5) M\left(x, y, \_\right):\right] 0, \infty[\rightarrow] 0,1\right]$, also denoted as $M_{x y}$, is continuous.

The axiom (GV1) is justified by the authors because in the same way that a classical metric does not take the value $\infty$ then $M$ cannot take the value 0 . The axiom (GV2) is equivalent to the following:

$$
M(x, x, t)=1 \text { for all } x \in X, t>0 \text { and } M(x, y, t)<1 \text { for all } x \neq y, t>0 \text {. }
$$

The axiom (GV2) gives the idea that only when $x=y$ the degree of nearness of $x$ and $y$ is perfect, or simply 1 , and then $M(x, x, t)=1$ for each $x \in X$ and for each $t>0$. In this manner the values 0 and $\infty$ in the classical theory of metric spaces are identified with 1 and 0 , respectively, in this fuzzy theory. Axioms (GV3) and (GV4) are a fuzzy version of the symmetry and the triangular inequality, respectively. Finally, in (GV5) the authors only assume that the variable $t$ behave nicely, that is, they assume that for fixed $x$ and $y$, the function $t \rightarrow M(x, y, t)$ is continuous without any imposition for $M$ as $t \rightarrow \infty$. 
If $(X, M, *)$ is a $G V$-fuzzy metric space, we will say that $(M, *)$ is a $G V$-fuzzy metric on $X$. Also, if confusion is not possible, we will say that $(X, M)$ is a $G V$-fuzzy metric space or $M$ is a $G V$-fuzzy metric on $X$. This terminology will be also extended along this work in other concepts, as usual, without explicit mention.

Definition 1.2.2. (Kramosil and Michalek, [29]) A $K M$-fuzzy metric space (or, simply, fuzzy metric space) is an ordered triple $(X, M, *)$ such that $X$ is a non-empty set, $*$ is a continuous $t$-norm and $M$ is a fuzzy set on $X \times X \times[0, \infty[$ that satisfies (GV3) and (GV4), and

(KM1) $M(x, y, 0)=0$;

(KM2) $M(x, y, t)=1$ for all $t>0$ if and only if $x=y$;

$(\mathrm{KM} 5) M\left(x, y, \_\right):[0, \infty[\rightarrow[0,1]$ is left continuous.

We will also say that $(M, *)$, or simply $M$, is a $(K M$-)fuzzy metric on $X$.

Lemma 1.2.3. (Grabiec [9]) Let $(X, M, *)$ be a fuzzy metric space. Then, the real function $M\left(x, y,{ }_{-}\right)$is non-decreasing for all $x, y \in X$.

Remark 1.2.4. If $(X, M, *)$ is a $(G V$-)fuzzy metric space, and $\diamond$ is a continuous $t$-norm such that $\diamond \leq *$, we have that $(X, M, \diamond)$ is also a $(G V$-)fuzzy metric space.

It is worth noting that, by defining the probabilistic metric $F_{x y}(t)=$ $M(x, y, t)$, every $K M$-fuzzy metric space $(X, M, *)$ becomes a generalized Menger space, [38], under the continuous $t$-norm $*$. On the other hand a $G V$-fuzzy metric space can be considered a $K M$-fuzzy metric space if we extend $M$ defining $M(x, y, 0)=0$ for all $x, y \in X$. For this reason, and if 
confusion is not possible, by fuzzy metric we mean both $G V$-fuzzy metric and $K M$-fuzzy metric.

George and Veeramani proved in [7] that every $G V$-fuzzy metric $M$ on $X$ generates a topology $\mathcal{T}_{M}$ on $X$ which has as a base the family of open sets of the form $\left\{B_{M}(x, \epsilon, t): x \in X, 0<\epsilon<1, t>0\right\}$, where $B_{M}(x, \epsilon, t)=\{y \in$ $X: M(x, y, t)>1-\epsilon\}$ for all $x \in X, \epsilon \in] 0,1[$ and $t>0$. The same is valid for $K M$-fuzzy metric spaces.

Example 1.2.5. Let $(X, d)$ be a metric space and let $M_{d}$ a function on $X \times X \times] 0, \infty[$ defined by

$$
M_{d}(x, y, t)=\frac{t}{t+d(x, y)}
$$

Then $\left(X, M_{d}, \wedge\right)$ is a $G V$-fuzzy metric space [7] and $M_{d}$ is called the standard fuzzy metric induced by $d$. The topology $\mathcal{T}_{M_{d}}$ coincides with the topology on $X$ deduced from $d$.

Example 1.2.6. Let $(X, d)$ be a metric space and let $M_{e}$ a function on $X \times X \times] 0, \infty[$ defined by

$$
M_{e}(x, y, t)=e^{-\frac{d(x, y)}{t}}
$$

Then $\left(X, M_{e}, \wedge\right)$ is a $G V$-fuzzy metric space. The topology $\mathcal{T}_{M_{e}}$ coincides with the topology on $X$ deduced from $d$.

Remark 1.2.7. As $\left(X, M_{d}, \wedge\right)$ and $\left(X, M_{e}, \wedge\right)$ are $G V$-fuzzy metric spaces, we can conclude that $\left(X, M_{d}, *\right)$ and $\left(X, M_{e}, *\right)$ are $G V$-fuzzy metric spaces for every continuous $t$-norm $*$.

Definition 1.2.8. A fuzzy metric $M$ on $X$ is said to be stationary [23] if $M$ does not depend on $t$, i.e. if for each $x, y \in X$, the function $M_{x, y}(t)=$ $M(x, y, t)$ is constant. In this case we write $M(x, y)$ instead of $M(x, y, t)$. 
Proposition 1.2.9. (George and Veeramani [7]). A sequence $\left\{x_{n}\right\}$ in a $G V$ fuzzy metric space $(X, M, *)$ converges to $x$ if and only if $\lim _{n} M\left(x_{n}, x, t\right)=$ 1 , for all $t>0$.

Definition 1.2.10. (George and Veeramani [7], Schweizer and Sklar [42]). A sequence $\left\{x_{n}\right\}$ in a $G V$-fuzzy metric space $(X, M, *)$ is said to be $M$-Cauchy if for each $\epsilon \in] 0,1\left[\right.$ and each $t>0$ there is $n_{0} \in \mathbb{N}$ such that $M\left(x_{n}, x_{m}, t\right)>1-\epsilon$ for all $n, m \geq n_{0}$. Equivalently, $\left\{x_{n}\right\}$ is $M$-Cauchy if $\lim _{n, m} M\left(x_{n}, x_{m}, t\right)=1$ for each $t>0$, where $\lim _{n, m}$ denotes the double limit as $n \rightarrow \infty$, and $m \rightarrow \infty$. If confusion is not possible we will say, simply, that $\left\{x_{n}\right\}$ is Cauchy. $\mathrm{X}$ is called $(M-)$ complete if every Cauchy sequence in $X$ is convergent with respect to $\mathcal{T}_{M}$. In such a case $M$ is also said to be complete.

In [17], the authors introduced a stronger concept than convergence:

Definition 1.2.11. Let $(X, M, *)$ be a $G V$-fuzzy metric space. We will say that a sequence $\left\{x_{n}\right\}$ in $X$ is $s$-convergent to $x_{0} \in X$ if

$$
\lim _{n} M\left(x_{n}, x_{0}, \frac{1}{n}\right)=1 .
$$

Moreover, we will say that $(X, M, *)$ is an $s$-fuzzy metric space or simply, that $M$ is an $s$-fuzzy metric, if every convergent sequence is $s$-convergent.

Definition 1.2.12. Let $(X, M, *)$ be a fuzzy metric space. The fuzzy metric $M$ (or the fuzzy metric space $(X, M, *)$ ) is said to be strong if it satisfies for each $x, y, z \in X$ and each $t>0$ the following:

$$
M(x, z, t) \geq M(x, y, t) * M(y, z, t) . \quad\left(G V 4^{\prime}\right)
$$

Following terminology of probabilistic metric spaces, [10, 28], some authors call non-Archimedean fuzzy metrics those that also satisfy equation (GV4'). Notice that in this case there is not any correspondence, in the 
above sense, between non-Archimedean metrics and non-Archimedean fuzzy metrics since $M_{d}$ always satisfies $M_{d}(x, z, t) \geq M_{d}(x, y, t) \cdot M_{d}(y, z, t)$ and also because all stationary fuzzy metrics would be non-Archimedean. Further (GV4') is not stronger than (GV4) and it means that if we replace (GV4) by (GV4') then $M$ could not be a fuzzy metric on $X$. (Indeed, $M(x, y, t)=\frac{1 / t}{1 / t+d(x, y)}$ satisfies (GV1)-(GV3), (GV4') and (GV5) and it does not satisfies (GV4).)

\subsection{Partial metric and quasi-metric spaces}

In this subsection we recall two generalizations of the notion of classical metric space. First, we recall the concept of quasi-metric space.

Definition 1.3.1. A quasi-metric space is a pair $(X, q)$ where $X$ is a nonempty set, and $q: X^{2} \rightarrow[0,+\infty[$ is a mapping such that

(Q1) $q(x, y)=q(y, x)=0$ if and only if $x=y$ for every $x, y \in X$.

(Q2) $q(x, z) \leq q(x, y)+q(y, z)$, for each $x, y, z \in X$.

We also say that $q$ is a quasi-metric on $X$.

In a similar way that a classical metric, given a quasi-metric space $(X, q)$, then $q$ induces a $T_{0}$ topology $\mathcal{T}(q)$ on $X$ which has as a base the family of open balls $\left\{B_{q}(x ; \epsilon): x \in X, \epsilon>0\right\}$, where $B_{q}(x ; \epsilon)=\{y \in X: q(x, y)<\epsilon\}$, for each $x \in X, t>0$.

Another well-known generalization of metric space was introduced by Matthews in [6]. It was defined as follows. 
Definition 1.3.2. (see [6]) A partial metric space is a pair $(X, p)$ where $X$ is a non-empty set, and $p: X^{2} \rightarrow[0,+\infty[$ is a mapping such that, for each $x, y, z \in X$, the following conditions are satisfied:

(P1) $p(x, x)=p(x, y)=p(y, y)$ if and only if $x=y$.

(P2) $p(x, x) \leq p(x, y)$.

(P3) $p(x, y)=p(y, x)$.

(P4) $p(x, z) \leq p(x, y)+p(y, z)-p(y, y)$.

We also say that $p$ is a partial metric on $X$.

Also, Matthews showed in [6] that a partial metric $p$ on a non-empty set $X$ induces a $T_{0}$ topology $\mathcal{T}(p)$ on $X$ which has as a base the family of open balls $\left\{B_{p}(x ; \epsilon): x \in X, \epsilon>0\right\}$, where $B_{p}(x ; \epsilon)=\{y \in X: p(x, y)-p(x, x)<\epsilon\}$, for each $x \in X, t>0$.

Remark 1.3.3. Note that axiom (P4) can be rewritten as

$$
q(x, z)-q(x, x) \leq p(x, y)-p(x, x)+p(y, z)-p(y, y) .
$$

This equation is more appropriate to establish a duality between quasimetrics and partial metrics.

Remark 1.3.4. Note that the unique difference between a quasi-metric and a classical metric is the symmetry since, in general, $q(x, y) \neq q(y, x)$. On the other hand, a partial metric does not satisfy, in general, that $p(x, x)=0$ for each $x \in X$. 


\section{Chapter 2}

\section{Extended fuzzy metric spaces and fixed point theorems}

\section{$2.1 \quad$ Introduction}

A significant characteristic of a fuzzy metric is that it contains in its definition a parameter $t$. Related to it, we focus our attention in two facts about $M$. First, axiom (GV1) demands that $M(x, y, t)>0$ for all $x, y \in X$ and $t>0$, which is in accordance to classical metrics that do not take the value $+\infty$. Second, axiom (GV5) requires that $\left.\left.M_{x, y}:\right] 0,+\infty[\rightarrow] 0,1\right]$ be a continuous function, where $M_{x, y}(t)=M(x, y, t)$. These two facts suggest the introduction of a new notion of fuzzy metric $M^{0}$, which we study in this chapter.

Stationary fuzzy metrics are the closest to classical ones. It is clear that the expression of a stationary fuzzy metric $M$ on $X$ can be regarded as a 
fuzzy set $M^{0}$ on $X^{2} \times\left[0,+\infty\right.$ [ given by $M^{0}(x, y, t)=M(x, y)$ satisfying the two facts aforementioned, i.e., $M^{0}(x, y, t)>0$ and $M_{x, y}^{0}:[0,+\infty[\rightarrow] 0,1]$ is continuous for $x, y \in X$ and $t \geq 0$.

The above paragraph suggests to consider fuzzy sets $M^{0}: X^{2} \times[0,+\infty[\rightarrow$ ]0,1] satisfying all axioms of a fuzzy metric, but currently for $t \geq 0$. The tern $\left(X, M^{0}, *\right)$ will be called extended fuzzy metric space (Definition 2.2.1) and the aim of Section 2.2 is the study of these spaces. First, we are interested in the relationship between fuzzy metrics and extended fuzzy metrics. Theorem 2.2.4 shows that every extended fuzzy metric $M^{0}$ is a natural extension of those fuzzy metrics, called extendable, which satisfy that $\bigwedge_{t>0} M(x, y, t)>$ 0 , and vice versa. The natural extension is $M^{0}(x, y, 0)=\bigwedge_{t>0} M(x, y, t)$, for all $x, y \in X$. It is now, a natural process to adapt the concepts of fuzzy metrics to extended fuzzy metrics.

In Section 2.3, we introduce and generalize the concept of open ball $B_{M^{0}}(x, r, t)$ (Definition 2.3.1) and then, we prove that the family $\left\{B_{M^{0}}(x, r, 0): x \in X, r \in\right] 0,1[\}$ is a base for a topology $\tau_{M^{0}}$ on $X$ finer than $\tau_{M}$, which is called the topology generated by $M^{0}$. This result is obtained after observing that $\left(N_{M}, *\right)$ is a stationary fuzzy metric on $X$, where $N_{M}(x, y)=\bigwedge_{t>0} M(x, y, t)$ for all $x, y, \in X$. Then, it is easy to conclude that $\tau_{M^{0}}=\tau_{N_{M}}$ and hence $\tau_{M^{0}}$ is metrizable. Furthermore, we pointed out that a sequence $\left\{x_{n}\right\}$ converges to $x_{0}$ in $\tau_{M^{0}}$ if and only if $\lim _{n} M^{0}\left(x_{n}, x_{0}, 0\right)=1$ (Proposition 2.3.3). From the topological point of view (Remark 2.3.4), the class of extended fuzzy metrics $\left(X, M^{0}, *\right)$ are so close to metrics that topological results related to $M^{0}$ can be established as a simple extension of classical concepts to the fuzzy setting, only by modifying the notation.

In Section 2.4, after proving that $M^{0}: X^{2} \times[0,+\infty[\rightarrow] 0,1]$ is continuous (Proposition 2.4.1), we characterize those extendable fuzzy metrics in which 
$\tau_{M}=\tau_{M^{0}}$ (Theorem 2.4.4). Such spaces are the so called $s$-fuzzy metrics. Besides, this characterization motivates a study in the relationship between $\tau_{M^{0} \text {-convergence and } s \text {-convergence. }}$

With respect to completeness, although there are many concepts of Cauchy sequence in the literature (see [18]), in Section 2.5 we only pay attention to the original concept of Cauchy sequence given by H. Sherwood in $P M$-spaces [45]. It was adapted later by George and Veeramani to the fuzzy metric context (Definition 1.2.10). Then, an adaptation to the extended context of completeness and $M$-Cauchyness concepts is given. Furthermore, we provide some properties and observations on $M^{0}$-Cauchyness and $M^{0}$-completeness. For instance, $M^{0}$ is complete if and only if $N_{M}$ is complete (Proposition 2.5.6). Also, if $M$ is an $s$-fuzzy metric, we have that, if $M$ is complete, then $M^{0}$ is complete (Proposition 2.5.7). However, the converse is false (Example 2.5.8).

The last topic that we approach, in Section 2.6, is contractivity, which plays a crucial role in fixed point theory. It should be expected that in fuzzy metrics with strong properties one should be able to weaken the usual contractive conditions in order to ensure the existence of fixed points, for a larger class of contractive mappings. Indeed, this is so. First we notice that the condition itself of being $M$ extendable is used explicitly [12, Theorem 3.3], or in a concealed or relaxed way in order to obtain fixed point theorems ([49, Theorem 3.2] or [36, Theorem 2.4]). We here go further and we will give a notion of $\psi$-0-contractive mapping (Definition 2.6.7), that is, contractivity assumed only at $t=0$. Then, we prove that there are $\psi$-0-contractive mappings in $\left(X, M^{0}, *\right)$ which are not $\psi$-contractive in $(X, M, *)$ (Example 2.6.6). Then, mimicking arguments in the literature one can give fixed point theorems for extendable fuzzy metrics in a more general version. It is the case of Theorem 2.6.10. The reader can find in this example a method for 
obtaining more general results in fixed point theory, but for extendable fuzzy metrics.

\subsection{Extended fuzzy metrics}

We begin this section introducing the announced concept of extended fuzzy metric space.

Definition 2.2.1. The tern $\left(X, M^{0}, *\right)$ is called an extended fuzzy metric space if $X$ is a (non-empty) set, $*$ is a continuous $t$-norm and $M^{0}$ is a fuzzy set on $X^{2} \times[0,+\infty$ [ satisfying the following conditions, for each $x, y, z \in X$ and $t, s \geq 0$

(EFM1) $M^{0}(x, y, t)>0$;

(EFM2) $M^{0}(x, y, t)=1$ if and only if $x=y$;

(EFM3) $M^{0}(x, y, t)=M^{0}(y, x, t)$;

(EFM4) $M^{0}(x, y, t) * M^{0}(y, z, s) \leq M^{0}(x, z, t+s)$;

(EFM5) $M_{x, y}^{0}:[0,+\infty[\rightarrow] 0,1]$ is continuous, where $M_{x, y}^{0}(t)=M^{0}(x, y, t)$.

It is also said that $\left(M^{0}, *\right)$, or simply $M^{0}$, is an extended fuzzy metric on $X$. If $\star$ is a continuous $t$-norm satisfying $\star \leq *$ then $\left(M^{0}, \star\right)$ is also an extended fuzzy metric on $X$.

Remark 2.2.2. Recently, in [31] it was introduced the concept of extended fuzzy $b$-metric space, with the aim of generalizing the notion of fuzzy $b$-metric space. Both notions generalize the concept of fuzzy metric by means of relaxing the triangle inequality. Nevertheless, the goal of introducing Definition 
2.2.6 is to extend the domain of the $t$ parameter to $[0,+\infty[$, in the concept of the $G V$-fuzzy metric. Notice that the concept of extended fuzzy $b$-metric, introduced in [31], is not related with this new concept introduced above.

After introducing this new concept, we present some examples of it.

Example 2.2.3. (a) If $M$ is a stationary fuzzy metric on $X$, then $M^{0}(x, y, t)=M(x, y)$ for all $x, y \in X$ and $t \geq 0$ is, obviously, an extended fuzzy metric on $X$, for the same $t$-norm. Since, again, $t$ does not play any role in the definition of $M^{0}$, we also say that $M^{0}$ is stationary. Further, since in this case the expression of $M$ can be regarded itself as an extended fuzzy metric on $X$, we will not distinguish between $M$ and $M^{0}$, if confusion is not possible.

(b) Let $X$ be the interval $] 0,+\infty\left[\right.$ and define the fuzzy set $M^{0}$ on $X^{2} \times$ $\left[0,+\infty\left[\right.\right.$ by $M^{0}(x, y, t)=\frac{\min \{x, y\}+t}{\max \{x, y\}+t}$. Then $M^{0}$ is an extended fuzzy metric on $X$, for the product $t$-norm.

(c) Let $(X, d)$ be a metric space and let $\phi:[0,+\infty[\rightarrow] 0,1]$ be a nondecreasing continuous function with $\phi(0)>0$. Then $\left(X, M_{\phi}^{0}, *\right)$ is an extended fuzzy metric, where $*$ is the product $t$-norm and $M_{\phi}^{0}(x, y, t)=$ $\frac{\phi(t)}{\phi(t)+d(x, y)}$, for all $x, y \in X$ and $t \geq 0$.

The following theorem shows the relationship between fuzzy metrics and extended fuzzy metrics that one can observe in the last example.

Theorem 2.2.4. Let $M$ be a fuzzy set on $\left.X^{2} \times\right] 0,+\infty\left[\right.$, and denote by $M^{0}$ its extension to $X^{2} \times\left[0,+\infty\left[\right.\right.$ given by $M^{0}(x, y, t)=M(x, y, t)$ for all $x, y \in X$, $t>0$, and $M^{0}(x, y, 0)=\bigwedge_{t>0} M(x, y, t)$. Then $\left(X, M^{0}, *\right)$ is an extended fuzzy metric space if and only if $(X, M, *)$ is a fuzzy metric space satisfying for each $x, y \in X$ the condition $\bigwedge_{t>0} M(x, y, t)>0$. 
Proof. Suppose that $\left(M^{0}, *\right)$ is an extended fuzzy metric on $X$. Then, clearly, $(M, *)$ is a fuzzy metric on $X$. Now, we will see that $\bigwedge_{t>0} M(x, y, t)>0$ for all $x, y \in X$.

Take $x, y \in X$. Since $M_{x, y}$ is not decreasing on $] 0,+\infty\left[\right.$ and $M_{x, y}^{0}$ is continuous at $t=0$ then

$$
\bigwedge_{t>0} M(x, y, t)=\lim _{t \rightarrow 0} M(x, y, t)=\lim _{t \rightarrow 0} M_{x, y}^{0}(t)=M_{x, y}^{0}(0)=M^{0}(x, y, 0)>0 .
$$

Conversely, let $(X, M, *)$ be a fuzzy metric space satisfying $\bigwedge_{t>0} M(x, y, t)>$ 0 for each $x, y \in X$. Attending to the hypothesis and by construction of $M^{0}$ we have that (EFM1) and (EFM3) are fulfilled. We will show the rest of axioms.

(EFM2) Suppose $M^{0}(x, y, t)=1$ for some $t>0$. Then, $M(x, y, t)=$ 1 and so $x=y$. If $M^{0}(x, y, 0)=1$ then $\bigwedge_{t>0} M(x, y, t)=1$ and thus $M(x, y, t)=1$ for all $t>0$, and so $x=y$. Obviously, if $x=y$ then $M^{0}(x, y, t)=1$ for all $t \geq 0$.

(EFM4) Let $x, y, z \in X$. We will distinguish three possibilities on $t, s \geq 0$.

1. If $t, s>0$, then (EFM4) is fulfilled since $M$ is a fuzzy metric.

2. Suppose $t>0$ and $s=0$ (the case $t=0$ and $s>0$ is analogous). Then for $\varepsilon \in] 0, t[$ we have that

$M^{0}(x, z, t+0)=M^{0}(x, z, t)=M(x, z, t) \geq M(x, y, t-\varepsilon) * M(y, z, \varepsilon)$.

Then, taking limits as $\varepsilon$ tends to 0 in the last inequality we obtain

$$
\begin{gathered}
M^{0}(x, z, t+0) \geq \lim _{\varepsilon \rightarrow 0}(M(x, y, t-\varepsilon) * M(y, z, \varepsilon))= \\
=\left(\lim _{\varepsilon \rightarrow 0} M(x, y, t-\varepsilon)\right) *\left(\lim _{\varepsilon \rightarrow 0} M(y, z, \varepsilon)\right)=
\end{gathered}
$$




$$
=M(x, y, t) *\left(\bigwedge_{\varepsilon>0} M(y, z, \varepsilon)\right)=M^{0}(x, y, t) * M^{0}(y, z, 0) .
$$

3. Suppose $t=s=0$. Then, we have that

$$
\begin{aligned}
& M^{0}(x, z, 0+0)=M^{0}(x, z, 0)=\bigwedge_{t>0} M(x, z, t)=\lim _{t \rightarrow 0} M(x, z, t) \geq \\
\geq & \lim _{t \rightarrow 0}(M(x, y, t / 2) * M(y, z, t / 2))=\left(\lim _{t \rightarrow 0} M(x, y, t / 2)\right) *\left(\lim _{t \rightarrow 0} M(y, z, t / 2)\right)= \\
= & \left(\bigwedge_{t>0} M(x, y, t)\right) *\left(\bigwedge_{t>0} M(y, z, t)\right)=M^{0}(x, y, 0) * M^{0}(y, z, 0) .
\end{aligned}
$$

(EFM5) Since $M_{x, y}$ is continuous on $] 0,+\infty[$, and $] 0,+\infty[$ is open in $\left[0,+\infty\left[\right.\right.$, with the usual topology of $\mathbb{R}$ restricted to $\left[0,+\infty\left[\right.\right.$, then $M_{x, y}^{0}$ is continuous at each point of $] 0,+\infty[$ for each $x, y \in X$. For $t=0$ we have that

$$
\lim _{t \rightarrow 0} M^{0}(x, y, t)=\lim _{t \rightarrow 0} M(x, y, t)=\bigwedge_{t>0} M(x, y, t)=M^{0}(x, y, 0),
$$

and so $M_{x, y}^{0}$ is continuous at $t=0$.

Hence, $\left(X, M^{0}, *\right)$ is an extended fuzzy metric space.

An immediate consequence of the preceding result is that given an extended fuzzy metric space $\left(X, M^{0}, *\right)$, then $M_{x, y}^{0}:[0,+\infty[\rightarrow] 0,1]$ is a nondecreasing continuous function satisfying $M_{x, y}^{0}(0)=\bigwedge_{t>0} M^{0}(x, y, t)$, for all $x, y \in X$. Furthermore, we can deduce the following result proved by Gregori et al. in [17].

Proposition 2.2.5. Let $(X, M, *)$ be a fuzzy metric space. Define

$$
N_{M}(x, y)=\bigwedge_{t>0} M(x, y, t)
$$


Then $\left(N_{M}, *\right)$ is a stationary fuzzy metric on $X$ if and only if $\bigwedge_{t>0} M(x, y, t)>$ 0 for all $x, y \in X$.

Theorem 2.2.4 motivates the introduction of the following definition.

Definition 2.2.6. Let $(X, M, *)$ be a fuzzy metric space. $M$ is called extendable if for each $x, y \in X$ the condition $\bigwedge_{t>0} M(x, y, t)>0$ is satisfied. In such a case, we will say that $M^{0}$ is the (fuzzy metric) extension of $M$, and that $M$ is the restriction of $M^{0}$.

From now on, by $N_{M}$ we are referring to the stationary fuzzy metric defined in Proposition 2.2.5, whenever $(X, M, *)$ be an extendable fuzzy metric space. So, by the aforementioned proposition we have that $M$ is extendable if and only if $\left(N_{M}, *\right)$ is a stationary fuzzy metric on $X$. Besides, by Theorem 2.2.4 we have that $M$ is extendable if and only if $\left(X, M^{0}, *\right)$ is an extended fuzzy metric space where $M^{0}$ is given by

$$
M^{0}(x, y, t)= \begin{cases}N_{M}(x, y), & t=0, \\ M(x, y, t), & t>0 .\end{cases}
$$

We continue our study providing an example of a non-extendable fuzzy metric.

Example 2.2.7. Let $(X, d)$ be a metric space where $X$ has at least two points. Then, the standard fuzzy metric (Example 1.2.5) $\left(X, M_{d}, \wedge\right)$ is not extendable. Indeed, given $x, y \in X$ such that $x \neq y$, then $d(x, y) \neq 0$ and so

$$
\bigwedge_{t>0} M_{d}(x, y, t)=\lim _{t \rightarrow 0} \frac{t}{t+d(x, y)}=0 .
$$

Remark 2.2.8. In the following we will associate to an extendable fuzzy metric $M$ the extended fuzzy metric $M^{0}$ and the stationary fuzzy metric $N_{M}$, above defined. Then, it is satisfied $M^{0}(x, y, 0)=\bigwedge_{t>0} M(x, y, t)=N_{M}(x, y)$ 
for all $x, y \in X$. In analogous way, we will associate $M$ and $N_{M}$ to an extended fuzzy metric $M^{0}$.

Notice that due to the continuity of the real function $M_{x, y}^{0}$ on $[0,+\infty[$, whenever $M^{0}$ is an extended fuzzy metric, then the extension $M^{0}$ of an extendable fuzzy metric $M$, is unique.

\subsection{Topology deduced from an extended fuzzy metric}

In this section, we justify that we can define a topology from an extended fuzzy metric. We proceed in a similar way as in the fuzzy metric case. So, we begin defining the concepts of open and closed ball.

Definition 2.3.1. Let $\left(X, M^{0}, *\right)$ be an extended fuzzy metric space. For $x \in X, r \in] 0,1[$ and $t \geq 0$, we define the open ball of center $x$, radius $r$ and parameter $t$ as

$$
B_{M^{0}}(x, r, t)=\left\{y \in X: M^{0}(x, y, t)>1-r\right\} .
$$

In an analogous way the closed ball is

$$
B_{M^{0}}[x, r, t]=\left\{y \in X: M^{0}(x, y, t) \geq 1-r\right\} .
$$

Clearly, for $t>0$ we have that $B_{M^{0}}(x, y, t)=B_{M}(x, y, t)$ and $B_{M^{0}}[x, y, t]=$ $B_{M}[x, y, t]$. Besides, $B_{M^{0}}(x, y, 0)=B_{N_{M}}(x, y)$ and $B_{M^{0}}[x, y, 0]=B_{N_{M}}[x, y]$.

Notice that for all $x \in X, t>0, r \in] 0,1\left[\right.$ we have that $B_{M^{0}}(x, r, 0) \subset$ $B_{M}(x, r, t)$ and $B_{M^{0}}[x, r, 0] \subset B_{M}[x, r, t]$. 
Let $\left(X, M^{0}, *\right)$ be an extended fuzzy metric space. It is well known that the family $\left\{B_{N_{M}}(x, r): x \in X, r \in\right] 0,1[\}$ is a base for the topology $\tau_{N_{M}}$ on $X$ deduced from the stationary fuzzy metric $N_{M}$. So, the family given by $\left\{B_{M^{0}}(x, r, 0): x \in X, r \in\right] 0,1[\}$ is a base for the topology $\tau_{N_{M}}$ on $X$.

Definition 2.3.2. Let $(X, M, *)$ be an extended fuzzy metric space. Then, the family given by

$$
\left\{B_{M^{0}}(x, r, 0): x \in X, r \in\right] 0,1[\}
$$

is a base for a topology $\tau_{M^{0}}$ on $X$, that will be called deduced from $M^{0}$.

Clearly, the open balls $B_{M^{0}}(x, y, 0)$ are $\tau_{M^{0} \text {-open and the closed balls are }}$

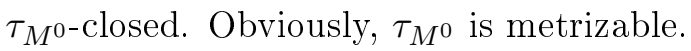

Moreover, since for all $x \in X, r \in] 0,1\left[, t>0\right.$ we have that $B_{M^{0}}(x, r, 0) \subseteq$ $B_{M}(x, r, t)$, then it is obvious that $\tau_{M^{0}} \succeq \tau_{M}$. Consequently, the open balls

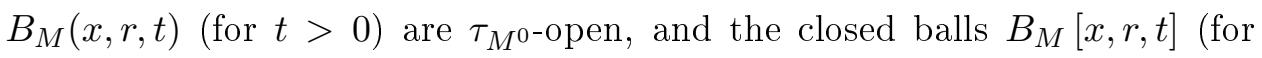
$t>0)$ are $\tau_{M^{0} \text {-closed. }}$

Now, we focus on convergence of sequences in $\tau_{M^{0}}$. We provide the next characterization for the extended fuzzy metric case (compare with Proposition 1.2.9).

Proposition 2.3.3. Let $\left(X, M^{0}, *\right)$ be an extended fuzzy metric space. A

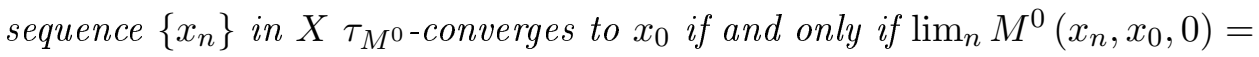
1.

Proof. Since $\tau_{N_{M}}=\tau_{M^{0}}$, then a sequence $\left\{x_{n}\right\}$ in $X \tau_{M^{0} \text {-converges to }}$ $x_{0} \in X$ if and only if $\left\{x_{n}\right\} \tau_{N_{M}}$-converges to $x_{0}$. By Proposition 1.2.9 we have that $\left\{x_{n}\right\} \tau_{N_{M}}$-converges to $x_{0}$ if and only if $\lim _{n} N_{M}\left(x_{n}, x_{0}\right)=1$. 


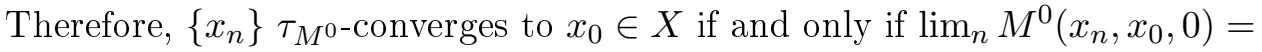
$\lim _{n} N_{M}\left(x_{n}, x_{0}\right)=1$, as we claimed.

Remark 2.3.4. On account of the exposed in this section, one can observe the similarity between extended fuzzy metrics and classical metrics, from the topological point of view. Indeed, in an extended fuzzy metric, the parameter $t$ does not play any role in the topological concepts. Nevertheless, such parameter remains being essential in different "metric" concepts for extendable fuzzy metrics, as we will see in the rest of the paper.

\subsection{Relationship between $\tau_{M^{0} \text {-convergence and }}$ $s$-convergence in extendable fuzzy metrics}

In this section, we compare $\tau_{M^{0}}$-convergent sequences with $s$-convergent ones, a stronger concept of convergence introduced in [17] (Definition 1.2.11). Such comparison is framed in the class of extendable fuzzy metrics. In order to fulfil the main goal of this section, we introduce the next proposition.

Proposition 2.4.1. Let $\left(X, M^{0}, *\right)$ be an extended fuzzy metric space. Then $M^{0}$ is continuous with respect to the product topology, where $X$ is endowed with $\tau_{M^{0}}$ and $[0,+\infty[$ with the usual topology of $\mathbb{R}$ restricted to it.

Proof. Let $\left\{\left(x_{n}, y_{n}, t_{n}\right)\right\}$ be a sequence in $X^{2} \times[0, \infty[$ which converges to $(x, y, t) \in[0, \infty[$ with respect to the product topology, where $X$ is endowed with $\tau_{M^{0}}$ and $[0,+\infty[$ with the usual topology of $\mathbb{R}$ restricted to it.

We will prove that $\lim _{n} M^{0}\left(x_{n}, y_{n}, t_{n}\right)=M^{0}(x, y, t)$ (i.e. $\left\{\left(x_{n}, y_{n}, t_{n}\right)\right\}$ converges to $M^{0}(x, y, t)$ in the usual topology of $\left.\mathbb{R}\right)$. To this end, we will distinguish two cases: 
1. Suppose that $t>0$. Without loss of generality, we can suppose that $t_{n}>0$ for all $n \in \mathbb{N}$.

Following [39, Proposition 1], if we consider the restriction $M$ of the extended fuzzy metric $M^{0}$, then $M$ is continuous on $\left.X^{2} \times\right] 0,+\infty[$ with respect to the product topology, where $X$ is endowed with $\tau_{M}$. Therefore,

$$
\lim _{n} M^{0}\left(x_{n}, y_{n}, t_{n}\right)=\lim _{n} M\left(x_{n}, y_{n}, t_{n}\right)=M(x, y, t)=M^{0}(x, y, t),
$$

and so $M^{0}$ is continuous at $(x, y, t)$.

2. Suppose now that $t=0$.

Let $\left\{\left(x_{n}, y_{n}, t_{n}\right)\right\}$ be a sequence that converges to $(x, y, 0) \in X^{2} \times\{0\}$. It follows that, for every $n \in \mathbb{N}$,

$$
M^{0}\left(x_{n}, y_{n}, t_{n}\right) \geq M^{0}\left(x_{n}, x, 0\right) * M^{0}\left(x, y, t_{n}\right) * M^{0}\left(y, y_{n}, 0\right),
$$

and

$$
M^{0}\left(x, y, t_{n}\right) \geq M^{0}\left(x, x_{n}, 0\right) * M^{0}\left(x_{n}, y_{n}, t_{n}\right) * M^{0}\left(y_{n}, y, 0\right) .
$$

If we take limits on both equations and we use Proposition 2.3.3, we obtain the following expressions.

$$
\lim _{n} M^{0}\left(x_{n}, y_{n}, t_{n}\right) \geq \lim _{n} M^{0}\left(x, y, t_{n}\right),
$$

and

$$
\lim _{n} M^{0}\left(x, y, t_{n}\right) \geq \lim _{n} M^{0}\left(x_{n}, y_{n}, t_{n}\right) .
$$

Now, by continuity of the function $M_{x, y}^{0}$ we have that $\lim _{n} M^{0}\left(x, y, t_{n}\right)=$ $M^{0}(x, y, 0)$. We deduce that $\lim _{n} M^{0}\left(x_{n}, y_{n}, t_{n}\right)=M^{0}(x, y, 0)$ and so $M^{0}$ is continuous at $(x, y, 0)$.

Hence, $M^{0}$ is continuous at each point of $X^{2} \times\{0\}$. 
The previous result is useful to prove the following proposition.

Proposition 2.4.2. Let $M$ be an extendable fuzzy metric on $X$. If $\left\{x_{n}\right\}$ is

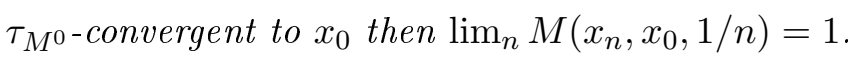

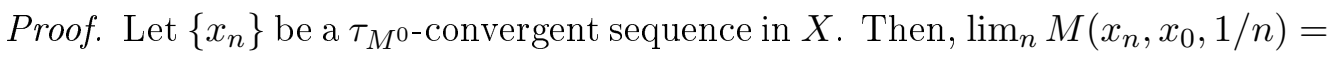
$\lim _{n} M^{0}\left(x_{n}, x_{0}, 1 / n\right)=M^{0}\left(x_{0}, x_{0}, 0\right)=1$, since $M^{0}$ is continuous and the sequence $\left\{\left(x_{n}, x_{0}, 1 / n\right)\right\}$ converges to $\left(x_{0}, x_{0}, 0\right)$ in the product topology.

The following example proves that the converse of the last proposition is false, in general.

Example 2.4.3. (see [17, Example 4.3]). Consider the extendable fuzzy metric space $(X, M, *)$, where $X=] 0,1], *$ is the Lukasievicz $t$-norm, and $M$ is given by

$$
M(x, y, t)=\left\{\begin{array}{cc}
1-\frac{1}{2} d(x, y)^{t} & 0 \leq t \leq 1 \\
1-\frac{1}{2} d(x, y) & t>1
\end{array}\right.
$$

where $d$ is the usual metric of $\mathbb{R}$. The sequence $\left\{x_{n}\right\}$, where $x_{n}=(1 / 2)-$ $\left(1 / n^{n}\right)$ for all $n \geq 2$, is $\tau_{M}$-convergent to $1 / 2$ since $\tau_{M}$ is the usual topology of $\mathbb{R}$ restricted to $] 0,1]$. Moreover, $\lim _{n} M\left(x_{n}, 1 / 2,1 / n\right)=\lim _{n}\left(1-\frac{1}{2}\left(\frac{1}{n^{n}}\right)^{1 / n}\right)=$ 1 and so $\left\{x_{n}\right\}$ is $s$-convergent. Now,

$$
N_{M}(x, y)=\bigwedge_{t>0} M(x, y, t)= \begin{cases}1 & \text { if } x=y \\ \frac{1}{2} & \text { if } x \neq y\end{cases}
$$

Therefore, $\lim _{n} M^{0}\left(x_{n}, 1 / 2,0\right)=\lim _{n} N_{M}\left(x_{n}, 1 / 2\right)=1 / 2$ and so $\left\{x_{n}\right\}$ is not

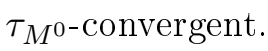

On account of the above example, an interesting question is to characterize those extendable fuzzy metric spaces in which $s$-convergent sequences 
are $\tau_{M_{0}}$-convergent. We approach this problem in the rest of the section. To such goal, we begin expressing Theorem 4.2 of [17] in our context.

Theorem 2.4.4. Let $M$ be an extendable fuzzy metric on $X$. Then, $\tau_{M}=$ $\tau_{M^{0}}$ if and only if $M$ is an s-fuzzy metric.

So, an immediate corollary of the previous theorem is the following one.

Corollary 2.4.5. Let $M$ be an extendable fuzzy metric on $X$. If $M$ is an $s$-fuzzy metric then every s-convergent sequence is $\tau_{M^{0}}$-convergent.

Even more, the next theorem shows that the converse of the preceding corollary is also true.

Theorem 2.4.6. Let $M$ be an extendable fuzzy metric on $X$. If every $s$ -

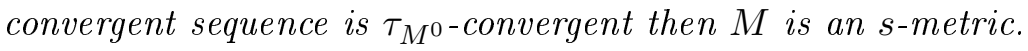

Proof. Let $M$ be an extendable fuzzy metric on $X$.

Suppose that $M$ is not an $s$-fuzzy metric. Then, there exists a $\tau_{M^{-}}$ convergent sequence $\left\{x_{n}\right\}$ to some $x_{0} \in X$, which is not $s$-convergent (to $x_{0}$ ) in $(X, M)$. We will construct an s-convergent subsequence $\left\{x_{n_{k}}\right\}$ of $\left\{x_{n}\right\}$,

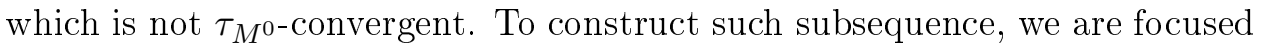
in two facts:

First, since $\left\{x_{n}\right\}$ is $\tau_{M}$-convergent then for each $\left.\varepsilon \in\right] 0,1[$ and each $t>0$ we can find $n_{0} \in \mathbb{N}$ such that $M\left(x_{n}, x_{0}, t\right)>1-\varepsilon$ whenever $n \geq n_{0}$.

Moreover, $\left\{x_{n}\right\}$ is not $s$-convergent and so it is not $\tau_{M^{0}}$-convergent. Then, by Proposition 2.3.3 we have that there exists $\left.\varepsilon_{0} \in\right] 0,1[$ such that for each $k \in \mathbb{N}$ we can find $n_{k}>k$ such that $M^{0}\left(x_{n_{k}}, x_{0}, 0\right) \leq 1-\varepsilon_{0}$.

Fix $k=2$. On the one hand, if we consider $\varepsilon=t=\frac{1}{2}$, then there exists 
$n_{2}^{\prime} \in \mathbb{N}$ such that $M\left(x_{n}, x_{0}, \frac{1}{2}\right)>1-\frac{1}{2}$ whenever $n \geq n_{2}^{\prime}$. On the other hand, given $n_{2}^{\prime} \in \mathbb{N}$ we can find $n_{2}>n_{2}^{\prime}$ such that $M^{0}\left(x_{n_{2}}, x_{0}, 0\right) \leq 1-\varepsilon_{0}$.

From this element $x_{n_{2}}$, we construct inductively on $k \in \mathbb{N}$ the announced subsequence of $\left\{x_{n}\right\}$ as follows.

Take $k \in \mathbb{N}$. As before, there exists $n_{k}^{\prime} \in \mathbb{N}$, with $n_{k}^{\prime} \geq n_{k-1}$, such that $M\left(x_{n}, x_{0}, \frac{1}{k}\right)>1-\frac{1}{k}$ whenever $n \geq n_{k}^{\prime}$. Furthermore, given $n_{k}^{\prime} \in \mathbb{N}$ we can find $n_{k}>n_{k}^{\prime}$ such that $M^{0}\left(x_{n_{k}}, x_{0}, 0\right) \leq 1-\varepsilon_{0}$.

Therefore, the constructed subsequence $\left\{x_{n_{k}}\right\}$ satisfies the following properties:

$$
M\left(x_{n_{k}}, x_{0}, 1 / k\right)>1-1 / k,
$$

and

$$
M^{0}\left(x_{n_{k}}, x_{0}, 0\right) \leq 1-\varepsilon_{0},
$$

for each $k \in \mathbb{N}$. So, taking limits in the above two inequalities we have that

$$
\lim _{k} M\left(x_{n_{k}}, x_{0}, 1 / k\right)=1
$$

and

$$
\lim _{k} M^{0}\left(x_{n_{k}}, x_{0}, 0\right) \leq 1-\varepsilon_{0} .
$$

Thus, $\left\{x_{n_{k}}\right\}$ is an $s$-convergent sequence that is not $\tau_{M^{0} \text {-convergent and the }}$ proof is concluded.

As a consequence of Corollary 2.4.5 and Theorem 2.4.6, we can state for an extendable fuzzy metric the following corollary.

Corollary 2.4.7. Let $M$ be an extendable fuzzy metric on $X$. Then, $M$ is an s-metric if and only if every s-convergent sequence is $\tau_{M^{0}}$-convergent. 


\subsection{Cauchyness and completeness}

This section is dedicated to study the completeness of extended fuzzy metric spaces. To this goal, we begin introducing a concept of Cauchy sequence in such spaces.

Definition 2.5.1. Let $\left(X, M^{0}, *\right)$ be an extended fuzzy metric space. A sequence $\left\{x_{n}\right\}$ in $X$ is called $M^{0}$-Cauchy if given $\left.\varepsilon \in\right] 0,1[$ we can find $n_{\varepsilon} \in \mathbb{N}$ such that $M^{0}\left(x_{n}, x_{m}, 0\right)>1-\varepsilon$ for all $n, m \geq n_{\varepsilon}$. Then, $\left\{x_{n}\right\}$ is $M^{0}$-Cauchy if and only if $\lim _{m, n} M^{0}\left(x_{m}, x_{n}, 0\right)=1$.

Remark 2.5.2. From now on, we will say that a sequence is $M^{0}$-convergent

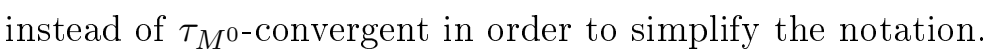

An immediate relationship between $M^{0}$-convergent sequences and $M^{0}$ Cauchy ones is the next.

Proposition 2.5.3. Let $\left(X, M^{0}, *\right)$ be an extended fuzzy metric space. Every $M^{0}$-convergent sequence is $M^{0}$-Cauchy.

Proof. Suppose $\left\{x_{n}\right\}$ is $M^{0}$-convergent to $x_{0}$. The conclusion follows from the inequality $M^{0}\left(x_{m}, x_{n}, 0\right) \geq M^{0}\left(x_{m}, x_{0}, 0\right) * M^{0}\left(x_{0}, x_{n}, 0\right)$.

The $M^{0}$-Cauchy's concept is according to the idea of Cauchy sequence in [7], since it can be expressed as $\lim _{m, n} M^{0}\left(x_{m}, x_{n}, t\right)=1$ for all $t \geq 0$. Clearly, every $M^{0}$-Cauchy sequence is $M$-Cauchy, and the converse is false, in general, as the following example shows.

Example 2.5.4. Consider the extendable fuzzy metric space $(X, M, *)$, where $X=] 0,+\infty[, *$ is the product $t$-norm and $M$ is given by

$$
M(x, y, t)=\frac{\min \{x, y\}+t}{\max \{x, y\}+t}
$$


for each $x, y \in X$ and $t>0$.

The sequence $\left\{x_{n}\right\}$, where $x_{n}=\frac{1}{n}$ for each $n \in \mathbb{N}$, is $M$-Cauchy. Indeed, for each $t>0$ we have that

$$
\lim _{n, m} M\left(x_{n}, x_{m}, t\right)=\frac{\min \{1 / n, 1 / m\}+t}{\max \{1 / n, 1 / m\}+t}=1 .
$$

Nevertheless, $\left\{x_{n}\right\}$ is not $M_{0}$-Cauchy. Indeed, observe that

$$
M^{0}(x, y, 0)=\bigwedge_{t>0} M(x, y, t)=\bigwedge_{t>0} \frac{\min \{x, y\}+t}{\max \{x, y\}+t}=\frac{\min \{x, y\}}{\max \{x, y\}},
$$

for each $x, y \in X$.

Then, if $\left\{x_{n}\right\}$ was $M^{0}$-Cauchy we will have that

$$
\lim _{n, m} M^{0}\left(x_{n}, x_{m}, 0\right)=\lim _{n, m} \frac{\min \{1 / n, 1 / m\}}{\max \{1 / n, 1 / m\}}=1 .
$$

Now, if we consider $m=2 n$, then

$$
\lim _{n, m} \frac{\min \{1 / n, 1 / m\}}{\max \{1 / n, 1 / m\}}=\lim _{n}=\frac{1 / 2 n}{1 / n}=\frac{1}{2},
$$

a contradiction.

We continue introducing the following notion of completeness in a natural way.

Definition 2.5.5. An extended fuzzy metric space $\left(X, M^{0}, *\right)$ is called complete if every $M^{0}$-Cauchy sequence is $M^{0}$-convergent. It is also said that $M^{0}$ is complete, and, if confusion does not arise, that $X$ is $M^{0}$-complete.

An immediate property of the above definition is the next one.

Proposition 2.5.6. $\left(X, M^{0}, *\right)$ is complete if and only if $\left(X, N_{M}, *\right)$ is complete. 
Proof. The sequence $\left\{x_{n}\right\}$ is $M^{0}$-Cauchy if and only if it is $N_{M^{-} \text {-Cauchy. }}$ Then, the conclusion follows from the fact that $\tau_{M^{0}}=\tau_{N_{M}}$.

The next proposition shows the relationship between $M^{0}$-completeness and $M$-completeness, when we consider an extendable $s$-fuzzy metric.

Proposition 2.5.7. Let $M$ be an extendable s-fuzzy metric on $X$. If $(X, M, *)$ is complete then $\left(X, M^{0}, *\right)$ is complete.

Proof. Let $(X, M, *)$ be a complete extendable $s$-fuzzy metric space and let $\left\{x_{n}\right\}$ be an $M^{0}$-Cauchy sequence in $X$. Then, $\left\{x_{n}\right\}$ is $M$-Cauchy and thus $\left\{x_{n}\right\}$ is $\tau_{M}$-convergent in $X$, since $M$ is complete. Now, by Theorem 2.4.4 we conclude that $\left\{x_{n}\right\}$ is $M^{0}$-convergent.

The converse of the last proposition is false, in general, as it is shown in the following example.

Example 2.5.8. Let $(X, M, *)$ be the extendable $s$-fuzzy metric space, where $X=] 0,+\infty\left[, M(x, y, t)=\frac{\min \{x, y\}+t}{\max \{x, y\}+t}\right.$ and $*$ is the $t$-norm product (see [17]).

We notice that $\tau_{M}$ is the usual topology of $\mathbb{R}$ restricted to $] 0,+\infty[$. Furthermore, $\tau_{M^{0}}=\tau_{M}$ since $M$ is an $s$-fuzzy metric.

On the one hand, we have that $\left(X, N_{M}, *\right)$ is complete (see $[16,38]$ ), where $N_{M}$ is given by

$$
N_{M}(x, y)=M^{0}(x, y, 0)=\frac{\min \{x, y\}}{\max \{x, y\}} .
$$

We conclude, by Proposition 2.5.6, that $\left(X, M^{0}, *\right)$ is complete. 
On the other hand, $(X, M, *)$ is not complete, since $\{1 / n\}$ is an $M$ Cauchy sequence in $X$, but it does not converge for $\tau_{M}$.

To finish this section we provide an example, which shows that for every metrizable topological space we can find a compatible non-stationary extendable fuzzy metric which is an $s$-fuzzy metric.

Example 2.5.9. Let $(X, \tau)$ be a metrizable topological space. Suppose that $d$ is a compatible metric on $X$, i.e. $\tau(d)=\tau$. Consider the extendable fuzzy metric $M_{\phi}(x, y, t)=\frac{\phi(t)}{\phi(t)+d(x, y)}$ of Example 2.2.3 (c). It is not hard to check that $\tau_{M_{\phi}}=\tau$.

If $\left\{x_{n}\right\}$ is $\tau_{M_{\phi}}$-convergent to $x_{0}$ then

$$
\lim _{n} M_{\phi}\left(x_{n}, x_{0}, 1 / n\right)=\lim _{n} \frac{\phi(1 / n)}{\phi(1 / n)+d\left(x_{n}, x_{0}\right)}=\frac{\phi(0)}{\phi(0)}=1 .
$$

Then $M_{\phi}$ is an extendable $s$-fuzzy metric on $X$, and thus by Theorem 2.4.4, $\tau_{M_{\phi}^{0}}=\tau_{M_{\phi}}$.

\subsection{Contractivity and fixed point theorems}

In this section we show a method for giving more general versions of fixed point theorems in extendable fuzzy metrics $M$ by means of a weaker contractive condition (Remark 2.6.5) on the extended fuzzy metric $M^{0}$. We begin recalling a notion of contractive mapping introduced by Mihet in [35]. It was adapted by Gregori and Miñana in [11] to the George and Veeramani context as follows.

Definition 2.6.1. Let $\Psi$ be the class of all mappings $\psi:] 0,1] \rightarrow] 0,1]$ such that $\psi$ is continuous, non-decreasing and $\psi(t)>t$ for all $t \in] 0,1[$. Let 
$\psi \in \Psi$. A mapping $f: X \rightarrow X$ is called fuzzy $\psi$-contractive mapping if:

$$
M(f(x), f(y), t) \geq \psi(M(x, y, t)) \text { for all } x, y \in X \text { and } t>0 .
$$

Accordingly to the above definition and extending the classical concept of contractive sequence, then a sequence $\left\{x_{n}\right\}$ in $X$ is called (fuzzy) $\psi$ contractive sequence if it satisfies

$$
M\left(x_{n+1}, x_{n+2}, t\right) \geq \psi\left(M\left(x_{n}, x_{n+1}, t\right)\right) \text { for all } n \in \mathbb{N} \text { and } t>0 .
$$

An immediate consequence of the previous notion is the next proposition.

Proposition 2.6.2. Let $(X, M, *)$ be a fuzzy metric space. If $f: X \rightarrow X$ is $\psi$-contractive then, for each $x_{0} \in X$, the sequence of iterates $\left\{x_{n}\right\}$ where $x_{1}=f\left(x_{0}\right), \ldots, x_{n}=f\left(x_{n-1}\right)$, for $n=2,3, \cdots$, is $\psi$-contractive.

Proof. It is straightforward.

We recall the concept of contractivity given by Gregori and Sapena in [25].

Definition 2.6.3. Let $(X, M, *)$ be a fuzzy metric space. A mapping $f$ : $X \rightarrow X$ is called fuzzy contractive if there exists $k \in] 0,1[$ such that

$$
\frac{1}{M(f(x), f(y), t)}-1 \leq k\left(\frac{1}{M(x, y, t)}-1\right)
$$

for each $x, y \in X$ and $t>0$. ( $k$ is called the contractive constant of $f$.)

Mihet observed in [35] that a fuzzy contractive mapping is a fuzzy $\psi$ contractive one for $\psi(s)=\frac{s}{s+k(1-s)}$ for each $\left.\left.s \in\right] 0,1\right]$.

As we have proceeded throughout the paper, we will adapt the notion of fuzzy $\psi$-contractive mapping to extended fuzzy metrics. 
Definition 2.6.4. Let $(X, M, *)$ be an extendable fuzzy metric space. A mapping $f: X \rightarrow X$ is called fuzzy $\psi$ - $M^{0}$-contractive if Equation (2.1) is satisfied for all $t \geq 0$. Particularly, $f$ is called fuzzy $\psi$-0-contractive if Equation (2.1) is satisfied for $t=0$.

Remark 2.6.5. Due to the continuity of $M_{x, y}^{0}$ for all $x, y \in X$, it is clear that $f$ is $\psi$ - $M^{0}$-contractive if and only if $f$ is $\psi$-contractive. Nevertheless, the next example shows that the fuzzy $\psi$-0-contractive condition is weaker than the fuzzy $\psi$ - $M$-contractive one.

Example 2.6.6. Consider $X=\mathbb{R}$ endowed with the usual metric $d$. Define the fuzzy set $M$ on $\left.X^{2} \times\right] 0,+\infty[$ as follows

$$
M(x, y, t)=\frac{1+\alpha(t) d(x, y)}{1+d(x, y)}
$$

where $\alpha:\left[0,+\infty\left[\rightarrow\left[0,1\left[\right.\right.\right.\right.$ is a function given by $\alpha(t)=\frac{t}{1+t}$ for all $t \geq 0$. We will see that $(X, M, *)$ is a fuzzy metric for the Lukasievicz $t$-norm $* \mathcal{L}$.

Clearly, (GV1)-(GV3) and (GV5) are satisfied. We will only prove the triangle inequality (GV4). First, note that

$M(x, y, t)=\frac{1+\alpha(t) d(x, y)}{1+d(x, y)}=1-\frac{(1-\alpha(t)) d(x, y)}{1+d(x, y)}=1-\frac{d(x, y)}{(1+t)(1+d(x, y))}$.

We will prove that $M(x, z, t+s) \geq M(x, y, t) *_{\mathcal{L}} M(y, z, s)$, when the right side is positive.

Therefore, we must prove that

$1-\frac{d(x, z)}{(1+t+s)(1+d(x, z))} \geq 1-\frac{d(x, y)}{(1+t)(1+d(x, y))}-\frac{d(y, z)}{(1+s)(1+d(y, z))}$,

i.e. that

$$
\frac{d(x, z)}{(1+t+s)(1+d(x, z))} \leq \frac{d(x, y)}{(1+t)(1+d(x, y))}+\frac{d(y, z)}{(1+s)(1+d(y, z))} .
$$


Indeed, it is easy to verify that if $a \geq b \geq 0$ then $\frac{a}{a+1} \geq \frac{b}{b+1}$. Then, since $d(x, z) \leq d(x, y)+d(y, z)$ we have that

$$
\begin{aligned}
\frac{d(x, z)}{(1+t+s)(1+d(x, z)} & \leq \frac{d(x, y)+d(y, z)}{(1+t+s)(1+d(x, y)+d(y, z))} \\
& \leq \frac{d(x, y)+d(y, z)}{(1+\max \{t, s\})(1+\max \{d(x, y), d(y, z)\})} \\
& \leq \frac{d(x, y)}{(1+t)(1+d(x, y))}+\frac{d(y, z)}{(1+s)(1+d(y, z))}
\end{aligned}
$$

Hence $\left(M, *_{\mathcal{L}}\right)$ is a fuzzy metric on $X$, and, obviously, $M$ is extendable. Now we will see that $f: \mathbb{R} \rightarrow \mathbb{R}$ given by $f(x)=x / 2$, for $x \in \mathbb{R}$ is fuzzy $\psi$-0-contractive.

Let $x, y \in X$. Then

$$
\begin{gathered}
\frac{1}{M(f(x), f(y), 0)}-1=\left(\frac{1}{1+1 / 2|x-y|}\right)^{-1}-1=\frac{|x-y|}{2}=\frac{1}{2}(1+|x-y|-1)= \\
=\frac{1}{2}\left(\left(\frac{1}{1+|x-y|}\right)^{-1}-1\right)=\frac{1}{2}\left(\frac{1}{M(x, y, 0)}-1\right) .
\end{gathered}
$$

Then, attending to the above comment, $f$ is fuzzy $\psi$-0-contractive, for $\psi(s)=$ $\frac{s}{s+k(1-s)}$ for each $\left.\left.s \in\right] 0,1\right]$.

Now we will see that $f$ is not fuzzy contractive. Indeed, if we take $t=1$, then, for $x, y \in \mathbb{R}$ we have

$$
\frac{1}{M(f(x), f(y), 1)}-1=\left(\frac{1+\frac{1}{2} \cdot \frac{1}{2}|x-y|}{1+\frac{1}{2}|x-y|}\right)^{-1}-1=\frac{|x-y|}{4+|x-y|} .
$$

On the other hand,

$$
k\left(\frac{1}{M(x, y, 1)}-1\right)=k\left(\left(\frac{1+\frac{1}{2}|x-y|}{1+|x-y|}\right)^{-1}-1\right)=k\left(\frac{|x-y|}{2+|x-y|}\right) .
$$


In this case, the inequality

$$
\frac{1}{M(f(x), f(y), 1)}-1 \leq k\left(\frac{1}{M(x, y, 1)}-1\right)
$$

is not possible for $k \in[0,1$. Indeed, for a fixed $y \in \mathbb{R}$, if $x$ tends to $+\infty$ then we obtain $1 \leq k$, a contradiction.

According to Definition 2.6.4, we introduce the notion of fuzzy $\psi-0$ contractive sequence.

Definition 2.6.7. Let $(X, M, *)$ be an extendable fuzzy metric space. A sequence $\left\{x_{n}\right\}$ of $X$ is called fuzzy $\psi$-0-contractive if there exists $\psi \in \Psi$ such that

$$
M^{0}\left(x_{n+1}, x_{n+2}, 0\right) \geq \psi\left(M^{0}\left(x_{n}, x_{n+1}, 0\right)\right) \quad \text { for all } n \in \mathbb{N} \text {. }
$$

The sequence of iterates constructed from a fuzzy $\psi$-0-contractive mapping satisfies the following stronger property.

Proposition 2.6.8. Let $(X, M, *)$ be an extendable fuzzy metric space, and let $f: X \rightarrow X$ be a fuzzy $\psi$-0-contractive mapping. Consider, for $x_{0} \in X$, the sequence $\left\{x_{n}\right\}$ of iterates $x_{1}=f\left(x_{0}\right), x_{n}=f\left(x_{n-1}\right), n=2,3, \ldots$ Then,

$$
M^{0}\left(x_{n+1}, x_{m+1}, 0\right) \geq \psi\left(M^{0}\left(x_{n}, x_{m}, 0\right)\right.
$$

for every $n, m \in \mathbb{N}$.

Proof. It follows from the definition of fuzzy $\psi$-0-contractive function.

Mimicking the proof of Lemma 3.2 in [12] and using the preceding proposition, we obtain the following result. 
Proposition 2.6.9. Let $\left(X, M^{0}, *\right)$ be an extended fuzzy metric space and let $f: X \rightarrow X$ be a fuzzy $\psi$-0-contractive mapping. Then for each $x_{0} \in X$, the sequence of iterates $\left\{x_{n}\right\}$ is $M^{0}$-Cauchy.

As a consequence of the above result and following the arguments of [12], for instance, Corollary 3.9 of [12] admits the following more general version (Remark 2.6.5).

Theorem 2.6.10. Let $(X, M, *)$ be an extendable complete fuzzy metric space. Then, every fuzzy $\psi$-0-contractive mapping $f: X \rightarrow X$ admits a unique fixed point. 


\section{Chapter 3}

\section{Contractive sequences in $G V$-fuzzy metric spaces}

\subsection{Introduction}

Recall that in classical fixed point theory, a contractive sequence of iterates $\left\{f^{(n)}\left(x_{0}\right)\right\}$ of a self-contractive mapping $f$ on a complete metric space $X$ is constructed, for all $x_{0} \in X$. This sequence converges in $X$ since contractive sequences are Cauchy. But, what about this statement in fuzzy setting? We notice that in [25] the authors introduced a concept of fuzzy contractive sequence and they posed the following question: Is every fuzzy contractive sequence a Cauchy sequence? So far, there is no answer to this question (D. Mihet [35] gave a negative answer, but for fuzzy metrics in the sense of Kramosil and Michalek). The purpose of this chapter is to make a new contribution to this field and, at the same time, to correct an error appeared in [12]. For it, we will introduce and study a concept of strictly fuzzy contractive 
sequence.

Regarding the last paragraph, on the one hand, in our short (preliminary) Section 3.2 we notice that there are several concepts of Cauchy sequence in the literature [18]. Here we focus our attention in the two concepts used in fuzzy fixed point theory. The first one was given by M. Grabiec in [9] and it will be denoted by $G$-Cauchy (Definition 3.2.1). The second one will be called, simply, Cauchy (Definition 1.2.10) and it is due to George and Veeramani [7] (although it comes from PM-spaces [44]). It is well known that Cauchy implies $G$-Cauchy. On the other hand, with respect to (fuzzy) contractive mappings, we deal with four related concepts, named within brackets, due (chronologically) to Gregori and Sapena (GS) [25], Mihet ( $\psi$ contractive) [35], Romaguera and Tirado (RT) [40], and Wardowski $(\mathcal{H})$ [49] (Definition 3.2.2). The relationship among these concepts is shown in the following chain of (strict) implications:

RT-contractive $\Longrightarrow$ GS-contractive $\Longrightarrow \mathcal{H}$-contractive $\Longrightarrow \psi$-contractive

According to these concepts, and in a similar way to classical metrics, we obtain their corresponding concepts of (fuzzy) contractive sequence (Definition 3.2.3), which preserve the aforementioned chain of implications.

In Section 3.3 we study the relationship among these contractivity conditions and Cauchyness. Our main result is Example 3.3.13 in which we construct a $\psi$-contractive sequence in a stationary fuzzy metric, which is not Cauchy. This example points out that the concept of $\psi$-contractivity needs to be strengthened, to some strictly fuzzy contractivity, to get Cauchyness. But in what form should it be done? Our decision is based on (the proof of) Lemma 3.2 of [12], which asserts: "A $\psi$-contractive sequence $\left\{x_{n}\right\}$ satisfying $\bigwedge_{t>0} M\left(x_{1}, x_{2}, t\right)>0$, is a Cauchy sequence". It is clear, at the light of Example 3.3.13, that this lemma is false. The error in the men- 
tioned proof is due to the fact that the authors have improperly used the property: $M\left(x_{m+1}, x_{n+1}, t\right) \geq \psi\left(M\left(x_{m}, x_{n}, t\right)\right)$, for all $m, n \in \mathbb{N}$. Therefore we just define a strictly fuzzy $\psi$-contractive sequence (Definition 3.3.2) as a $\psi$-contractive sequence that satisfies that property. In this manner, the mentioned Lemma 3.2 is valid for strictly fuzzy $\psi$-contractive sequences defined in a fuzzy metric space satisfying $\bigwedge_{t>0} M(x, y, t)>0$ for every $x, y \in X$. The concept of strictly contractive sequence for a metric space is also given, in a similar way, in Definition 3.3.6. In a similar way, the other three concepts of strictly fuzzy contractive sequence are defined, and among these four concepts, again the above chain of implications is satisfied. The given concept of strictly fuzzy contractive sequence can be considered an appropriate concept, not only because it makes true Lemma 3.2 aforementioned, but also because for $x_{0} \in X$ the sequence of iterates $\left\{f^{(n)}\left(x_{0}\right)\right\}$ of a contractive mapping $f$, for each one of the mentioned contractive conditions, is strictly fuzzy contractive (Proposition 3.3.5). The reader can find another favorable argument to this new concept in Proposition 3.3.7. Example 3.3.9.(a) provides a contractive sequence which is not a strictly contractive sequence in $(\mathbb{R},|\cdot|)$. Then, using this example and Proposition 3.3.8 we construct in (b) of Example 3.3.9 a non strictly $R T$-contractive sequence. As a positive result, before our mentioned Example 3.3.13, we prove that every $\psi$-contractive sequence is $G$-Cauchy (Proposition 3.3.12).

Section 3.4 is, basically, a correction to Lemma 3.2 of [12]. Then, after properly correcting Corollary 3.8 and Lemma 3.12 of [12] we show two large classes of fuzzy metric spaces where the condition of strictly fuzzy contractivity for a sequence implies Cauchy. The section is finished with Question 3.4.4; Is every strictly fuzzy $\psi$-contractive sequence a Cauchy sequence? 


\subsection{Preliminaries}

In order to obtain fixed point theorems in fuzzy metric spaces, M. Grabiec introduced the first concept of Cauchy sequence, as follows.

Definition 3.2.1. (Grabiec [9]). A sequence $\left\{x_{n}\right\}$ in a fuzzy metric $(X, M, *)$ is called G-Cauchy if $\lim _{n} M\left(x_{n}, x_{n+p}, t\right)=1$ for each $t>0$ and each $p \in \mathbb{N}$.

In Chapter 2 we have mentioned two notions of contractive mapping in fuzzy metric spaces due to Mihet (Definition 3.3.2) and Gregori and Sapena (Definition 2.6.3). In [40] Romaguera and Tirado introduced another concept of contractive mapping. In addition, in [49] Wardowski introduced a new concept of contractive mapping using a family $\mathcal{H}$ of mappings $\eta:] 0,1] \rightarrow$ $[0,+\infty[$ satisfying that $\eta$ is onto and strictly decreasing. In order to make a self-contained chapter, we summarize these four concepts in the following definition.

Definition 3.2.2. Let $M$ be a fuzzy metric on $X$. A mapping $f: X \rightarrow X$ is called

(i) $R T$-contractive [40] if there exists $k \in] 0,1[$ such that:

$$
M(f(x), f(y), t) \geq 1-k+k M(x, y, t) \quad \text { for all } x, y \in X \text { and } t>0 .
$$

(ii) $G S$-contractive [25] if there exists $k \in] 0,1[$ such that:

$$
\frac{1}{M(f(x), f(y), t)}-1 \leq k\left(\frac{1}{M(x, y, t)}-1\right) \quad \text { for all } x, y \in X \text { and } t>0 .
$$

(iii) fuzzy $\mathcal{H}$-contractive [49] with respect to $\eta \in \mathcal{H}$ if there exists $k \in] 0,1[$ such that:

$$
\eta(M(f(x), f(y), t)) \leq k \eta(M(x, y, t)) \quad \text { for all } x, y \in X \text { and } t>0 .
$$


(iv) fuzzy $\psi$-contractive [35], with respect to $\psi \in \Psi$, if:

$$
M(f(x), f(y), t) \geq \psi(M(x, y, t)) \text { for all } x, y \in X \text { and } t>0 .
$$

The following definition is according to Definition 3.2.2.

Definition 3.2.3. Let $(X, M, *)$ be a fuzzy metric space. A sequence $\left\{x_{n}\right\}$ in $X$ is called

(i) $R T$-contractive [40] if there exists $k \in] 0,1[$ such that

$$
M\left(x_{n+1}, x_{n+2}, t\right) \geq 1-k+k M\left(x_{n}, x_{n+1}, t\right) \text { for all } n \in \mathbb{N} \text { and } t>0 .
$$

(ii) $G S$-contractive [25] if there exists $k \in] 0,1[$ such that:

$$
\frac{1}{M\left(x_{n+1}, x_{n+2}, t\right)}-1 \leq k\left(\frac{1}{M\left(x_{n}, x_{n+1}, t\right)}-1\right) \quad \text { for all } n \in \mathbb{N} \text { and } t>0 .
$$

(iii) fuzzy $\mathcal{H}$-contractive [49] with respect to $\eta \in \mathcal{H}$ if there exists $k \in] 0,1[$ satisfying

$$
\eta\left(M\left(x_{n+1}, x_{n+2}, t\right)\right) \leq k \eta\left(M\left(x_{n}, x_{n+1}, t\right)\right) \text { for all } n \in \mathbb{N} \text { and } t>0 .
$$

(iv) fuzzy $\psi$-contractive [35], with respect to $\psi \in \Psi$, if

$$
M\left(x_{n+1}, x_{n+2}, t\right) \geq \psi\left(M\left(x_{n}, x_{n+1}, t\right)\right) \text { for all } n \in \mathbb{N} \text { and } t>0 .
$$

\subsection{Strictly fuzzy contractive sequences}

In this section $(X, M, *)$ is a fuzzy metric space. By a (fuzzy) contractive sequence we refer to any of the ones mentioned in Definition 3.2.3, when specification is not needed. The following proposition shows the relationship among the different notions of contractive sequence. 
Proposition 3.3.1. The following chain of implications related to sequences, is satisfied:

RT-contractive $\Longrightarrow$ GS-contractive $\Longrightarrow \mathcal{H}$-contractive $\Longrightarrow \psi$-contractive

Proof. The first implication is obtained with a simple computation (with the same constant $k$ ). The second one is a consequence of [49, Example 3.1]. The last one is a consequence of [12, Proposition 6].

We introduce now the following concept as a result of the discussion made in Introduction.

Definition 3.3.2. We will say that a sequence $\left\{x_{n}\right\}$ is strictly fuzzy $\psi$ contractive if $M\left(x_{m+1}, x_{n+1}, t\right) \geq \psi\left(M\left(x_{m}, x_{n}, t\right)\right)$ for all $n, m \in \mathbb{N}$ and $t>0$. In an analogous way are defined the concepts of strictly fuzzy contractivity for the other three concepts of contractive sequence.

Proposition 3.3.3. A sequence $\left\{x_{n}\right\}$ is strictly fuzzy $\psi$-contractive if and only if for each $p \in \mathbb{N}, M\left(x_{m+p}, x_{n+p}, t\right) \geq \psi\left(M\left(x_{m}, x_{n}, t\right)\right)$ holds for all $n, m \in \mathbb{N}$.

Proof. Suppose that the sequence $\left\{x_{n}\right\}$ is strictly fuzzy $\psi$-contractive and let $p \in \mathbb{N}$. Take $t>0$. With an induction process on $p$, we have for all $m, n \in \mathbb{N}$ that

$$
\begin{aligned}
M\left(x_{m+p}, x_{n+p}, t\right) & \geq \psi\left(M\left(x_{m+p-1}, x_{n+p-1}, t\right)\right) \geq \ldots \geq \psi\left(\psi^{p-1)}\left(M\left(x_{m}, x_{n}, t\right)\right)\right) \\
& \geq \psi\left(M\left(x_{m}, x_{n}, t\right)\right) .
\end{aligned}
$$

The converse is obvious.

Remark 3.3.4. Analogous statements can be given for the rest of the notions of strictly fuzzy contractivity. 
The following proposition ensures the existence of strictly fuzzy contractive sequences.

Proposition 3.3.5. Let $f: X \rightarrow X$ be a contractive mapping in any of the senses in Definition 3.2.2. Then, for each $x_{0} \in X$, the sequence of iterates $\left\{f^{(n)}(x)\right\}$ is strictly fuzzy contractive for the same sense.

Proof. Let $x_{0} \in X$ and suppose that $f$ is $\psi$-contractive. Put $x_{n}=f^{(n)}(x)$, $n=1,2, \ldots$. For $t>0$ we have that

$$
M\left(x_{m+1}, x_{n+1}, t\right)=M\left(f\left(x_{m}\right), f\left(x_{n}\right), t\right) \geq \psi\left(M\left(x_{m}, x_{n}, t\right)\right)
$$

for all $m, n \in \mathbb{N}$.

The proof for the other contractive concepts is analogous.

At the light of Proposition 3.3.1 the reader can easily obtain the same chain of implications with strictly fuzzy contractive sequences instead of the contractive ones.

The concept of strictly contractive sequence can also be given in metric spaces, as follows.

Definition 3.3.6. Let $(X, d)$ be a metric space. A sequence $\left\{x_{n}\right\}$ in $(X, d)$ is strictly contractive if there exists $k \in] 0,1[$ such that

$$
d\left(x_{m+1}, x_{n+1}\right) \leq k d\left(x_{m}, x_{n}\right) \text {, for all } m, n \in \mathbb{N} .
$$

It is also said that $\left\{x_{n}\right\}$ is strictly $d$-contractive. This terminology is commonly used in topology and we will use it in other concepts without explicit mention. Obviously, if $f$ is a contractive self-mapping of $(X, d)$ then $\left\{f^{n}(x)\right\}_{n}$ is strictly $d$-contractive, for each $x \in X$. 
Proposition 3.3.7. Let $\left(\mathbb{R}, M_{d}, \cdot\right)$ be the standard fuzzy metric where $d$ is the usual Euclidean metric on $\mathbb{R}$. Then, every monotone (non decreasing or non increasing) GS-contractive sequence in $\mathbb{R}$ is strictly GS-contractive.

Proof. Suppose that $\left\{x_{n}\right\}$ is a non-decreasing $G S$-contractive sequence in $\mathbb{R}$. On account of [25, Proposition 3.9], $\left\{x_{n}\right\}$ is a $d$-contractive sequence for the same constant of contractivity, say $k \in] 0,1\left[\right.$. We will prove that $\left\{x_{n}\right\}$ is strictly $d$-contractive. Indeed, for $m, n \in \mathbb{N}$, with $m>n$, we have that

$$
d\left(x_{m+1}, x_{n+1}\right) \leq d\left(x_{m+1}, x_{m}\right)+d\left(x_{m}, x_{m-1}\right)+\ldots+d\left(x_{n+2}, x_{n+1}\right) .
$$

Since $\left\{x_{n}\right\}$ is $d$-contractive and monotone we have that

$$
\begin{aligned}
d\left(x_{m+1}, x_{n+1}\right) & \leq k d\left(x_{m}, x_{m-1}\right)+k d\left(x_{m-1}, x_{m-2}\right)+\ldots+k d\left(x_{n+1}, x_{n}\right) \\
& =k\left(d\left(x_{m}, x_{m-1}\right)+\ldots+d\left(x_{n+1}, x_{n}\right)\right) \\
& =k\left(d\left(x_{m}, x_{n}\right)\right)
\end{aligned}
$$

and so $\left\{x_{n}\right\}$ is strictly $d$-contractive. Therefore,

$$
\begin{aligned}
\frac{1}{M_{d}\left(x_{m+1}, x_{n+1}, t\right)}-1 & =\frac{t+d\left(x_{m+1}, x_{n+1}\right)}{t}-1=\frac{d\left(x_{m+1}, x_{n+1}\right)}{t} \\
& \leq \frac{k d\left(x_{m}, x_{n}\right)}{t}=k\left(\frac{1}{M_{d}\left(x_{m}, x_{n}, t\right)}-1\right)
\end{aligned}
$$

and hence $\left\{x_{n}\right\}$ is strictly $G S$-contractive.

In Example 3.3.9 we will construct a contractive sequence in $\mathbb{R}$, provided with its usual metric, which is not strictly contractive. Then, based on this sequence, we will give an example of an $R T$-contractive sequence (the strongest concept of contractivity, here considered) which is not strictly $R T$ contractive. Before, we need the following proposition. 
Proposition 3.3.8. Let $(X, d)$ be a metric space such that $d(x, y)<1$ for all $x, y \in X$. Denote by $(X, N, \mathfrak{L})$ the stationary fuzzy metric space where $N(x, y)=1-d(x, y)$. Then

(i) $\tau_{N}=\tau(d)$.

(ii) $\left\{x_{n}\right\}$ is (strictly) RT-contractive in $(X, N, \mathfrak{L})$ if and only if $\left\{x_{n}\right\}$ is (strictly) d-contractive.

(iii) $\left\{x_{n}\right\}$ is $N$-Cauchy if and only if $\left\{x_{n}\right\}$ is d-Cauchy.

(iv) $(X, N, \mathfrak{L})$ is complete if and only if $(X, d)$ is complete.

Proof. It is straightforward.

It is left to the reader to introduce a similar proposition for $\left(X, M_{d}, \cdot\right)$.

Example 3.3.9. (a) (A non-strictly contractive sequence). Consider the metric space $(X, d)$, where $X=\mathbb{R}$ and $d$ is the Euclidean metric. Let $a, b \in \mathbb{R}$ with $0 \leq a<b$. We define the sequence $\left\{x_{n}\right\}$ in $\mathbb{R}$, by recurrence as follows.

$$
\begin{gathered}
x_{1}=\frac{a+b}{2}, x_{2}=\frac{a+x_{1}}{2}, \\
x_{2 k-1}=\frac{x_{2 k-2}+x_{2 k-3}}{2}, \quad x_{2 k}=\frac{x_{2 k-1}+x_{2 k-3}}{2}, \text { for } k \geq 2 .
\end{gathered}
$$

The sequence $\left\{x_{n}\right\}$ is $d$-contractive. Indeed,

$d\left(x_{k+1}, x_{k}\right)=\left|x_{k+1}-x_{k}\right|=\left|x_{k+1}+x_{k-1}-x_{k-1}-x_{k}\right|=2\left|x_{k+2}-x_{k+1}\right|$, that is, $d\left(x_{k+2}, x_{k+1}\right) \leq \frac{1}{2} d\left(x_{k+1}, x_{k}\right)$, and so $\left\{x_{n}\right\}$ is $d$-contractive. This sequence is not strictly $d$-contractive. Indeed, for $k \geq 1$, we have 
that

$$
\begin{aligned}
d\left(x_{4 k+2}, x_{4 k}\right) & =\left|\frac{x_{4 k+1}}{2}+\frac{x_{4 k-1}}{2}-x_{4 k}\right|=\left|\frac{x_{4 k}+x_{4 k-1}}{4}+\frac{x_{4 k-1}}{2}+x_{4 k}\right| \\
& =\frac{3}{4}\left|x_{4 k-1}-x_{4 k}\right|>\frac{1}{2}\left|x_{4 k-1}-x_{4 k}\right|=\left|x_{4 k-1}-\frac{x_{4 k}-x_{4 k-1}}{2}\right| \\
& =\left|x_{4 k-1}-x_{4 k+1}\right|=d\left(x_{4 k+1}, x_{4 k-1}\right),
\end{aligned}
$$

and hence, the sequence $\left\{x_{n}\right\}$ is not strictly contractive.

On the other hand, the sequence $\left\{x_{n}\right\}$ is convergent in $\mathbb{R}$, since every contractive sequence in a metric space is Cauchy, and $\mathbb{R}$ is complete.

(b) (A non-strictly $R T$-contractive sequence). Now, take $a=0, b=1 / 2$ in the part $(a)$ of this example. The corresponding sequence $\left\{x_{n}\right\}$, named now $\left\{y_{n}\right\}$, is a non-strictly contractive sequence in the complete metric space $([0,1 / 2], d)$ and by (ii) of Proposition 3.3.8, $\left\{y_{n}\right\}$ is a non-strictly $R T$-contractive sequence in $([0,1 / 2], N, \mathfrak{L})$. Moreover, by $(i i i)-(i v)$ of Proposition 3.3.8 the sequence $\left\{y_{n}\right\}$ is $N$-Cauchy and then it converges in $[0,1 / 2]$.

Remark 3.3.10. After a tedious computation it can be proved that the sequence $\left\{x_{n}\right\}$ in (a) of Example 3.3 .9 converges to $(3 a+2 b) / 5$. Indeed, by induction on $\mathrm{k}$, it is easy to prove that

$$
x_{4 k}-x_{4 k-1}=\frac{(b-a)}{16^{k}} .
$$

Furthermore, for every $k \in \mathbb{N}$, we have that

$$
\begin{aligned}
x_{4 k} & =\frac{x_{4 k-1}+x_{4 k-3}}{2}=\frac{\left(x_{4 k-2}+x_{4 k-3}\right)+\left(x_{4 k-4}+x_{4 k-5}\right)}{4} \\
& =\frac{x_{4 k-3}+x_{4 k-5}+x_{4 k-4}+x_{4 k-5}+2 x_{4 k-4}+2 x_{4 k-5}}{8} \\
& =\frac{x_{4 k-4}+x_{4 k-5}+2 x_{4 k-5}+2 x_{4 k-4}+2 x_{4 k-5}+4 x_{4 k-4}+4 x_{4 k-5}}{16} \\
& =\frac{7 x_{4 k-4}+9 x_{4 k-5}}{16}=\frac{7 x_{4(k-1)}+9 x_{4(k-1)-1}}{16} .
\end{aligned}
$$


Now, using Equation (3.1), we have that

$$
x_{4 k}=\frac{7 x_{4(k-1)}+9\left(x_{4(k-1)}-\frac{(b-a)}{16^{k-1}}\right)}{16}=x_{4(k-1)}-\frac{9(b-a)}{16} \frac{1}{16^{k-1}} .
$$

Using the notation $y_{k}=x_{4 k}$, we get a recurrence sequence given by

$$
y_{k}=y_{k-1}-\frac{9(b-a)}{16^{k}}, \quad \text { for } k=2,3 \ldots
$$

Now, one can prove that $y_{1}=x_{4}=\frac{9 a+7 b}{16}$, and then, by a few calculations one obtains

$$
y_{k}=\frac{3 a+2 b}{5}-\frac{3(a-b)}{5} \frac{1}{16^{k}} .
$$

Therefore the sequence $\left\{y_{n}\right\}$, obviously, converges to $(3 a+2 b) / 5$. Our conclusion holds by the fact that all subsequences of a convergent sequence converge to the same point.

The following example shows another non-strictly contractive sequence.

Example 3.3.11. Let $a \in \mathbb{R}$ and let. Consider the metric space $([-a, a], d)$ where $d$ is the Euclidean metric. We will define a sequence $\left\{x_{n}\right\}$ that is convergent to zero:

$$
x_{1}=0.5 a ; \quad x_{2}=-0.8 a ; \quad x_{3}=0.3 a ; \quad x_{4}=-0.3 a .
$$

It is obvious that $d\left(x_{1}, x_{3}\right)=0.2 a<0.5 a=d\left(x_{2}, x_{4}\right)$. Let us define $\left\{x_{4 k+1}, x_{4 k+2}, x_{4 k+3}, x_{4 k+4}\right\}$ for each $k \in \mathbb{N}$ inductively by mimicking the same process with interval $\left[x_{4 k}, x_{4 k-1}\right]=\left[-0.3^{k} a, 0.3^{k} a\right]$. Clearly, this sequence is convergent to zero, but it is not strictly contractive as $d\left(x_{4 k+1}, x_{4 k+3}\right)<$ $d\left(x_{4 k+2}, x_{4 k+4}\right)$.

Now we are interested in the relationship between fuzzy contractive sequences and Cauchy sequences. The most general result that we can give is 
the following proposition, which states that every fuzzy contractive sequence of Definition 3.2.3 is G-Cauchy.

Proposition 3.3.12. Every $\psi$-contractive sequence is G-Cauchy.

Proof. Let $\left\{x_{n}\right\}$ be a $\psi$-contractive sequence. Then, for $t>0$ we have that

$$
M\left(x_{n}, x_{n+1}, t\right) \geq \psi\left(M\left(x_{n-1}, x_{n}, t\right)\right) \geq \ldots \geq \psi^{n)}\left(M\left(x_{1}, x_{2}, t\right)\right)
$$

for all $t>0$. Now for each $s \in] 0,1\left[\right.$ it is easy to verify that $\lim _{n} \psi^{n)}(s)=1$, and then $\lim _{n} M\left(x_{n}, x_{n+1}, t\right)=1$ for each $t>0$.

In the following example we show a $\psi$-contractive sequence which is not Cauchy.

Example 3.3.13 (A $\psi$-contractive sequence which is not Cauchy). Consider the real line $\mathbb{R}$ endowed with the usual Euclidean metric $d$. We define the mapping $\tilde{\psi}:[0,1] \rightarrow[0,1]$ as follows.

Consider the partition $\{[\ell /(\ell+1),(\ell+1) /(\ell+2)[: \ell=0,1,2, \ldots\}$ of $[0,1[$. We define

$$
\tilde{\psi}(s)=\left\{\begin{array}{cc}
\frac{\ell+1}{\ell+3} \cdot s+\frac{2 \ell+3}{(\ell+2)(\ell+3)}, & \text { for } s \in\left[\frac{\ell}{\ell+1}, \frac{\ell+1}{\ell+2}[,\right. \\
1 & \text { for } s=1 .
\end{array}\right.
$$

Let $\psi$ the restriction of $\tilde{\psi}$ to $] 0,1]$. Then, it is easy to verify that $\psi(s)>s$ for all $s \in] 0,1[$, and that $\psi$ is non-decreasing on $[0,1]$. Also $\psi$ is continuous on $] 0,1\left[\right.$. We will see that $\psi$ is continuous at $s=1$. Suppose that $\left\{s_{j}\right\}$ is a sequence in $[0,1]$ that converges to 1 . We will see that $\left\{\psi\left(s_{j}\right)\right\}$ converges to 1. 
Let $0<\varepsilon<1$. Choose $\ell_{0} \in \mathbb{N}$ such that $\ell_{0} /\left(\ell_{0}+3\right)>1-\varepsilon$. Then

$\psi\left(\frac{\ell_{0}}{\ell_{0}+1}\right)=\frac{\ell_{0}+1}{\ell_{0}+3} \cdot \frac{\ell_{0}}{\ell_{0}+1}+\frac{2 \ell_{0}+2}{(\ell+2)(\ell+3)}>\frac{\ell_{0}+1}{\ell_{0}+3} \cdot \frac{\ell_{0}}{\ell_{0}+1}=\frac{\ell_{0}}{\ell_{0}+3}>1-\varepsilon$

Take $\delta>0$ such that $\ell_{0} /\left(\ell_{0}+1\right)<\delta<1$. Then, there exists $j_{0} \in \mathbb{N}$ such that $\left.\left.s_{j} \in\right] \delta, 1\right]$ for $j \geq j_{0}$, since $\left\{s_{j}\right\}$ converges to 1 . Hence, for $j \geq j_{0}$ we have that $\psi\left(s_{j}\right) \geq \psi(\delta) \geq \psi\left(\frac{\ell_{0}}{\ell_{0}+1}\right)>1-\varepsilon$. Hence, $\psi$ is continuous at $s=1$.

Consider the sequence (harmonic series) $\left\{x_{n}\right\}$ where $x_{n}=\sum_{i=1}^{n} 1 / i$. It is well known that $\left\{x_{n}\right\}$ is a $G$-Cauchy sequence which is not Cauchy in the standard fuzzy metric space $\left(\mathbb{R}, M_{d}, \cdot\right)$ (see [7]). Then it is clear that $\left\{x_{n}\right\}$ is $G$-Cauchy in the stationary fuzzy metric space $\left(X, M_{1}, \cdot\right)$ where $M_{1}(x, y)=$ $\frac{1}{1+d(x, y)}$ and it is almost immediate that $\left\{x_{n}\right\}$ is not Cauchy in $\left(\mathbb{R}, M_{1}, \cdot\right)$. Now we will prove that $\left\{x_{n}\right\}$ is $\psi$-contractive.

For $n \in \mathbb{N}$ we have that

$$
d\left(x_{n}, x_{n+1}\right)=\frac{1}{n+1}, \quad d\left(x_{n+1}, x_{n+2}\right)=\frac{1}{n+2} .
$$

Then,

$$
M_{1}\left(x_{n}, x_{n+1}\right)=\frac{1}{1+\frac{1}{n+1}}=\frac{n+1}{n+2}, \quad M_{1}\left(x_{n+1}, x_{n+2}\right)=\frac{1}{1+\frac{1}{n+2}}=\frac{n+2}{n+3} .
$$

Now, since

$$
\begin{aligned}
\psi\left(M_{1}\left(x_{n}, x_{n+1}\right)\right) & =\psi\left(\frac{n+1}{n+2}\right)=\frac{n+2}{n+4} \cdot \frac{n+1}{n+2}+\frac{2(n+1)+3}{(n+3)(n+4)}= \\
& =\frac{n+1}{n+2} \in\left[\frac{n+1}{n+2}, \frac{n+2}{n+3}[\right.
\end{aligned}
$$

we have that

$$
M_{1}\left(x_{n+1}, x_{n+2}\right)=\frac{n+2}{n+3} \geq \psi\left(M_{1}\left(x_{n}, x_{n+1}\right)\right),
$$

and so $\left\{x_{n}\right\}$ is $\psi$-contractive. 
Remark 3.3.14. The sequence $\left\{x_{n}\right\}$ of Example 3.3 .13 is not strictly fuzzy $\psi$-contractive in $\left(\mathbb{R}, M_{1}, \cdot\right)$. Indeed, $d\left(x_{2}, x_{4}\right)=7 / 12$ and $d\left(x_{3}, x_{5}\right)=9 / 20$. Then $M_{1}\left(x_{3}, x_{5}\right)=20 / 29$ and $M_{1}\left(x_{2}, x_{4}\right)=12 / 19$. Since $12 / 19 \in[1 / 2,2 / 3[$, then

$$
\psi\left(M_{1}\left(x_{2}, x_{4}\right)\right)=\psi(12 / 19)=\frac{2}{4} \cdot \frac{12}{19}+\frac{7}{20}=\frac{373}{380} .
$$

Then, $M_{1}\left(x_{3}, x_{5}\right)=20 / 29<373 / 380=\psi\left(M_{1}\left(x_{2}, x_{4}\right)\right)$, and so, $\left\{x_{n}\right\}$ is not strictly fuzzy $\psi$-contractive. Furthermore, it can be proved that for each $n \in \mathbb{N}$ there exists $m \in \mathbb{N}$ with $m>n$ satisfying $M_{1}\left(x_{n+1}, x_{m+1}\right)<$ $\psi\left(M_{1}\left(x_{n}, x_{m}\right)\right)$. Indeed, fix $n \in \mathbb{N}$. Since $\left\{x_{n}\right\}$ is a sequence of positive terms which diverges to infinity, then we can find $m>n$ satisfying $\sum_{i=n+2}^{m+1} 1 / i \geq 1$. Then,

$$
d\left(x_{n+1}, x_{m+1}\right)=\sum_{i=n+2}^{m+1} \frac{1}{i} \geq 1, \quad d\left(x_{n}, x_{m}\right)=\sum_{i=n+1}^{m} \frac{1}{i}>\sum_{i=n+2}^{m+1} \frac{1}{i} \geq 1 .
$$

Thus,

$$
M_{1}\left(x_{n+1}, x_{m+1}\right)=\frac{1}{1+d\left(x_{n+1}, x_{m+1}\right)} \leq \frac{1}{2}
$$

and

$$
M_{1}\left(x_{n}, x_{m}\right)=\frac{1}{1+d\left(x_{n}, x_{m}\right)}<\frac{1}{2} .
$$

As $M_{1}\left(x_{n}, x_{m}\right) \in\left[0,1 / 2\left[\right.\right.$, we take $\ell=0$ to compute $\psi\left(M_{1}\left(x_{n}, x_{m}\right)\right)$, and so, since $M_{1}\left(x_{n}, x_{m}\right)>0$, we have that

$$
\psi\left(M_{1}\left(x_{n}, x_{m}\right)\right)=\frac{1}{3} M_{1}\left(x_{n}, x_{m}\right)+\frac{1}{2}>\frac{1}{2} \geq M\left(x_{n+1}, x_{m+1}\right) .
$$

Remark 3.3.15. Recently in [46] the following contractive condition, related with the above ones, has been given.

Denote by $\mathcal{Z}$ the family of all functions $\zeta:[0,1] \times] 0,1] \rightarrow \mathbb{R}$ satisfying $\zeta(t, s)>s$ for all $t, s \in] 0,1[$. Let $(X, M, *)$ be a fuzzy metric space and let $f: X \rightarrow X$ be a mapping. Then, $f$ is called a fuzzy $\mathcal{Z}$-contractive mapping with respect to $\zeta$ if $M(f(x), f(y), t) \geq \zeta(M(f(x), f(y), t), M(x, y, t))$ for all 
$x, y \in X, f(x) \neq f(y), t>0$ where $\zeta \in \mathcal{Z}$. According to this definition, we can say that a sequence $\left\{x_{n}\right\}$ in $X$ is $\mathcal{Z}$-contractive with respect to $\zeta \in \mathcal{Z}$ if $M\left(x_{n+1}, x_{n+2}, t\right) \geq \zeta\left(M\left(x_{n+1}, x_{n+2}, t\right), M\left(x_{n}, x_{n+1}, t\right)\right)$ for all $n \in \mathbb{N}$ and $t>0$.

In [46] it is observed that every $\psi$-contractive mapping is fuzzy $\mathcal{Z}$-contractive wih respect to the function $\zeta_{M}$ given by $\zeta_{M}(t, s)=\psi(s)$ for all $\left.\left.s, t \in\right] 0,1\right]$. Then it is immediate that every $\psi$-contractive sequence is $\mathcal{Z}$-contrative sequence with respect to $\zeta_{M}$. Therefore the sequence $\left\{x_{n}\right\}$ constructed in Example 3.3.13 is a fuzzy $\mathcal{Z}$-contractive sequence which is not Cauchy.

\subsection{Correction to Lemma 3.2 of [12]}

Lemma 3.2 of [12] asserts that if $\left\{x_{n}\right\}$ is a $\psi$-contractive sequence satisfying $\bigwedge_{t>0} M\left(x_{1}, x_{2}, t\right)>0$ then $\left\{x_{n}\right\}$ is Cauchy. Clearly, at the light of Example 3.3.13 this assertion is false. Nevertheless one can observe on the proof of this lemma that this assertion is true if we assume that $\left\{x_{n}\right\}$ is strictly fuzzy $\psi$-contractive.

Then, accordingly to the last paragraph, Corollary 3.8 and Lemma 3.12 of [12] must also be corrected as show the next propositions.

Proposition 3.4.1. (Correction of [12, Corollary 3.8]). Let $(X, M, *)$ be a fuzzy metric space such that $\bigwedge_{t>0} M(x, y, t)>0$ for all $x, y \in X$. Then every strictly fuzzy $\psi$-contractive sequence is a Cauchy sequence.

Proposition 3.4.2. (Correction of [12, Lemma 3.12]). Let $(X, M, *)$ be a strong fuzzy metric space. Then every strictly fuzzy $\psi$-contractive sequence is a Cauchy sequence.

Remark 3.4.3. The fixed point theorems of [12] remain valid, since the 
sequence of iterates $\left\{f^{(n)}\left(x_{0}\right)\right\}$ for each $x_{0} \in X$, for a fuzzy $\psi$-contractive mapping $f$ of $X$, is a strictly fuzzy $\psi$-contractive sequence by Proposition 3.3.5.

At the light of Proposition 3.4.2, we propose to investigate the next question.

Question 3.4.4. Is every strictly fuzzy $\psi$-contractive sequence a Cauchy sequence? 


\section{Chapter 4}

\section{Fuzzy partial metric spaces}

\subsection{Introduction}

In this chapter we approach the concept of $(G V$-)fuzzy partial metric as an extension of the concept of partial metric to the fuzzy setting in the sense of Kramosil and Michalek and in the George and Veeramani's one. These extensions have been made in a natural way, but for establishing the triangle inequality we have used the residuum operator $\rightarrow_{*}$ associated to a continuous $t$-norm (Definition 1.1.1). This way of proceeding has been inspired by Demirci [4, Definition 13], without assuming any additional restrictions on the continuous $t$-norm.

We start Section 4.2 defining the concept of $(G V$-)fuzzy partial metric space $(X, P, *)$ (Definitions 4.2.1 and 4.2.2). As in the case of fuzzy metrics, a $G V$-fuzzy partial metric can be regarded as a fuzzy partial metric (Remark 4.2.3). In Propositions 4.2.5 and 4.2.6 we introduce two $G V$-fuzzy partial 
metric spaces, deduced explicitly from a partial metric, while in Example 4.2.9 we introduce a fuzzy partial metric which is not explicitly deduced from a classical partial metric. Example 4.2.7 shows that for a fuzzy partial metric space $(X, P, *)$, it is not true in general that the tern $(X, P, \diamond)$ has to be also a fuzzy partial metric, whenever $\diamond \leq *$. Example 4.2 .10 provides a fuzzy partial metric which is not a $G V$-fuzzy partial metric.

In the beginning of Section 4.3 and motivated by Example 4.3.2, we define the concept of open ball $B_{P}$ centered at $x \in X$, with radius $\left.r \in\right] 0,1[$ and parameter $t \in] 0,+\infty\left[\right.$ as $B_{P}(x, r, t)=\left\{y \in X: P_{x, x, y}^{\prime}(t)>1-r\right\}$, where $P_{x, x, y}^{\prime}(t)=\sup \left\{P(x, x, s) \rightarrow_{*} P(x, y, s): s \in\right] 0, t[\}$. Then, using Lemmas 4.3.4 and 4.3.6 we prove that the family $\mathcal{B}=\left\{B_{P}(x, r, t): x \in X, r \in\right.$ ] $0,1[, t \in] 0,+\infty[\}$ is a base for a topology $\mathcal{T}_{P}$ on $X$, and $\left(X, \mathcal{T}_{P}\right)$ is a $T_{0^{-}}$ space (Theorem 4.3.7). The possibility of refining the concept of open ball in order to obtain a topology on $X$ has ben posed as a question (Question 4.3.8), followed by a justifying remark.

\subsection{Fuzzy partial metric spaces}

The aim of this section is to extend properly the concept of partial metric to the fuzzy setting. First, we will introduce the concept of ( $G V$-)fuzzy partial metric by means of continuous $t$-norms, which will be according to the concept of $(G V$-)fuzzy metric.

Definition 4.2.1. A fuzzy partial metric space is an ordered triple $(X, P, *)$ such that $X$ is a (non-empty) set, $*$ is a continuous $t$-norm and $P$ is a fuzzy set on $X \times X \times[0, \infty[$ satisfying the following conditions, for all $x, y, z \in X$ and $s, t \in] 0, \infty[$ : 
(FPKMO) $P(x, y, 0)=0$;

(FPKM1) $P(x, y, t) \leq P(x, x, t)$;

(FPKM2) $P(x, x, t)=P(y, y, t)=P(x, y, t)$, for each $t \in] 0, \infty[$, if and only if $x=y$;

(FPKM3) $P(x, y, t)=P(y, x, t)$;

(FPKM4) $P(x, x, t+s) \rightarrow_{*} P(x, z, t+s) \geq$ $\left(P(x, x, t) \rightarrow_{*} P(x, y, t)\right) *\left(P(y, y, s) \rightarrow_{*} P(y, z, s)\right) ;$

(FPKM5) The assignment $\left.P_{x, y}:\right] 0, \infty\left[\rightarrow[0,1]\right.$, given by $P_{x, y}(t)=P(x, y, t)$, is a left-continuous function.

According to the notion of $G V$-fuzzy metric space we introduce the following definition.

Definition 4.2.2. A $G V$-fuzzy partial metric space is an ordered triple $(X, P, *)$ such that $X$ is a (non-empty) set, $*$ is a continuous $t$-norm and $P$ is a fuzzy set on $X \times X \times] 0, \infty$ [ satisfying, for all $x, y, z \in X$ and $s, t \in] 0, \infty[$, conditions (FPKM3), (FPKM4) and the following ones:

(FPGV1) $0<P(x, y, t) \leq P(x, x, t)$

(FPGV2) $P(x, x, t)=P(y, y, t)=P(x, y, t)$ if and only if $x=y$;

(FPGV5) The assignment $\left.\left.P_{x, y}:\right] 0, \infty[\rightarrow] 0,1\right]$, given by $P_{x, y}(t)=P(x, y, t)$, is a continuous function.

If $(X, P, *)$ is a $(G V$-)fuzzy partial metric space we will say that $(P, *)$, or simply $P$, is a $(G V$-)fuzzy partial metric on $X$. 
Remark 4.2.3. As in the case of fuzzy metrics, a $G V$-fuzzy partial metric can be regarded as a fuzzy partial one defining $P(x, y, 0)=0$ for each $x, y \in$ $X$. The purpose of this paper is to introduce a topology on a non-empty set $X$ deduced from a $(G V$-)fuzzy partial metric on $X$. Now, as the reader will be able to observe, the value $P(x, y, 0)$ does not play any role in our construction and then the results that we will state for fuzzy partial metric spaces will be also valid for $G V$-fuzzy partial metrics.

Note that the above notions are generalizations of the concepts of fuzzy metric space and $G V$-fuzzy metric space, respectively. Indeed, one can show that a fuzzy metric $(M, *)$ on a non-empty set $X$ is a fuzzy partial metric satisfying the additional condition below, for each $x \in X$ :

$$
M(x, x, t)=1 \text { for each } t \in] 0, \infty[.
$$

After introducing both approaches, to the fuzzy context, of the notion of partial metric, we will justify the axiomatic that has been chosen for them. We only make such observations on the notion of fuzzy partial metric space, since for the George and Veeramani's approach they can be deduced similarly.

Remark 4.2.4. One can observe that the axioms (FPKM1), (FPKM2) and (FPKM3) are "literal" adaptations to the fuzzy context (following the ideas of the notion of fuzzy metric space introduced by Kramosil and Michalek) of axioms (P1), (P2) and (P3), respectively (Definition 1.3.2). Attending to Remark 1.3.3, the (FPKM4) axiom is an adaptation of the inequality (P4). Finally, we have included axiom (FPKM5) for the sake of similarity with fuzzy metric spaces.

After justifying the axioms chosen in the definition of fuzzy partial metric, we continue our study introducing two propositions that provide two examples of $G V$-fuzzy partial metric spaces, which generalize, in some sense, 
the $G V$-fuzzy metric spaces recalled in expressions (1.2.6) and (1.2.5), respectively.

Proposition 4.2.5. Let $(X, p)$ be a partial metric space. We define the fuzzy set on $X \times X \times] 0, \infty[$ as follows

$$
P_{e}(x, y, t)=e^{-\frac{p(x, y)}{t}} .
$$

Then, $\left(X, P_{e}, *_{P}\right)$ is a $G V$-fuzzy partial metric space.

Proof. Let $(X, p)$ be a partial metric space and let $P_{e}$ the function given by

$$
\left.P_{e}(x, y, t)=e^{-p(x, y) / t}, \text { for each } x, y \in X, t \in\right] 0, \infty[\text {. }
$$

Let $x, y, z \in X$ and $t, s \in] 0, \infty\left[\right.$. We will see that $P_{e}$ satisfies all the axioms of Definition 4.2.2.

(FPGV1) Note that $0<e^{-\frac{p(x, y)}{t}} \leq e^{-\frac{p(x, x)}{t}} \leq 1$, since $0 \leq p(x, x) \leq p(x, y)$.

(FPGV2) On the one hand, $P_{e}(x, x, t)=P_{e}(y, y, t)=P_{e}(x, y, t)$ implies $e^{-\frac{p(x, x)}{t}}=$ $e^{-\frac{p(y, y)}{t}}=e^{-\frac{p(x, y)}{t}}$. Then, $p(x, x)=p(y, y)=p(x, y)$ and so $x=y$. On the other hand, if $x=y$, it is obvious that $P_{e}(x, x, t)=P_{e}(y, y, t)=$ $P_{e}(x, y, t)$.

(FPKM3) It is obvious, since $p(x, y)=p(y, x)$.

(FPKM4) Recall that the function $f_{P}$, where $f_{P}(x)=-\log (x)$ for each $x \in[0,1]$, is an additive generator of the usual product $t$-norm (it was observed in [37]). Now, an easy computation shows that the function $f_{P}^{(-1)}$, given by $f_{P}^{(-1)}(y)=e^{-y}$ for each $y \in\left[0, \infty\left[\right.\right.$, is the pseudo-inverse of $f_{P}$.

Let $u, v \in X$ and $r \in] 0, \infty$ [. Attending to formula (1.7) in Section 1.1 we have that

$P_{e}(u, u, r) \rightarrow_{*_{P}} P_{e}(u, v, r)=f_{P}^{(-1)}\left(f_{P}\left(P_{e}(u, v, r)\right)-f_{P}\left(P_{e}(u, u, r)\right)\right)=$ 


$$
\begin{aligned}
= & f_{P}^{(-1)}\left(-\log \left(e^{-\frac{p(u, v)}{r}}\right)+\log \left(e^{-\frac{p(u, u)}{r}}\right)\right)= \\
& f_{P}^{(-1)}\left(\frac{p(u, v)}{r}-\frac{p(u, u)}{r}\right)=e^{-\frac{p(u, v)-p(u, u)}{r}} .
\end{aligned}
$$

Then, using Remark 1.3.3 we have that

$$
\begin{gathered}
P_{e}(x, x, t+s) \rightarrow_{*_{P}} P_{e}(x, z, t+s)=e^{-\frac{p(x, z)-p(x, x)}{t+s}} \geq e^{-\frac{p(x, y)-p(x, x)+p(y, z)-p(y, y)}{t+s}} \geq \\
\quad \geq e^{-\frac{p(x, y)-p(x, x)+p(y, z)-p(y, y)}{\max \{t, s\}}} \geq e^{-\frac{p(x, y)-p(x, x)}{t}} *_{P} e^{-\frac{p(y, z)-p(y, y)}{s}}= \\
\quad=\left(P_{e}(x, x, t) \rightarrow_{*_{P}} P_{e}(x, y, t)\right) *_{P}\left(P_{e}(y, y, s) \rightarrow_{*_{P}} P_{e}(y, z, s)\right) .
\end{gathered}
$$

(FPGV5) Obviously, the function $\left(P_{e}\right)_{x, y}(t)=e^{-\frac{p(x, y)}{t}}$ is a continuous function on $] 0, \infty[$, for each $x, y \in X$.

Hence, $\left(X, P, *_{P}\right)$ is a $G V$-fuzzy partial metric space.

Following the same ideas of the last proof one can prove the following proposition.

Proposition 4.2.6. Let $(X, p)$ be a partial metric space. We define the fuzzy set on $X \times X \times] 0, \infty[$

$$
P_{d}(x, y, t)=\frac{t}{t+p(x, y)} .
$$

Then, $\left(X, P_{d}, *_{H}\right)$ is a $G V$-fuzzy partial metric space.

Proof. Indeed,

(PGV1) As $p(x, x) \leq p(x, y) P(x, x, t)=\frac{t}{t+p(x, x)} \geq \frac{t}{t+p(x, y)}=P(x, y, t)>0$. 
(PGV2) If $x=y$, we have that $P(x, y, t)=\frac{t}{t+p(x, y)}=\frac{t}{t+p(y, y)}=P(y, y, t)$. Analogously, $P(x, y, t)=P(x, x, t)$. On the other hand, if $P(x, y, t)=$ $P(x, x, t)$, we have that $\frac{t}{t+p(x, y)}=\frac{t}{t+p(x, x)}$. Hence, $p(x, y)=p(x, x)$. Analogously, $p(x, y)=p(y, y)$. Therefore, $x=y$.

(PGV3) $P(x, y, t)=\frac{t}{t+p(x, y)}=\frac{t}{t+p(y, x)}=P(y, x, t)$.

(PGV4) We have to show that

$P(x, x, t+s) \rightarrow_{*} P(x, z, t+s) \geq P(x, x, t) \rightarrow_{*} P(x, y, t) *_{H} P(y, y, s) \rightarrow_{*} P(y, z, s)$.

First, we compute $P(x, x, t) \rightarrow_{*} P(x, y, t):$

$$
\begin{aligned}
P(x, x, t) \rightarrow_{*} P(x, y, t)=\sup \{z & {\left.[0,1]: \frac{t}{t+p(x, x)} *_{H} z=\frac{t}{t+p(x, y)}\right\} } \\
\frac{t}{t+p(x, x)} *_{H} z & =\frac{t}{t+p(x, y)} \\
\frac{\frac{t z}{t+p(x, x)}}{z+\frac{t}{t+p(x, x)}-\frac{t z}{t+p(x, x)}} & =\frac{t}{t+p(x, y)} \\
\frac{z}{\frac{t+p(x, x) z}{t+p(x, x)}} & =\frac{t+p(x, x)}{t+p(x, y)} \\
z=\frac{t+p(x, x) z}{t+p(x, y)} & =\frac{t}{t+p(x, y)}+\frac{p(x, x)}{t+p(x, y)} z \\
z\left(1-\frac{p(x, x)}{t+p(x, y)}\right) & =\frac{t}{t+p(x, y)} \\
z\left(\frac{t+p(x, y)-p(x, x)}{t+p(x, y)}\right) & =\frac{t}{t+p(x, y)} \\
z & =\frac{t}{t+p(x, y)-p(x, x)} .
\end{aligned}
$$

Hence, if we denote $q(x, y)=p(x, y)-p(x, x)$, as $q(x, y)$ is the classical quasi-metric deduced from $p$, we have to prove the following inequality:

$$
\begin{aligned}
\frac{t+s}{t+s+q(x, z)} & \geq \frac{t}{t+q(x, y)} * H \frac{s}{s+q(y, z)} \\
& =\frac{t s}{t s+s q(x, y)+t q(y, z)} .
\end{aligned}
$$


By a simple computation, we get

$$
\begin{aligned}
\frac{t+s}{t+s+q(x, z)} & \geq \frac{t s}{t s+s q(x, y)+t q(y, z)} \\
(t+s) t s+(t+s) s q(x, y)+(t+s) t q(y, z) & \geq t s(t+s) t s q(x, z) \\
t s(q(x, y)+q(y, z))+s^{2} q(x, y)+t^{2} q(y, z) & \geq t s q(x, z),
\end{aligned}
$$

which is true because $t s q(x, z) \leq t s(q(x, y)+q(y, z))$. Hence, (PGV4) is satisfied.

(PGV5) The assignment $P(x, y, t)=\frac{t}{t+p(x, y)}$ is clearly continuous for $t>0$.

Hence, $\left(X, *_{H}, P\right)$ is a fuzzy partial metric space.

Moreover, we could show that both examples, presented in Proposition 4.2.5 and Proposition 4.2.6, are $G V$-fuzzy partial metrics for the minimum $t$-norm too. Nevertheless, in the next example we will show that the fuzzy set $P_{d}$ introduced in Proposition 4.2.6 is not a fuzzy partial metric on $X$ for the Lukasievicz $t$-norm, in general.

Example 4.2.7. Let $(X, p)$ be the partial metric space, where $X=[0, \infty[$ and $p(x, y)=\max \{x, y\}$ for each $x, y \in X$, and consider the fuzzy set $P_{d}$ on $X \times X \times] 0, \infty\left[\right.$ given by $P_{d}(x, y, t)=\frac{t}{t+p(x, y)}$. We will see that $\left(X, P_{d}, *_{\mathfrak{L}}\right)$ is not a $G V$-fuzzy partial metric space. Indeed, we will show that $P_{d}$ does not satisfy axiom (FPKM4) for the Lukasievicz $t$-norm.

Let $u, v \in X$ and $r \in] 0, \infty[$. By formula (1.5), we have that

$$
P_{d}(u, u, r) \rightarrow_{*_{\mathfrak{L}}} P_{d}(u, v, r)=1+\frac{r}{r+\max \{u, v\}}-\frac{r}{r+u} .
$$

Now, consider $x=1 ; y=2 ; z=10$ and $t=s=1$. By the last expression,

$P_{d}(x, x, t+s) \rightarrow_{*_{\mathfrak{L}}} P_{d}(x, z, t+s)=1+\frac{1+1}{1+1+10}-\frac{1+1}{1+1+1}=1+\frac{1}{6}-\frac{2}{3}=\frac{1}{2}$, 
and

$$
\begin{aligned}
& \left(P_{d}(x, x, t) \rightarrow_{*_{\mathfrak{L}}} P_{d}(x, y, t)\right) *_{\mathfrak{L}}\left(P_{d}(y, y, s) \rightarrow_{*_{\mathfrak{L}}} P_{d}(y, z, s)\right)= \\
= & \left(1+\frac{1}{1+2}-\frac{1}{1+1}\right)+\left(1+\frac{1}{1+10}-\frac{1}{1+2}\right)-1=\frac{1}{2}+\frac{1}{11} .
\end{aligned}
$$

Therefore, in this case, we have that $P_{d}(x, x, t+s) \rightarrow_{*_{\mathfrak{L}}} P_{d}(x, z, t+s)<$ $\left(P_{d}(x, x, t) \rightarrow_{*_{\mathfrak{L}}} P_{d}(x, y, t)\right) *_{\mathfrak{L}}\left(P_{d}(y, y, s) \rightarrow_{*_{\mathfrak{L}}} P_{d}(y, z, s)\right)$.

Remark 4.2.8. Since $*_{H} \geq *_{\mathcal{L}}$, the preceding example shows a significant difference between fuzzy metrics and fuzzy partial ones. Indeed, given a fuzzy partial metric space $(X, P, *)$ it is not true, in general, that the tern $(X, P, \diamond)$ has to be also a fuzzy partial metric space, whenever $\diamond \leq *$.

The following example shows a fuzzy partial metric which is not explicitly deduced from a classical partial metric.

Example 4.2.9. Let $X=] 0,1]$. We define the fuzzy set $P$ on $X \times X \times[0, \infty[$ by the next expression

$$
P(x, y, t)= \begin{cases}\min \{x, y\} \cdot \frac{t^{2}}{t+1}, & \text { if } x \neq y, t \in] 0,1] \\ \min \{x, y\} \cdot \frac{t}{t+1}, & \text { elsewhere. }\end{cases}
$$

We will see that $(X, P, \wedge)$ is a fuzzy partial metric space.

(FPKM0) By definition, $P(x, y, 0)=\min \{x, y\} \cdot \frac{0}{1}=0$ for every $x, y \in X$.

(FPKM1) Take $x, y \in X$ with $x \neq y$. We will distinguish two cases.

1. Suppose that $t \in] 0,1]$.

First, note that $P(x, x, t)=x \cdot \frac{t}{t+1}<1$ and $P(x, y, t)=\min \{x, y\}$. $\frac{t^{2}}{t+1}>0$.

Then,

$$
1>P(x, x, t)=x \cdot \frac{t}{t+1} \geq \min \{x, y\} \cdot \frac{t^{2}}{t+1}=P(x, y, t)>0 .
$$


2. Now, suppose that $t \in] 1, \infty\left[\right.$. Then, $P(x, x, t)=x \cdot \frac{t}{t+1}<1$ and

$$
\begin{aligned}
& P(x, y, t)=\min \{x, y\} \cdot \frac{t}{t+1}>0, \text { and so } \\
& 1>P(x, x, t)=x \cdot \frac{t}{t+1} \geq \min \{x, y\} \cdot \frac{t}{t+1}=P(x, y, t)>0 .
\end{aligned}
$$

Thus, for each $x, y \in X$ and $t \in] 0, \infty[$ it is satisfied $0<P(x, y, t) \leq$ $P(x, x, t) \leq 1$.

(FPKM2) Obviously, if $x=y$, then $P(x, x, t)=P(y, y, t)=P(x, y, t)$. Conversely, let $x, y \in X$ and $t \in] 0, \infty[$ satisfying $P(x, x, t)=P(y, y, t)=$ $P(x, y, t)$. Then,

$$
P(x, x, t)=x \cdot \frac{t}{t+1}=y \cdot \frac{t}{t+1}=P(y, y, t),
$$

and so $x=y$.

(FPKM3) It is obvious by definition of $P$.

(FPKM4) Let $x, y, z \in X$ and $t, s \in] 0, \infty[$. Suppose that $x \neq z$, since if $x=z$ this axiom is clearly held. We will distinguish two possibilities:

1. Suppose that $t+s \in] 0,1]$. On the one hand, an easy computation shows that

$$
\frac{(t+s)^{2}}{1+t+s} \geq \max \left\{\frac{t^{2}}{1+t}, \frac{s^{2}}{1+s}\right\} .
$$

On the other hand,

$$
P(x, x, t+s) \rightarrow \wedge P(x, z, t+s)=\min \{x, z\} \cdot \frac{(t+s)^{2}}{t+s+1} .
$$

Now, if $x=y$ (or similarly, $y=z)$, then

$$
(P(x, x, t) \rightarrow \wedge P(x, y, t)) \wedge(P(y, y, s) \rightarrow \wedge P(y, z, s))=\min \{x, z\} \cdot \frac{s^{2}}{1+s} .
$$

Thus, (FPKM4) is held. 
Contrary, if $x \neq y$ and $y \neq z$, then

$$
\begin{gathered}
(P(x, x, t) \rightarrow \wedge P(x, y, t)) \wedge(P(y, y, s) \rightarrow \wedge P(y, z, s))= \\
\left(\min \{x, y\} \cdot \frac{t^{2}}{1+t}\right) \wedge\left(\min \{y, z\} \cdot \frac{s^{2}}{s+1}\right) \leq \\
\leq\left(\min \{x, y\} \cdot \max \left\{\frac{t^{2}}{1+t}, \frac{s^{2}}{1+s}\right\}\right) \wedge\left(\min \{y, z\} \cdot \max \left\{\frac{t^{2}}{1+t}, \frac{s^{2}}{1+s}\right\}\right) \leq \\
\leq \min \{x, z\} \cdot \max \left\{\frac{t^{2}}{1+t}, \frac{s^{2}}{1+s}\right\} \leq \min \{x, z\} \cdot \frac{(t+s)^{2}}{1+t+s}= \\
=P(x, x, t+s) \rightarrow \wedge P(x, z, t+s),
\end{gathered}
$$

and so, (FPKM4) is fulfilled too.

2. Suppose that $t+s \in] 1, \infty[$. In such a case, observe that $x \leq z$ implies $P(x, x, t+s)=P(x, z, t+s)$. Then $P(x, x, t+s) \rightarrow \wedge$ $P(x, z, t+s)=1$ and so, the triangle inequality is held. Thus, suppose that $x>z$. Besides, assume that $y \neq x$ and $y \neq z$, since contrary the inequality is fulfilled obviously. On the one hand,

$$
P(x, x, t+s) \rightarrow \wedge P(x, z, t+s)=z \cdot \frac{t+s}{t+s+1} .
$$

On the other hand, we claim that

$$
\begin{aligned}
(P(x, x, t) \rightarrow \wedge P(x, y, t)) & \wedge(P(y, y, s) \rightarrow \wedge P(y, z, s)) \leq \\
& \leq \max \{y, z\} \cdot \min \left\{\frac{t}{t+1} \wedge \frac{s}{s+1}\right\} .
\end{aligned}
$$

To show it, we will distinguish three cases:

(a) Suppose that $y>x$. Then,

$$
P(x, x, t) \rightarrow_{\wedge} P(x, y, t)= \begin{cases}x \cdot \frac{t^{2}}{t+1}, & \text { if } x \neq y, t \in] 0,1] \\ 1, & \text { elsewhere }\end{cases}
$$

and

$$
P(y, y, s) \rightarrow_{\wedge} P(y, z, s)= \begin{cases}z \cdot \frac{s^{2}}{s+1}, & \text { if } x \neq y, s \in] 0,1] \\ z \cdot \frac{s}{s+1}, & \text { elsewhere }\end{cases}
$$


Then, $\left(P(x, x, t) \rightarrow_{\wedge} P(x, y, t)\right) \wedge\left(P(y, y, s) \rightarrow_{\wedge} P(y, z, s)\right) \leq$ $z \cdot \frac{s}{s+1} \leq \min \{y, z\} \cdot \max \left\{\frac{t}{t+1}, \frac{s}{s+1}\right\}$.

(b) Suppose that $x>y>z$. Then,

$$
P(x, x, t) \rightarrow \wedge P(x, y, t)= \begin{cases}y \cdot \frac{t^{2}}{t+1}, & \text { if } x \neq y, t \in] 0,1] ; \\ y \cdot \frac{t}{t+1}, & \text { elsewhere. }\end{cases}
$$

and

$$
P(y, y, s) \rightarrow_{\wedge} P(y, z, s)= \begin{cases}z \cdot \frac{s^{2}}{s+1}, & \text { if } x \neq y, s \in] 0,1] \\ z \cdot \frac{s}{s+1}, & \text { elsewhere. }\end{cases}
$$

Then, $\left(P(x, x, t) \rightarrow_{\wedge} P(x, y, t)\right) \wedge\left(P(y, y, s) \rightarrow_{\wedge} P(y, z, s)\right) \leq$ $z \cdot \frac{s}{s+1} \leq \min \{y, z\} \cdot \max \left\{\frac{t}{t+1}, \frac{s}{s+1}\right\}$.

(c) Suppose that $x>z>y$. Then,

$$
P(x, x, t) \rightarrow_{\wedge} P(x, y, t)= \begin{cases}y \cdot \frac{t^{2}}{t+1}, & \text { if } x \neq y, t \in] 0,1] ; \\ y \cdot \frac{t}{t+1}, & \text { elsewhere. }\end{cases}
$$

and

$$
P(y, y, s) \rightarrow_{\wedge} P(y, z, s)= \begin{cases}y \cdot \frac{s^{2}}{s+1}, & \text { if } x \neq y, s \in] 0,1] \\ 1, & \text { elsewhere. }\end{cases}
$$

Then, $(P(x, x, t) \rightarrow \wedge P(x, y, t)) \wedge\left(P(y, y, s) \rightarrow_{\wedge} P(y, z, s)\right) \leq$ $y \cdot \max \left\{\frac{t}{t+1}, \frac{s}{s+1}\right\} \leq \min \{y, z\} \cdot \max \left\{\frac{t}{t+1}, \frac{s}{s+1}\right\}$.

Therefore, since $z \cdot \frac{t+s}{t+s+1} \geq \min \{y, z\} \cdot \max \left\{\frac{t}{t+1}, \frac{s}{s+1}\right\}$ we have that (FPKM4) is satisfied.

(FPKM5) By definition of $P$, it is easy to verify that $P_{x, y}$ given by $P_{x, y}(t)=$ $P(x, y, t)$, is, obviously, a (non-decreasing) continuous function on $[0,+\infty[$, for each $x, y \in X$.

Hence, $(X, P, \wedge)$ is a fuzzy partial metric space.

The next example provides a fuzzy partial metric which is not a $G V$-fuzzy partial one. 
Example 4.2.10. Let $(X, d)$ be a metric space and let $c \in] 0,1]$. We define the fuzzy set on $X \times X \times[0, \infty[$ as follows

$$
P_{0}(x, y, t)= \begin{cases}0, & \text { if } d(x, y) \geq t \\ c, & \text { if } d(x, y)<t\end{cases}
$$

We will see that $\left(X, P_{0}, \wedge\right)$ is a fuzzy partial metric space.

Proof. Let $x, y, z \in X$ and $t, s \in] 0, \infty\left[\right.$. We will prove that $\left(X, P_{0}, \wedge\right)$ satisfies the axioms of Definition 4.2.1.

(FPKM0) $P_{0}(x, y, 0)=0$ since $d(x, y) \geq 0$ for every $x, y \in X$.

(FPKM1) Observe that $P_{0}(x, x, t)=c$, for each $\left.t \in\right] 0, \infty[$, since $d(x, x)=0<t$. Then, for each $x, y \in X$ and $t \in] 0, \infty\left[\right.$ we have that $P_{0}(x, y, t) \leq$ $P_{0}(x, x, t)$.

(FPKM2) Let $x, y \in X$. On the one hand, by the last observation $P_{0}(x, x, t)=$ $P_{0}(y, y, t)=P_{0}(x, y, t)$ for each $\left.t \in\right] 0, \infty\left[\right.$, implies $P_{0}(x, y, t)=c$ for each $t \in] 0, \infty\left[\right.$, and so, by definition of $P_{0}$ we have that $d(x, y)=0$. Thus $x=y$. On the other hand, if $x=y$, it is obvious that $P_{0}(x, x, t)=$ $P_{0}(y, y, t)=P_{0}(x, y, t)$.

(FPKM3) It is obvious, since $d(x, y)=d(y, x)$.

(FPKM4) First of all, attending to formula (1.3) in Subsection 1.1, by definition of $P_{0}$ we have that

$$
P_{0}(u, u, r) \rightarrow_{\wedge} P_{0}(u, v, r)= \begin{cases}0, & \text { if } d(u, v) \geq r \\ 1, & \text { if } d(u, v)<r\end{cases}
$$

for each $u, v \in X$ and each $r \in] 0, \infty[$. 
Let $M_{0}(x, y, t)=P_{0}(x, x, t) \rightarrow \wedge P_{0}(x, y, t)$. Then, given $x, y, z \in X$ and $t, s \in] 0, \infty[$, we have that

$$
M_{0}(x, z, t+s)= \begin{cases}0, & \text { if } d(x, z) \geq t+s \\ 1, & \text { if } d(x, z)<t+s,\end{cases}
$$

Therefore, if $d(x, z)<t+s$, the inequality

$$
M_{0}(x, z, t+s) \geq M_{0}(x, y, t) \wedge M(y, z, s)
$$

is fulfilled for every $x, y, z \in X$ and $t, s>0$. Alternatively, if $d(x, y) \geq$ $t+s$, since $d(x, z) \leq d(x, y)+d(y, z)$ for every $x, y, z \in X$, we have that $d(x, y) \geq t$ or $d(y, z) \geq s$. In that case, $M_{0}(x, y, t)=0$ or $M_{0}(y, z, s)=$ 0. Hence, (FPKM4) is also satisfied.

(FPKM5) By definition, the assignment $\left(P_{0}\right)_{x, y}(t)$ is a left-continuous function on ] $0, \infty[$, for each $x, y \in X$.

However, $\left(P_{0}\right)_{x, y}$ is not continuous whenever $x \neq y$, and consequently $P_{0}$ is not a $G V$-fuzzy partial metric.

We finish this section providing another significant difference between $(G V$-)fuzzy metrics and ( $G V$-)fuzzy partial metrics. Such a difference is given by the fact that, for each $x, y \in X$, the function $P_{x, y}$ defined in the axiom (FPKM5) is not increasing, in general.

Example 4.2.11. Consider the set $X=\mathbb{R}$ and the usual product $t$-norm $*_{P}$. We define a fuzzy set $P$ on $X \times X \times[0, \infty[$ given by

$$
P(x, y, t)= \begin{cases}0, & \text { if } t=0 \\ e^{-t}, & \text { if } x=y \\ \frac{1}{2} e^{-t}, & \text { if } x \neq y\end{cases}
$$


It is not hard to check that $\left(X, P, *_{P}\right)$ is a fuzzy partial metric space. Indeed, (FPKM0)-(FPKM3) are obviously fulfilled by $P$ attending to its definition. To show (FPKM4), consider $x, y, z \in X$, with $x \neq z$ (since, contrarily, $P(x, x, t+s) \rightarrow_{*_{P}} P(x, z, t+s)=1$ and so, the inequality is satisfied) and $t, s \in] 0, \infty[$. On account of formula (1.4) in Subsection 1.1,

$$
P(x, x, t+s) \rightarrow_{*_{P}} P(x, z, t+s)=\frac{1}{2} .
$$

Now, by our assumption, at least $x \neq y$ or $z \neq y$, and so

$$
\left(P(x, x, t) \rightarrow_{*_{P}} P(x, y, t)\right) *_{P}\left(P(y, y, s) \rightarrow_{*_{P}} P(y, z, s)\right) \leq \frac{1}{2} .
$$

Therefore, (FPKM4) is also held.

Obviously, $P_{x, y}$ is a continuous function for each $x, y \in X$, which is not increasing.

\subsection{Topology induced by a fuzzy partial metric}

The aim of this section is to define a topology deduced from a fuzzy partial metric. Such a topology is defined by means of open balls, in a similar way to the classical case. Based on the notions of open ball, both in partial metrics and fuzzy ones, it seems natural to define the concept of open ball in a fuzzy partial metric space as follows:

Definition 4.3.1. Let $(X, P, *)$ be a fuzzy metric space, the open ball $\tilde{B}_{P}$ centred at $x \in X$, with radius $r \in] 0,1[$ and parameter $t \in] 0, \infty[$ is defined by the next expression:

$$
\tilde{B}_{P}(x, r, t)=\left\{y \in X: P(x, x, t) \rightarrow_{*} P(x, y, t)>1-r\right\} .
$$


Observe that, for each $x, y \in X$, the assignment $\left.P_{x, x, y}:\right] 0, \infty[\rightarrow[0,1]$ is a well-defined function, where $P_{x, x, y}$ is given by

$$
\left.P_{x, x, y}(t)=P(x, x, t) \rightarrow_{*} P(x, y, t), \text { for each } t \in\right] 0, \infty[.
$$

Indeed, $P_{x, x, y}(t)=\sup \{c \in[0,1]: P(x, x, t) * c=P(x, y, t)\}$, which is unique. Furthermore, as a consequence of the axiom (FPKM4) in Definition 4.2.1, we can deduce that, for each $x, y \in X$, the function $P_{x, x, y}$ is nondecreasing. However, such a function is not left-continuous in general, as shows the following example.

Example 4.3.2. Let $(X, P, \wedge)$ be the fuzzy partial metric space of Example 4.2.9. Consider $x=\frac{1}{4}$ and $y=\frac{1}{2}$. Then, $P(x, x, t)=\frac{t}{4(t+1)}$ for each $\left.t \in\right] 0, \infty[$ and

$$
P(x, y, t)= \begin{cases}\frac{t^{2}}{4(t+1)}, & \text { if } t \in] 0,1] ; \\ \frac{t}{4(t+1)}, & \text { elsewhere. }\end{cases}
$$

Therefore, attending to equation (1.3) the function $P_{x, x, y}$ is given by

$$
P_{x, x, y}(t)= \begin{cases}\frac{t^{2}}{4(t+1)}, & \text { if } t \in] 0,1[; \\ 1, & \text { if } t \in[1, \infty[,\end{cases}
$$

which is not left-continuous at $t=1$.

In our next construction of a topology $\mathcal{T}_{P}$ deduced from $P$ we shall need left-continuity for $P_{x, x, y}$. To overcome this lack of left-continuity of the function $P_{x, x, y}$, in general, we propose to define the open balls in a fuzzy partial metric space as follows.

Definition 4.3.3. Let $(X, P, *)$ be a fuzzy partial metric space. We define the open ball $B_{P}$ centred at $x \in X$, with radius $\left.r \in\right] 0,1$ [ and parameter $t \in] 0, \infty[$ by the next expression:

$$
B_{P}(x, r, t)=\left\{y \in X: P_{x, x, y}^{\prime}(t)>1-r\right\},
$$

where $P_{x, x, y}^{\prime}(t)=\sup \left\{P(x, x, s) \rightarrow_{*} P(x, y, s): s \in\right] 0, t[\}$. 
Attending to the above observations on the assignment $P_{x, x, y}(t)$, it is not hard to check that $P_{x, x, y}^{\prime}$ is a non-decreasing function on $] 0, \infty[$, for each $x, y \in X$. Furthermore, $P_{x, x, y}^{\prime}$ is left-continuous, for each $x, y \in X$, as shows the next lemma.

Lemma 4.3.4. Let $(X, P, *)$ be a fuzzy partial metric space. For each $x, y \in$ $X$, the function $\left.P_{x, x, y}^{\prime}:\right] 0, \infty[\rightarrow[0,1]$ given by

$$
\left.P_{x, x, y}^{\prime}(t)=\sup \left\{P(x, x, s) \rightarrow_{*} P(x, y, s): s \in\right] 0, t[\}, \text { for each } t \in\right] 0, \infty[,
$$

is left-continuous.

Proof. Take $x, y \in X$. We define, for each $t \in] 0, \infty[$, the function

$$
P_{x, x, y}^{\prime}(t)=\sup \left\{P(x, x, s) \rightarrow_{*} P(x, y, s): s \in\right] 0, t[\} .
$$

Let $\left.t_{0} \in\right] 0, \infty\left[\right.$. Since $\left(P\left(x, x, s_{2}\right) \rightarrow_{*} P\left(x, y, s_{2}\right)\right) \geq\left(P\left(x, x, s_{1}\right) \rightarrow_{*} P\left(x, y, s_{1}\right)\right)$, whenever $\left.s_{1}, s_{2} \in\right] 0, \infty\left[\right.$ with $s_{2}>s_{1}$, and attending to the definition of $P_{x, x, y}^{\prime}$, we deduce that for each $\epsilon>0$ there exists $\left.s_{0} \in\right] 0, t_{0}[$ such that

$$
P\left(x, x, s_{0}\right) \rightarrow_{*} P\left(x, y, s_{0}\right)>P_{x, x, y}^{\prime}\left(t_{0}\right)-\epsilon .
$$

Then, by the last inequality, we have that

$$
P(x, x, s) \rightarrow_{*} P(x, y, s)>P_{x, x, y}^{\prime}\left(t_{0}\right)-\epsilon, \text { for each } s \in\left[s_{0}, t_{0}\right] .
$$

Therefore, given $\epsilon>0$ there exists $\delta>0$, with $\delta=t_{0}-s_{0}$, such that

$$
P_{x, x, y}^{\prime}(t)=\sup \left\{P(x, x, s) \rightarrow_{*} P(x, y, s): s \in\right] 0, t[\}>P_{x, x, y}^{\prime}\left(t_{0}\right)-\varepsilon,
$$

for each $\left.t \in] t_{0}-\delta, t_{0}\right]$. Thus, $P_{x, x, y}^{\prime}$ is left continuous at $t_{0}$, and since $t_{0}$ is arbitrary then $P_{x, x, y}^{\prime}$ is left-continuous on $] 0, \infty[$.

Remark 4.3.5. Observe that if, for each $x, y \in X$, the function $P_{x, x, y}$ is left-continuous, then $\tilde{B}_{P}(x, r, t)=B_{P}(x, r, t)$, for each $\left.x \in X, r \in\right] 0,1[$ and $t \in] 0, \infty[$. 
Lemma 4.3.6. Let $(X, P, *)$ be a fuzzy partial metric space. Then, for each $x, y, z \in X$ and each $t, s \in] 0, \infty[$ it is satisfied the following:

$$
P_{x, x, z}^{\prime}(t+s) \geq P_{x, x, y}^{\prime}(t) * P_{y, y, z}^{\prime}(s) .
$$

Proof. Let $x, y, z \in X$ and $t, s \in] 0, \infty\left[\right.$. Consider $\left.t^{\prime} \in\right] 0, t\left[\right.$ and $\left.s^{\prime} \in\right] 0, s[$. Then,

$$
\begin{aligned}
P_{x, x, z}^{\prime}(t+s) & \geq P\left(x, x, t^{\prime}+s^{\prime}\right) \rightarrow_{*} P\left(x, z, t^{\prime}+s^{\prime}\right) \\
& \geq\left(P\left(x, x, t^{\prime}\right) \rightarrow_{*} P\left(x, y, t^{\prime}\right)\right) *\left(P\left(y, y, s^{\prime}\right) \rightarrow_{*} P\left(y, z, s^{\prime}\right)\right) .
\end{aligned}
$$

Since $\left.t^{\prime} \in\right] 0, t\left[\right.$ and $\left.s^{\prime} \in\right] 0, s[$ are arbitrary then

$$
\begin{aligned}
P_{x, x, z}^{\prime}(t+s) & \geq \sup _{0<t^{\prime}<t}\left\{P\left(x, x, t^{\prime}\right) \rightarrow_{*} P\left(x, y, t^{\prime}\right)\right\} * \sup _{0<s^{\prime}<s}\left\{P\left(y, y, s^{\prime}\right) \rightarrow_{*} P\left(y, z, s^{\prime}\right)\right\} \\
& =P_{x, x, y}^{\prime}(t) * P_{y, y, z}^{\prime}(s) .
\end{aligned}
$$

Now, we are able to show the main goal of this section. We have included the proof for the sake of completeness and because of its particularities.

Theorem 4.3.7. Let $(X, P, *)$ be a fuzzy partial metric space. Then, the family of open balls

$$
\mathcal{B}=\left\{B_{P}(x, r, t): x \in X, r \in\right] 0,1[, t \in] 0, \infty[\}
$$

forms a base of a topology $\mathcal{T}_{P}$, which is $T_{0}$.

Proof. Let $\left.x_{1}, x_{2} \in X, r_{1}, r_{2} \in\right] 0,1\left[, t_{1}, t_{2} \in\right] 0, \infty[$ and consider the open balls $B_{P}\left(x_{1}, r_{1}, t_{1}\right)$ and $B_{P}\left(x_{2}, r_{2}, t_{2}\right)$. 
We will show that for each $y \in B_{P}\left(x_{1}, r_{1}, t_{1}\right) \cap B_{P}\left(x_{2}, r_{2}, t_{2}\right)$ we can find $r \in] 0,1[$ and $t \in] 0, \infty\left[\right.$ satisfying $B_{P}(y, r, t) \subset B_{P}\left(x_{1}, r_{1}, t_{1}\right) \cap B_{P}\left(x_{2}, r_{2}, t_{2}\right)$.

Let $y \in B_{P}\left(x_{1}, r_{1}, t_{1}\right) \cap B_{P}\left(x_{2}, r_{2}, t_{2}\right)$. Then, by definition of open ball, we have that

$$
P_{x_{1}, x_{1}, y}^{\prime}\left(t_{1}\right)>1-r_{1} \quad \text { and } \quad P_{x_{2}, x_{2}, y}^{\prime}\left(t_{2}\right)>1-r_{2} .
$$

On the one hand, we can find $\left.r_{1}^{\prime} \in\right] 0, r_{1}\left[\right.$ and $\left.r_{2}^{\prime} \in\right] 0, r_{2}[$ such that

$$
P_{x_{1}, x_{1}, y}^{\prime}\left(t_{1}\right)>\left(1-r_{1}^{\prime}\right) \quad \text { and } \quad P_{x_{2}, x_{2}, y}^{\prime}\left(t_{2}\right)>\left(1-r_{2}^{\prime}\right)
$$

And, on the other hand, since the functions $P_{x_{1}, x_{1}, y}^{\prime}$ and $P_{x_{2}, x_{2}, y}^{\prime}$ are nondecreasing left-continuous functions on $] 0, \infty\left[\right.$, then we can find $\left.t_{1}^{\prime} \in\right] 0, t_{1}$ [ and $\left.t_{2}^{\prime} \in\right] 0, t_{2}[$ satisfying

$$
P_{x_{1}, x_{1}, y}^{\prime}\left(t_{1}^{\prime}\right)>\left(1-r_{1}^{\prime}\right) \quad \text { and } \quad P_{x_{2}, x_{2}, y}^{\prime}\left(t_{2}^{\prime}\right)>\left(1-r_{2}^{\prime}\right)
$$

Now, since $*$ is continuous, there exist $\left.r_{1}^{\prime \prime} \in\right] 0,1\left[\right.$ and $\left.r_{2}^{\prime \prime} \in\right] 0,1[$ such that $\left(1-r_{1}^{\prime}\right) *\left(1-r_{1}^{\prime \prime}\right)>\left(1-r_{1}\right)$ and $\left(1-r_{2}^{\prime}\right) *\left(1-r_{2}^{\prime \prime}\right)>1-r_{2}$, respectively.

Take $r=\min \left\{r_{1}^{\prime \prime}, r_{2}^{\prime \prime}\right\}$ and $t=\min \left\{t_{1}-t_{1}^{\prime}, t_{2}-t_{2}^{\prime}\right\}$. We will see that $B_{P}(y, r, t) \subset B_{P}\left(x_{1}, r_{1}, t_{1}\right) \cap B_{P}\left(x_{2}, r_{2}, t_{2}\right)$. Indeed, take $z \in B_{P}(y, r, t)$, then applying Lemma 4.3.6 in the first inequality and the monotony of the function $P_{x_{1}, x_{1}, y}^{\prime}$ in the second one, we have that

$$
\begin{aligned}
P_{x_{1}, x_{1}, z}^{\prime}\left(t_{1}\right) & \geq\left(P_{x_{1}, x_{1}, y}^{\prime}\left(t_{1}^{\prime}\right)\right) *\left(P_{y, y, z}^{\prime}\left(t_{1}-t_{1}^{\prime}\right)\right) \geq\left(P_{x_{1}, x_{1}, y}^{\prime}\left(t_{1}^{\prime}\right)\right) *\left(P_{y, y, z}^{\prime}(t)\right) \geq \\
& \geq\left(1-r_{1}^{\prime}\right) *(1-r) \geq\left(1-r_{1}^{\prime}\right) *\left(1-r_{1}^{\prime \prime}\right)>\left(1-r_{1}\right),
\end{aligned}
$$

and so $z \in B_{P}\left(x_{1}, r_{1}, t_{1}\right)$.

Analogously, one can prove that $z \in B_{P}\left(x_{2}, r_{2}, t_{2}\right)$. Hence, $\mathcal{B}$ is a base of a topology $\mathcal{T}_{P}$. 
Finally, we will prove that $\left(X, \mathcal{T}_{P}\right)$ is a $T_{0}$-space. Indeed, if $x \neq y$, there exists $\left.t_{0} \in\right] 0, \infty\left[\right.$ such that $P_{x, x, y}^{\prime}\left(t_{0}\right)<1$ or $P_{y, y, x}^{\prime}\left(t_{0}\right)<1$. Contrary, $P_{x, x, y}^{\prime}(t)=P_{x, x, y}^{\prime}(t)=1$, for each $\left.t \in\right] 0, \infty[$, that implies $P(x, x, t)=$ $P(x, y, t)=P(y, y, t)$, for each $t \in] 0, \infty[$, which is equivalent to $x=y$. Suppose that $P_{x, x, y}^{\prime}\left(t_{0}\right)=1-r_{0}<1$. Let $r_{1}<r_{0}<1$. Then $y \notin B_{P}\left(x, r_{1}, t_{0}\right)$, and so $\left(X, \mathcal{T}_{P}\right)$ is a $T_{0}$-space.

Concerning the above theorem, given a fuzzy partial metric $P$ on a nonempty set $X$, we will say that $\mathcal{T}_{P}$ is the topology deduced from $P$ or induced by $P$.

Coming back to the fuzzy partial metric case, as it has been shown in Example 4.3.2, axioms (FPKM1)-(PKM5) do not ensure the left-continuity of the function $P_{x, x, y}$. Nevertheless, in such an example, one can verify that we are able to define a topology on $X$ deduced from $P$, which has as a base the family of open balls $\tilde{B}_{P}$. It is due to the fact that the fuzzy partial metric $P$, defined in Example 4.2.9, satisfies in addition the next condition, for each $x, y, z \in X$ and each $t \in] 0, \infty[$, $P(x, x, t) \rightarrow_{*} P(x, z, t) \geq\left(P(x, x, t) \rightarrow_{*} P(x, y, t)\right) *\left(P(y, y, t) \rightarrow_{*} P(y, z, t)\right)$. So, the above comments motivate the next question.

Question 4.3.8. Let $(X, P, *)$ be a fuzzy metric space. Can we define a topology on $X$ which has as a base the family

$$
\left\{\tilde{B}_{P}(x, r, t): x \in X, r \in\right] 0,1[, t \in] 0, \infty[\} ?
$$

Remark 4.3.9. Example 4.3.2 shows a fuzzy partial metric such that $P_{x, x, y}$ is not a left-continuous function but the family

$$
\left\{\tilde{B}_{P}(x, r, t): x \in X, r \in\right] 0,1[, t \in] 0, \infty[\}
$$

defines a topology on $X$. 


\section{Chapter 5}

\section{A duality relationship between fuzzy partial metrics and fuzzy quasi-metrics}

\subsection{Introduction}

In addition to introduce the notion of partial metric in [6], Matthews showed a duality relationship between partial metrics and quasi-metrics. Such a relationship is given by the fact that, from each partial metric $p$ on a nonempty set $X$ we can construct a quasi-metric $q_{p}$ on $X$ defining $q_{p}(x, y)=$ $p(x, y)-p(x, x)$, for each $x, y \in X$. In order to obtain a similar construction in the converse case, Matthews introduced the notion of weighted quasimetric space. Following [6], a weighted quasi-metric space is a tern $(X, q, w)$, where $q$ is a quasi-metric on $X$ and $w$ is a function defined on $X$ satisfying, for each $x, y \in X$, the following conditions: 
(w1) $w(x) \geq 0$

(w2) $q(x, y)+w(x)=q(y, x)+w(y)$.

Then, Matthews established a way to construct a partial metric from a given weighted quasi-metric space $(X, q, w)$, defining a partial metric $p_{q}$ on $X$ given by $p_{q}(x, y)=q(x, y)+w(x)$, for each $x, y \in X$.

Besides, Matthews showed that both constructions preserve the topology. Indeed, given a partial metric space $(X, p)$ then, $\mathcal{T}(p)=\mathcal{T}\left(q_{p}\right)$. Conversely, given a weighted quasi-metric space $(X, q, w)$ then, $\mathcal{T}(q)=\mathcal{T}\left(p_{q}\right)$.

Based on the duality relationship exposed above, the aim of this chapter is to retrieve the above relationship in the fuzzy setting. On account of the definition of fuzzy partial metric space introduced in Chapter 4, given a fuzzy partial metric $(P, *)$ on $X, P(x, y, t)$ can take the value 0 for some $x, y \in X$ and $t>0$. This fact can be translated to the classical case assuming that, given a partial metric $p$ on $X, p(x, y)$ can take the value $\infty$ for some $x, y \in X$. In its study, Matthews did not consider such a case. So, we have avoided to consider the Kramosil and Michalek's sense to provide a first approach to retrieve the classical relationship between partial metrics and quasi-metrics.

Hence, in Section 5.2 we focus on the notion of $G V$-fuzzy partial metric introduced in Chapter 4 and a concept of fuzzy quasi-metric given by Gregori and Romaguera in [24] (Definition 5.2.1). We introduce two examples of $G V$-fuzzy metrics that are a generalization of the standard and exponential fuzzy metrics (Example 5.2.2), and then we give a way to define a $G V$-fuzzy quasi-metric $\left(X, Q_{P}, *\right)$ from a $G V$-fuzzy partial metric $(X, P, *)$, where * is a continuous, Archimedean $t$-norm (Theorem 5.2.3). Also, we prove, as a consequence of Proposition 5.2.5, that the topology induced by this $G V$ fuzzy quasi-metric is the same as the topology induced by the $G V$-fuzzy partial metric space. 
In Section 5.3, we generalize the concept of weighted quasi-metric to the fuzzy context (Definition 5.3.1), in order to give a way to define a $G V$-fuzzy partial metric from a $G V$-fuzzy weighted quasi-metric space (Theorem 5.3.4). Through the chapter, the continuous $t$-norm that we use to define the $G V$ fuzzy partial metric and quasi-metric spaces is demanded to be Archimedean. This fact is justified in Example 5.3.6. Moreover, it is proved that the $G V$-fuzzy quasi-metric deduced from a $G V$-fuzzy partial metric is weighable (Theorem 5.3.7) and thus, both Sections 5.2 and 5.3 define a duality between $G V$-fuzzy partial metric spaces and $G V$-fuzzy quasi-metric spaces.

\subsection{From $G V$-fuzzy partial metrics to $G V$-fuzzy (quasi-)metrics}

In this section, we provide a way of constructing a $G V$-fuzzy quasi-metric from a $G V$-fuzzy partial metric. To get such an aim, we are based on the classical techniques given by Matthews in [6].

We begin recalling the concept of $G V$-fuzzy quasi-metric introduced by Gregori and Romaguera [24].

Definition 5.2.1. A $G V$-fuzzy quasi-metric space is a tern $(X, Q, *)$ such that $X$ is a non-empty set, $*$ is a continuous $t$-norm and $Q$ is a fuzzy set on $X \times X \times] 0,+\infty)$ satisfying the following conditions, for all $x, y, z \in X$ and $t, s \in] 0, \infty[:$

(QGV1) $Q(x, y, t)>0$

(QGV2) $Q(x, y, t)=Q(y, x, t)=1$ if and only if $x=y$;

(QGV3) $Q(x, z, t+s) \geq Q(x, y, t) * Q(y, z, s)$; 
(QGV4) The assignment $\left.\left.Q_{x, y}:\right] 0,+\infty[\rightarrow] 0,1\right]$, given by $Q_{x, y}(t)=Q(x, y, t)$ for each $t \in] 0,+\infty[$, is a continuous function.

In such a case, $(Q, *)$, or simply $Q$, is called a $G V$-fuzzy quasi-metric on $X$.

In addition, Gregori and Romaguera proved in [24] that every fuzzy quasimetric $Q$ on $X$ generates a $T_{0}$ topology $\mathcal{T}_{Q}$ on $X$ which has as a base the family of open sets of the form $\left\{B_{Q}(x, \epsilon, t): x \in X, 0<\epsilon<1, t>0\right\}$, where $B_{Q}(x, \epsilon, t)=\{y \in X: Q(x, y, t)>1-\epsilon\}$ for all $\left.x \in X, \epsilon \in\right] 0,1[$ and $t>0$.

Before providing the aformetnioned construction, we introduce two examples of $G V$-fuzzy quasi-metric spaces. They generalize, in some sense, the standard and exponential fuzzy metric spaces deduced from a classic metric (see [7]). Both examples will be useful later.

Example 5.2.2. (i) Let $(X, q)$ be a quasi-metric space. We define the fuzzy set $Q_{e}$ on $\left.X \times X \times\right] 0, \infty[$ as follows

$$
Q_{e}(x, y, t)=e^{-\frac{q(x, y)}{t}} .
$$

Then, $\left(X, Q_{e}, \wedge\right)$ is a $G V$-fuzzy partial metric space which we call exponential $G V$-fuzzy quasi-metric space deduced from $q$.

We will see that $Q_{e}$ satisfies, for each $x, y, z \in X$ and $t>0$, axioms $(Q G V 1)-(Q G V 4)$.

$(\mathrm{QGV} 1) Q_{e}(x, y, t)=e^{-\frac{q(x, y)}{t}}>0$ for every $x, y \in X, t>0$.

(QGV2) If $x=y$, then

$$
\begin{aligned}
& Q_{e}(x, y, t)=e^{-\frac{q(x, y)}{t}}=e^{0}=1, \\
& Q_{e}(y, x, t)=e^{-\frac{q(y, x)}{t}}=e^{0}=1 .
\end{aligned}
$$


Therefore $Q_{e}(x, y, t)=Q_{e}(y, x, t)=1$. Conversely, if $Q_{e}(x, y, t)=$ $Q_{e}(y, x, t)=1$, then

$$
\begin{aligned}
e^{-\frac{q(x, y)}{t}} & =e^{-\frac{q(y, x)}{t}}=1, \\
\frac{q(x, y)}{t} & =\frac{q(y, x)}{t}=0, \\
q(x, y) & =q(y, x)=0,
\end{aligned}
$$

hence $x=y$.

(QGV3) We have to prove that $Q_{e}(x, z, t+s) \geq Q_{e}(x, y, t) \wedge Q_{e}(y, z, s)$.

Suppose that $Q_{e}(x, y, t) \geq Q_{e}(y, z, s)$. Then, $e^{-\frac{q(x, y)}{t}} \geq e^{-\frac{q(y, z)}{s}}$, or equivalently, $s q(x, y) \leq t q(y, z)$. So, in this case, the next inequality

$$
Q_{e}(x, z, t+s) \geq Q_{e}(x, y, t) \wedge Q_{e}(y, z, s)=Q_{e}(y, z, s),
$$

is satisfied, if and only if, it is fulfilled the following one:

$$
e^{-\frac{q(x, z)}{t+s}} \geq e^{-\frac{q(y, z)}{s}}
$$

Observe that the previous inequality is held, if and only if,

$$
\frac{q(x, z)}{t+s} \leq \frac{q(y, z)}{s}(\Leftrightarrow s q(x, z) \leq t q(y, z)+s q(y, z)) .
$$

Since $q$ is a quasi-metric and $s q(x, y) \leq t q(y, z)$ we have that $s q(x, z) \leq s q(x, y)+s q(y, z) \leq t q(y, z)+s q(y, z)$.

Thus, $Q_{e}(x, z, t+s) \geq Q_{e}(x, y, t) \wedge Q_{e}(y, z, s)$ in this case.

The case $Q_{e}(x, y, t) \leq Q_{e}(y, z, s)$ is proved analogously.

(QGV4) The assignment $\left(Q_{e}\right)_{x y}(t)=e^{-\frac{q(x, y)}{t}}$ is continuous for every $t>0$.

(ii) Let $(X, q)$ be a quasi-metric space. We define the fuzzy set $Q_{d}$ on $X \times X \times] 0, \infty[$ as

$$
Q_{d}(x, y, t)=\frac{t}{t+q(x, y)} .
$$


Then, $\left(X, Q_{d}, *_{P}\right)$ is a $G V$-fuzzy quasi-metric space (see [24]). Following similar arguments to the ones used in $(i)$, one can show that $\left(X, Q_{d}, \wedge\right)$ is also a $G V$-fuzzy quasi-metric space.

Now, we are able to show the next theorem.

Theorem 5.2.3. Let $(X, P, *)$ be a $G V$-fuzzy partial metric space, where * is a continuous Archimedean t-norm. Then, $\left(X, Q_{P}, *\right)$ is a $G V$-fuzzy quasimetric space, where $Q_{P}$ is the fuzzy set on $\left.X \times X \times\right] 0, \infty[$ given by:

$$
Q_{P}(x, y, t)=P(x, x, t) \rightarrow_{*} P(x, y, t)
$$

for each $x, y \in X, t>0$.

Proof. We will show that every axiom of Definition 5.2.1 is fulfilled:

(QGV1) As $P(x, y, t)>0$, then $P(x, x, t) \rightarrow_{*} P(x, y, t)=\sup \{z \in[0,1]:$ $P(x, x, t) * z=P(x, y, t)\}>0$. Hence $Q_{P}(x, y, 0)>0$.

(QGV2) $x=y$ implies that $P(x, x, t)=P(x, y, t)$ and $P(y, y, t)=P(y, x, t)$ for each $t>0$. Hence, $Q_{P}(x, y, t)=P(x, x, t) \rightarrow_{*} P(x, y, t)=1$ and $Q_{P}(y, x, t)=P(y, y, t) \rightarrow_{*} P(y, x, t)=1$. Therefore, $Q_{P}(x, y, t)=1$ and $Q_{P}(y, x, t)=1$. On the other hand, if $Q_{P}(x, y, t)=Q_{P}(y, x, t)=1$ for some $t>0$, we have that $P(x, x, t) \rightarrow_{*} P(x, y, t)=P(y, y, t) \rightarrow_{*}$ $P(y, x, t)=1$. Hence, as $P(x, x, t) \geq P(x, y, t)$ and $P(y, y, t) \geq$ $P(y, x, t)$, we have that $P(x, x, t)=P(x, y, t)=P(y, x, t)=P(y, y, t)$ for some $t>0$, and so $x=y$.

(QGV3) It is straightforward due to axiom (PGV4).

$\left(\right.$ QGV4) $\left(Q_{P}\right)_{x y}(t)=Q(x, y, t)=P(x, x, t) \rightarrow_{*} P(x, y, t)$ is a continuous function as we have shown in Remark 1.1.6. 
The condition imposed on the $t$-norm, which is that it must be Archimedean, cannot be removed to prove the previous theorem, as shows the next example.

Example 5.2.4. Let $(X, P, \wedge)$ be the fuzzy partial metric space of Example 4.2.9, where Let $X=] 0,1]$ and $P$ is the fuzzy set defined on $X \times X \times] 0, \infty[$ by the following expression

$$
P(x, y, t)= \begin{cases}\min \{x, y\} \cdot \frac{t^{2}}{t+1}, & \text { if } x \neq y, t \in] 0,1] \\ \min \{x, y\} \cdot \frac{t}{t+1}, & \text { elsewhere. }\end{cases}
$$

It is not hard to check that $(X, P, \wedge)$ is a $G V$-fuzzy partial metric space. Nevertheless, if we define the fuzzy set $Q_{P}$ on $\left.X \times X \times\right] 0, \infty\left[\right.$ by $Q_{P}(x, y, t)=$ $P(x, x, t) \rightarrow_{\wedge} P(x, y, t)$, for each $x, y \in X$ and $t>0$, then $Q_{P}$ does not satisfy axiom ( $Q G V 4)$. Indeed, on account of Example 4.3.2 we have that

$$
Q_{P}\left(\frac{1}{4}, \frac{1}{2}, t\right)=P\left(\frac{1}{4}, \frac{1}{4}, t\right) \rightarrow \wedge P\left(\frac{1}{4}, \frac{1}{2}, t\right)= \begin{cases}\frac{t^{2}}{4(t+1)}, & \text { if } t \in] 0,1[ \\ 1, & \text { if } t \in[1, \infty[\end{cases}
$$

Obviously, $\left(Q_{P}\right)_{\frac{1}{4}, \frac{1}{2}}$ is not a continuous function.

We motivate the construction presented in Theorem 5.2.3 applying it to the examples of $G V$-fuzzy partial metric spaces introduced in Proposition 4.2.5 and 4.2.6.

Let $(X, p)$ be a partial metric space. Following the Matthews' construction we have that $q_{p}$ is a quasi-metric on $X$, where $q_{p}(x, y)=p(x, y)-p(x, x)$ for each $x, y \in X$.

On the one hand, by Proposition 4.2.5, $\left(X, P_{e}, *_{P}\right)$ is a $G V$-fuzzy partial metric space, where $P_{e}(x, y, t)=e^{-\frac{p(x, y)}{t}}$, for each $x, y \in X, t>0$. Since $*_{P}$ 
is a continuous Archimedean $t$-norm then, by Theorem 5.2.3, we have that $\left(X, Q_{P_{e}}, *_{P}\right)$ is a $G V$-fuzzy quasi-metric space, where $Q_{P_{e}}$ is given by

$$
Q_{P_{e}}(x, y, t)=P_{e}(x, x, t) \rightarrow_{*} P_{e}(x, y, t),
$$

for each $x, y \in X, t>0$.

On account of formula (1.4) we have, for each $x, y \in X, t>0$, that

$$
P_{e}(x, x, t) \rightarrow_{*} P_{e}(x, y, t)=\frac{e^{-\frac{p(x, y)}{t}}}{e^{-\frac{p(x, x)}{t}}}=e^{-\frac{p(x, y)-p(x, x)}{t}}=e^{-\frac{q_{p}(x, y)}{t}} .
$$

Thus, $Q_{P_{e}}(x, y, t)=e^{-\frac{q_{p}(x, y)}{t}}$, for each $x, y \in X, t>0$.

On the other hand, by Proposition 4.2.6, $\left(X, P_{d}, *_{H}\right)$ is a $G V$-fuzzy partial metric space, where $P_{d}(x, y, t)=\frac{t}{t+p(x, y)}$, for each $x, y \in X, t>0$. Since $*_{H}$ is a continuous Archimedean $t$-norm then, by Theorem 5.2.3, we have that $\left(X, Q_{P_{d}}, *_{H}\right)$ is a $G V$-fuzzy quasi-metric space, where $Q_{P_{d}}$ is given by

$$
Q_{P_{d}}(x, y, t)=P_{d}(x, x, t) \rightarrow_{*} P_{d}(x, y, t),
$$

for each $x, y \in X, t>0$.

On account of formula (1.9) we have, for each $x, y \in X, t>0$, that

$$
\begin{gathered}
P_{d}(x, x, t) \rightarrow_{*} P_{d}(x, y, t)=\frac{\frac{t}{t+p(x, y)} \cdot \frac{t}{t+p(x, x)}}{\frac{t}{t+p(x, y)} \cdot \frac{t}{t+p(x, x)}+\frac{t}{t+p(x, x)}-\frac{t}{t+p(x, y)}}= \\
=\frac{1}{1+\frac{t+p(x, y)}{t}-\frac{t+p(x, x)}{t}}=\frac{t}{t+p(x, y)-p(x, x)}=\frac{t}{t+q_{p}(x, y)} .
\end{gathered}
$$

Thus, $Q_{P_{d}}(x, y, t)=\frac{t}{t+q_{p}(x, y)}$, for each $x, y \in X, t>0$.

Observe that in both examples, the $G V$-fuzzy quasi-metric constructed coincide with the $G V$-fuzzy quasi-metric induced from $q_{p}$. 
To finish this section, we will show that the topology induced by a fuzzy partial metric coincides with the topology induced by the fuzzy quasi-metric constructed in Theorem 5.2.3. To achieve such a goal the next proposition will be helpful.

Proposition 5.2.5. Let $(X, P, *)$ be a $G V$-fuzzy partial metric space, where $*$ is a continuous Archimedean $t$-norm. Then, for each $x, y \in X$, the assignment $\left.\left.P_{x, x, y}:\right] 0, \infty[\rightarrow] 0,1\right]$ given by

$$
\left.P_{x, x, y}(t)=P(x, x, t) \rightarrow_{*} P(x, y, t), \text { for each } t \in\right] 0, \infty[,
$$

is a continuous function.

Proof. Let $(X, P, *)$ be a $G V$-fuzzy partial metric space and suppose that $*$ is a continuous Archimedean $t$-norm. Then, by Theorem 1.1.5 * admits a continuous additive generator $f_{*}$ and attending to formula (1.7) the *residuum $\rightarrow_{*}$ is given by

$$
\left.\left.a \rightarrow_{*} b=f_{*}^{(-1)}\left(\max \left\{f_{*}(b)-f_{*}(a), 0\right\}\right), \text { if } a, b \in\right] 0,1\right] .
$$

Fix $x, y \in X$. Then, the function $P_{x, x, y}$ has the following expression $P_{x, x, y}(t)=f_{*}^{(-1)}\left(\max \left\{f_{*}(P(x, y, t))-f_{*}(P(x, x, t)), 0\right\}\right)$, for each $\left.t \in\right] 0, \infty[$. By axiom $(F P G V 1)$, both $P(x, y, t), P(x, x, t) \in] 0,1]$. Therefore, $P_{x, x, y}$ is continuous due to $f_{*}$ and $f_{*}^{(-1)}$ are continuous and so $P_{x, x, y}$ is the composition of continuous functions.

Let $(X, P, *)$ be a $G V$-fuzzy partial metric, where $*$ is a continuous Archimedean $t$-norm. As a consequence of the preceding proposition, and taking into account Lemma 4.3.5, we have that, for each $x, y \in X$ and $t>0$, the open balls are defined as follows:

$$
B_{P}(x, r, t)=\left\{y \in X: P(x, x, t) \rightarrow_{*} P(x, y, t)>1-r\right\} .
$$


It ensures that $y \in B_{P}(x, r, t)$ if and only if $y \in B_{P_{Q}}(x, r, t)$. Indeed,

$$
\begin{gathered}
y \in B_{P}(x, r, t) \Leftrightarrow P(x, x, t) \rightarrow_{*} P(x, y, t)>1-r \Leftrightarrow \\
\Leftrightarrow Q_{P}(x, y, t)>1-r \Leftrightarrow y \in B_{P_{Q}}(x, r, t) .
\end{gathered}
$$

Hence, $\mathcal{T}(P)=\mathcal{T}\left(Q_{P}\right)$.

\subsection{From $G V$-fuzzy quasi-metrics to $G V$-fuzzy (partial) metrics}

In this section, we tackle the reciprocal of the construction provided in Section 5.2, i.e. we establish a way to construct a $G V$-fuzzy partial metric from a $G V$-fuzzy quasi-metric. To achieve such a goal, we introduce the notion of $G V$-fuzzy weighted quasi-metric adapting the classical notion of weighted quasi-metric to the fuzzy context. Then, we study the topology induced by both, the $G V$-fuzzy quasi-metric and the $G V$-fuzzy partial metric constructed from it.

We begin our study introducing a notion of $G V$-fuzzy weighted quasimetric. This definition is based on the classical definition of weighted quasimetric. Besides, some axioms have been added in order to maintain the "essence" of the George and Veeramani's fuzzification.

Definition 5.3.1. We will say that $(X, Q, *, W)$ is a $G V$-fuzzy weighted quasi-metric space provided that $(X, Q, *)$ is a $G V$-fuzzy quasi-metric space and $W$ is a fuzzy set on $X \times] 0, \infty[$ satisfying, for each $x, y \in X, t \in] 0, \infty[$, the following properties:

(WGV0) $Q(x, y, t) * W(x, t)>0$ 
(WGV1) $Q(x, y, t) * W(x, t)=Q(y, x, t) * W(y, t)$.

(WGV2) The assignment $\left.\left.W_{x}:\right] 0,+\infty[\rightarrow] 0,1\right]$, given by $W_{x}(t)=W(x, t)$ for each $t \in] 0, \infty[$, is a continuous function.

In such a case, the fuzzy set $W$ will be called the fuzzy weight function associated to the $G V$-fuzzy quasi-metric space $(X, Q, *)$.

Moreover, we will say that a $G V$-fuzzy quasi-metric space $(X, Q, *)$ is weightable if there exist a weight function $W: X \times] 0, \infty[$ satisfying axioms $(W G V 0)-(W G V 2)$.

After introducing the above concept we provide, in the next two propositions, examples of $G V$-fuzzy weighted quasi-metric spaces.

Proposition 5.3.2. Let $(X, q, w)$ be a weighted quasi-metric space. Then, $\left(X, Q_{d}, *_{H}, W_{d}\right)$ is a $G V$-fuzzy weighted quasi-metric space, where

$$
\begin{aligned}
Q_{d}(x, y, t) & \left.=\frac{t}{t+q(x, y)}, \text { for each } x, y \in X, t \in\right] 0, \infty[, \\
W_{d}(x, t) & \left.=\frac{t}{t+w(x)} \text { for each } x, y \in X, t \in\right] 0, \infty[,
\end{aligned}
$$

and $*_{H}$ is the Hamacher product $t$-norm.

Proof. On account of Example 5.2.2 (ii), we deduce that $\left(X, Q, *_{H}\right)$ is a $G V$ fuzzy quasi-metric space. So, we just need to show that $W_{d}$ satisfies, for each $x, y \in X$ and $t \in] 0, \infty[$, axioms $(W G V 0)-(W G V 2)$. Then, it remains to show (WKM1).

Let $x, y \in X$ and $t \in] 0, \infty[$. On the one hand,

$$
Q_{d}(x, y, t) *_{H} W_{d}(x, t)=\frac{t}{t+q(x, y)} *_{H} \frac{t}{t+w(x)}=
$$




$$
=\frac{\frac{t}{t+q(x, y)} \cdot \frac{t}{t+w(x)}}{\frac{t}{t+q(x, y)}+\frac{t}{t+w(x)}-\frac{t}{t+q(x, y)} \cdot \frac{t}{t+w(x)}}=\frac{t}{t+q(x, y)+w(x)} .
$$

On the other hand,

$$
\begin{gathered}
Q_{d}(y, x, t) *_{H} W_{d}(y, t)=\frac{t}{t+q(y, x)} *_{H} \frac{t}{t+w(y)}= \\
=\frac{\frac{t}{t+q(y, x)} \cdot \frac{t}{t+w(y)}}{\frac{t}{t+q(y, x)}+\frac{t}{t+w(y)}-\frac{t}{t+q(y, x)} \cdot \frac{t}{t+w(y)}}=\frac{t}{t+q(y, x)+w(y)} .
\end{gathered}
$$

Since $(X, q, w)$ is a weighted quasi-metric space, then $q(x, y)+w(x)=q(y, x)+$ $w(y)$ and so $Q_{d}(x, y, t) *_{H} W_{d}(x, t)=Q_{d}(y, x, t) *_{H} W_{d}(y, t)$.

Following similar arguments to the ones used in the preceding proof, one can show the next proposition.

Proposition 5.3.3. Let $(X, q, w)$ be a weighted quasi-metric space. Then, $\left(X, Q_{e}, *_{P}, W_{e}\right)$ is a $G V$-fuzzy weighted quasi-metric space, where

$$
\begin{gathered}
\left.Q_{e}(x, y, t)=e^{-\frac{q(x, y)}{t}} \text { for each } x, y \in X, t \in\right] 0, \infty[, \\
\left.W_{e}(x, t)=e^{-\frac{w(x)}{t}} \text { for each } x, y \in X, t \in\right] 0, \infty[
\end{gathered}
$$

and $*_{P}$ is the product $t$-norm.

On account of Definition 5.3.1, one can observe that $W$ is defined on $X \times] 0, \infty[$ according to the George and Veeramani's context. The following theorem states a way to obtain a $G V$-fuzzy partial metric from a weighted $G V$-fuzzy quasi-metric.

Theorem 5.3.4. Let $(X, Q, *, W)$ be a $G V$-fuzzy weighted quasi-metric space, where $*$ is a continuous Archimedean $t$-norm. Then, $\left(X, P_{Q}, *\right)$ is a $G V$-fuzzy partial metric space, where $P_{Q}$ is the fuzzy set on $X \times X \times[0, \infty[$ given by:

$$
P_{Q}(x, y, t)=Q(x, y, t) * W(x, t), \text { for each } x, y \in X, t>0 \text {. }
$$


Proof. We will show that every axiom of Definition 4.2.2 is satisfied, for each $x, y, z \in X$ and $t>0$.

(PGV1) Let $x, y \in X$ and $t>0$. On the one hand, since $W$ is a fuzzy weight function, axiom $(W G V 0)$ ensures that $P_{Q}(x, y, t)=Q(x, y, t) *$ $W(x, t)>0$. On the other hand, $P_{Q}(x, y, t)=Q(x, y, t) * W(x, t) \leq$ $Q(x, x, t) * W(x, t)=P(x, x, t)$. Thus, $0<P_{Q}(x, y, t) \leq P_{Q}(x, x, t)$

(PGV2) Obviously, $x=y$ implies $P_{Q}(x, x, t)=P_{Q}(x, y, t)=P_{Q}(y, y, t)$.

Now, suppose that $P_{Q}(x, x, t)=P_{Q}(x, y, t)=P_{Q}(y, y, t)$ for some $x, y \in X, t>0$. Then, on the one hand,

$W(x, t)=Q(x, x, t) * W(x, t)=P_{Q}(x, x, t)=P_{Q}(x, y, t)=Q(x, y, t) * W(x, t)$.

On the other hand,

$W(y, t)=Q(y, y, t) * W(y, t)=P_{Q}(y, y, t)=P_{Q}(x, y, t)=Q(x, y, t) * W(x, t)$.

Besides, since $W$ is a fuzzy weight function, axiom ( $W G V 1)$ ensures that $Q(x, y, t) * W(x, t)=Q(y, x, t) * W(y, t)$. So, $W(y, t)=Q(y, x, t) *$ $W(y, t)$.

Since $*$ is an Archimedean $t$-norm and, $Q(x, y, t)>0$ and $Q(y, x, t)>0$, then $Q(x, y, t)=Q(y, x, t)=1$. Thus, axiom $(Q G V 2)$ implies $x=y$.

(PGV3) Let $x, y \in X$. Since $W$ is a fuzzy weight function, by axiom (WGV1) we have that

$$
P_{Q}(x, y, t)=Q(x, y, t) * w(x, t)=Q(y, x, t) * w(y, t)=P_{Q}(y, x, t) .
$$

(PGV4) Let $x, y, z \in X$ and $t, s>0$. We will see that the following holds:

$$
\begin{aligned}
& P_{Q}(x, x, t+s) \rightarrow_{*} P_{Q}(x, z, t+s) \geq \\
& \left(P_{Q}(x, x, t) \rightarrow_{*} P_{Q}(x, y, t)\right) *\left(P_{Q}(y, y, s) \rightarrow_{*} P_{Q}(y, z, s)\right) .
\end{aligned}
$$


To show it, we claim that $P_{Q}(u, u, r) \rightarrow_{*} P_{Q}(u, v, r)=Q(u, v, r)$, for each $u, v \in X$ and $r>0$.

Fix $u, v \in X$ and $r>0$. First, since $*$ is a continuous Archimedean $t$-norm, there exists an additive generator $f_{*}$ of $*$. Then, using the properties of $f_{*}$ collected in Section 1.1 and taking into account that $Q(u, v, r) * W(u, r)>0$ we have that

$$
\begin{aligned}
P_{Q}(u, u, r) & \rightarrow_{*} P_{Q}(u, v, r)=W(u, r) \rightarrow_{*} Q(u, v, r) * W(u, r) \\
& =f_{*}^{(-1)}\left(\max \left\{f_{*}(Q(u, v, r) * W(u, r))-f_{*}(W(u, r)), 0\right\}\right) \\
& =f_{*}^{(-1)}\left(f_{*}(Q(u, v, r) * W(u, r))-f_{*}(W(u, r))\right) \\
& =f_{*}^{(-1)}\left(f_{*}\left(f_{*}^{(-1)}\left(f_{*}(Q(u, v, r))+f_{*}(W(u, r))\right)\right)-f_{*}(W(u, r))\right) \\
& =f_{*}^{(-1)}\left(f_{*}(Q(u, v, r))+f_{*}(W(u, r))-f_{*}(W(u, r))\right) \\
& =f_{*}^{(-1)}\left(f_{*}(Q(u, v, r))\right) \\
& =f_{*}^{(-1)}\left(f_{*}(Q(u, v, r))=Q(u, v, r) .\right.
\end{aligned}
$$

Therefore, $P_{Q}(u, u, r) \rightarrow_{*} P_{Q}(u, v, r)=Q(u, v, r)$.

Then, $P_{Q}(x, x, t+s) \rightarrow_{*} P_{Q}(x, z, t+s)=Q(x, z, t+s), P_{Q}(x, x, t) \rightarrow_{*}$ $P_{Q}(x, y, t)=Q(x, y, t)$ and $P_{Q}(y, y, s) \rightarrow_{*} P_{Q}(y, z, s)=Q(y, z, s)$. Hence, since $Q$ is a $G V$-fuzzy quasi-metric, axiom ( $Q G V 3)$ ensures that

$$
\begin{aligned}
& P_{Q}(x, x, t+s) \rightarrow_{*} P_{Q}(x, z, t+s) \geq \\
& \left(P_{Q}(x, x, t) \rightarrow_{*} P_{Q}(x, y, t)\right) *\left(P_{Q}(y, y, s) \rightarrow_{*} P_{Q}(y, z, s)\right) .
\end{aligned}
$$

(PGV5) The function $\left(P_{Q}\right)_{x, y}(t)=Q(x, y, t) * W(x, t)$ is continuous because of the continuity of both $Q_{x, y}(t)=Q(x, y, t)$ and $W_{x}(t)=W(x, t)$, and the continuity of the $t$-norm $*$. 
In the next example we will show that the assumption on the $t$-norm, which has to be Archimedean, cannot be removed in Theorem 5.3.4. For that purpose, we introduce the following previous lemma:

Lemma 5.3.5. Let $(X, M, *)$ be a $G V$-fuzzy metric space, where $*$ is integral $t$-norm (i.e. $a * b=0$ if and only if $\min \{a, b\}=0)$. Then $\left(X, Q, *, W_{k}\right)$ is a GV-fuzzy weighted quasi-metric, space where $Q(x, y, t)=M(x, y, t)$ and $W_{k}(x, t)=k$, for every $\left.k \in\right] 0,1[$.

Proof. Let $(X, M, *)$ be a $G V$-fuzzy metric space, where $*$ is continuous integral $t$-norm, and let $k \in] 0,1$. Obviously, every $(X, M, *)$ is a $G V$-fuzzy quasi-metric. So, we need to prove that $W(x, t)=k$ is a fuzzy weight function.

(WGV0) Suppose that $Q(x, y, t) * W_{k}(x, t)=0$ for some $x, y \in X$ and $t>$ 0 . Since $*$ is integral, our assumption implies that $Q(x, y, t)=0$ or $W_{k}(x, t)=0$, which is a contradiction. So, $Q(x, y, t) * W_{k}(x, t)>0$.

(WGV1) Let $x, y \in X$ and $t>0$. By axiom (KM3) we have that $Q(x, y, t)=$ $Q(y, x, t)$, so $Q(x, y, t) * W_{k}(x, t)=Q(x, y, t) * k=Q(y, x, t) * k=$ $Q(y, x, t) * W_{k}(y, t)$.

(WGV2) Obviously, for each $x \in X$ the assignment $\left(W_{k}\right)_{x}$ is a continuous function on $] 0, \infty\left[\right.$, since $\left(W_{k}\right)_{x}(t)=k$ for each $\left.t \in\right] 0, \infty[$.

The previous lemma allows us to introduce the next example.

Example 5.3.6. Let $\left(X, d_{u}\right)$ be metric space, where $X=[0,1]$ and $d_{u}$ is the usual metric of $\mathbb{R}$ restricted to $[0,1]$. Consider the stantard $G V$-fuzzy metric 
deduced from $\left(X, d_{u}\right)$, i.e. $\left(X, M_{d_{u}}, \wedge\right)$, where $\wedge$ is the minimum $t$-norm (see [41]) and

$$
M_{d_{u}}(x, y, t)=\frac{t}{t+d(x, y)}, \text { for each } x, y \in X, t>0 .
$$

Then, by Lemma 5.3.5, $\left(X, Q, \wedge, W_{\frac{1}{2}}\right)$ is a $G V$-fuzzy weighted quasi-metric space, where $Q(x, y, t)=M_{d_{u}}(x, y, t)$ for each $x, y \in X, t>0$. Let $x=1$, $y=0.9$ and $t=10$. We have that

$$
Q(1,0.9,10)=Q(0.9,1,10)=\frac{10}{10+|1-0.9|}=\frac{10}{10.1} \approx 0.99 .
$$

Hence, we have that

$$
\begin{aligned}
Q(1,0.9,10) & \wedge W_{\frac{1}{2}}(1,10)=Q(0.9,1,10) \wedge W_{\frac{1}{2}}(0.9,10)=\min \{0.99,0.5\}=0.5 \\
Q(1,1,10) & \wedge W_{\frac{1}{2}}(1,10)=\min \{1,0.5\}=0.5 \\
Q(0.9,0.9,10) \wedge W_{\frac{1}{2}}(0.9,10) & =\min \{1,0.5\}=0.5
\end{aligned}
$$

Therefore,

$Q(1,0.9,10) \wedge W_{\frac{1}{2}}(1,10)=Q(1,1,10) * w(1,10)=Q(0.9,0.9,10) \wedge W_{\frac{1}{2}}(0.9)$.

If we define $P_{Q}(x, y, t)=Q(x, y, t) \wedge W_{\frac{1}{2}}(x, t)$ then $P_{Q}$ does not fulfil axiom $(P G V 2)$. Indeed, as it has been shown, $P_{Q}(1,0.9,10)=P_{Q}(1,1,10)=$ $P_{Q}(0.9,0.9,10)$ but $1 \neq 0.9$.

As in the preceding section, we motivate Theorem 5.3.4 using the construction provided in it to the examples introduced in Proposition 5.3.2 and 5.3.3.

Let $(X, q, w)$ be a weighted quasi-metric space. Following the Matthews' construction we have that $p_{q}$ is a partial metric on $X$, where $p_{q}(x, y)=$ $q(x, y)-w(x)$ for each $x, y \in X$. 
On the one hand, by Proposition 5.3.2, $\left(X, Q_{d}, *_{H}, W_{d}\right)$ is a $G V$-fuzzy weighted quasi-metric space, where

$$
\begin{aligned}
Q_{d}(x, y, t) & \left.=\frac{t}{t+q(x, y)}, \text { for each } x, y \in X, t \in\right] 0, \infty[, \\
W_{d}(x, t) & \left.=\frac{t}{t+w(x)} \text { for each } x, y \in X, t \in\right] 0, \infty[
\end{aligned}
$$

and $*_{H}$ is the Hamacher product $t$-norm. Since $*_{H}$ is a continuous Archimedean $t$-norm then, by Theorem 5.3.4, we have that $\left(X, P_{Q_{d}}, *_{H}\right)$ is a $G V$-fuzzy partial metric space, where $P_{Q_{d}}$ is given by

$$
P_{Q_{d}}(x, y, t)=Q_{d}(x, y, t) *_{H} W_{d}(x, t)
$$

for each $x, y \in X, t>0$.

Then, for each $x, y \in X, t>0$, we have that

$$
\begin{gathered}
Q_{d}(x, y, t) *_{H} W_{d}(x, t)=\frac{\frac{t}{t+q(x, y)} \cdot \frac{t}{t+w(x)}}{\frac{t}{t+q(x, y)}+\frac{t}{t+w(x)}-\frac{t}{t+q(x, y)} \cdot \frac{t}{t+w(x)}}= \\
=\frac{t}{t+q(x, y)+w(x)}=\frac{t}{t+p_{q}(x, y)} .
\end{gathered}
$$

Thus, $P_{Q_{d}}(x, y, t)=\frac{t}{t+p_{q}(x, y)}$, for each $x, y \in X, t>0$.

On the other hand, by Proposition 5.3.3, $\left(X, Q_{e}, *_{P}, W_{e}\right)$ is a $G V$-fuzzy weighted quasi-metric space, where

$$
\begin{gathered}
\left.Q_{e}(x, y, t)=e^{-\frac{q(x, y)}{t}} \text { for each } x, y \in X, t \in\right] 0, \infty[, \\
\left.W_{e}(x, t)=e^{-\frac{w(x)}{t}} \text { for each } x, y \in X, t \in\right] 0, \infty[
\end{gathered}
$$

and $*_{P}$ is the product $t$-norm. Since $*_{P}$ is a continuous Archimedean $t$-norm then, by Theorem 5.2.3, we have that $\left(X, P_{Q_{e}}, *_{P}\right)$ is a $G V$-fuzzy partial metric space, where $P_{Q_{e}}$ is given by

$$
P_{Q_{e}}(x, y, t)=Q_{e}(x, y, t) *_{P} W_{e}(x, t),
$$


for each $x, y \in X, t>0$.

Then, for each $x, y \in X, t>0$, we have that

$$
Q_{e}(x, y, t) *_{P} W_{e}(x, t)=e^{-\frac{q(x, y)}{t}} \cdot e^{-\frac{w(x)}{t}}=e^{-\frac{q(x, y)+w(x)}{t}}=e^{-\frac{p_{q}(x, y)}{t}} .
$$

Thus, $P_{Q_{e}}(x, y, t)=e^{-\frac{p_{q}(x, y)}{t}}$, for each $x, y \in X, t>0$.

Observe that in both examples, the $G V$-fuzzy partial metric constructed coincide with the $G V$-fuzzy partial metric induced from $p_{q}$.

Now, we approach the study on the relationship between the topologies induced by a $G V$-fuzzy weighted quasi-metric and the $G V$-fuzzy partial metric constructed from it.

Let $(X, Q, *, W)$ be a $G V$-fuzzy quasi-metric space, where $*$ is a continuous Archimedean $t$-norm. On the one hand, for each $x \in X, r \in] 0,1[$ and $t>0$, we have that

$$
B_{Q}(x, r, t)=\{y \in X: Q(x, y, t)>1-r\} .
$$

On the other hand, by Proposition 5.2.5 and Lemma 4.3.5 we have that

$$
B_{P_{Q}}(x, r, t)=\left\{y \in X: P_{Q}(x, x, t) \rightarrow_{*} P_{Q}(x, y, t)>1-r\right\},
$$

for each $x \in X, r \in] 0,1[$ and $t>0$.

Moreover, in the demonstration of Theorem 5.3.4, $P_{Q}(x, x, t) \rightarrow_{*} P_{Q}(x, y, t)=$ $Q(x, y, t)$. Thus, it is obvious that, for each $x \in X, r \in] 0,1[$ and $t>0$, $y \in B_{Q}(x, r, t)$ if and only if $y \in B_{P_{Q}}(x, r, t)$. Hence, $\mathcal{T}(Q)=\mathcal{T}\left(P_{Q}\right)$.

To finish this section we tackle a question related with the construction given in Theorem 5.2.3. In such a theorem, we provide a way of obtaining a $G V$-fuzzy quasi-metric from a $G V$-partial one. It is based on the results 
given by Matthews in [6] for the classical case. Taking into account that, in the construction of Matthews, the obtained quasi-metric from a partial one turns out to be weightable, we wonder it it is so in the fuzzy context. The next theorem answer affirmatively such a question.

Theorem 5.3.7. Let $(X, P, *)$ be a $G V$-fuzzy partial metric space, where * is a continuous Archimedean t-norm. Then, $\left(X, Q_{P}, *, W_{P}\right)$ is a $G V$-fuzzy weighted quasi-metric space, where

$$
\left.Q_{P}(x, y, t)=P(x, x, t) \rightarrow_{*} P(x, y, t) \text { for each } x, y \in X, t \in\right] 0, \infty[
$$

and

$$
\left.W_{P}(x, t)=P(x, x, t) \text { for each } x \in X, t \in\right] 0, \infty[\text {. }
$$

Proof. Let $(X, P, *)$ be a $G V$-fuzzy partial metric space, where $*$ is a continuous Archimedean $t$-norm. Theorem 5.2.3 ensures that $\left(X, Q_{P}, *\right)$ is a $G V$-fuzzy quasi-metric space. So, we just need to show that $W_{P}$ satisfies, for each $x, y \in X$ and $t>0$, axioms (WGV0), (WGV1) and (WGV2).

First, observe that $*$ is a continuous Archimedean $t$-norm, so there exists a continuous additive generator $f_{*}$ of $*$. Now, fix $x, y \in X$ and $t>0$ :

(WGV0) $Q_{P}(x, y, t) * W_{P}(x, t)=\left(P(x, x, t) \rightarrow_{*} P(x, y, t)\right) * P(x, x, t)$. By definition of additive generator and taking into account formula (1.7), since $P(x, x, t) \geq P(x, y, t)>0$ by axiom $(P G V 1)$, we have that

$$
\begin{gathered}
\left(P(x, x, t) \rightarrow_{*} P(x, y, t)\right) * P(x, x, t)= \\
=f_{*}^{(-1)}\left(f_{*}\left(P(x, x, t) \rightarrow_{*} P(x, y, t)\right)+f_{*}(P(x, x, t))\right)= \\
=f_{*}^{(-1)}\left(f _ { * } \left(f^{(-1)}\left(f_{*}(P(x, y, t))-f_{*}(P(x, x, t))\right)+f_{*}(P(x, x, t))=\right.\right. \\
=f_{*}^{(-1)}\left(f_{*}(P(x, y, t))-f_{*}(P(x, x, t))+f_{*}(P(x, x, t))=\right.
\end{gathered}
$$




$$
=f_{*}^{(-1)}\left(f_{*}(P(x, y, t))\right)=P(x, y, t) .
$$

Hence, $Q_{P}(x, y, t) * W_{P}(x, t)=\left(P(x, x, t) \rightarrow_{*} P(x, y, t)\right) * P(x, x, t)=$ $P(x, y, t)>0$.

(WGV1) As it was exposed above, $Q_{P}(x, y, t) * W_{P}(x, t)=P(x, y, t)$. Analogously, $Q_{P}(y, x, t) * W_{P}(y, t)=P(y, x, t)$. By axiom $(P G V 3)$ we have that $P(x, y, t)=P(y, x, t)$. Therefore, $Q_{P}(x, y, t) * W_{P}(x, t)=$ $P(x, y, t)=P(y, x, t)=Q_{P}(y, x, t) * W_{P}(y, t)$.

(WGV2) By axiom $(P G V 5)$ we have that the assignment $\left.P_{x, x}:\right] 0, \infty[\rightarrow$ ] $0,1]$ is a continuous function. Thus, since $\left(W_{P}\right)_{x}(t)=P_{x, x}(t)$ for each $t \in]\left[0, \infty\left[\right.\right.$ then, the assignment $\left.\left.\left(W_{P}\right)_{x}:\right] 0, \infty[\rightarrow] 0,1\right]$ is a continuous function. 


\section{Chapter 6}

\section{Discussion of the obtained results and conclusions}

In Chapter 2 we have introduced and studied the concept of extended fuzzy metric $M^{0}$. We have constructed a topology deduced from an extended fuzzy metric and we have characterized convergent sequences in it. We also have approached the relationship between $s$-convergence and $\tau_{M^{0}}$-convergence. Furthermore, we have studied Cauchyness and completeness in extended fuzzy metrics. Finally, we have obtained a fixed point theorem in extended fuzzy metric spaces.

In Chapter 3 we have given an example of a $\psi$-contractive sequence which is not Cauchy. Then we have introduced and studied a notion of strictly contractive sequence, and we have corrected in an appropriate way the proof of Lemma 3.2 of [12].

In Chapter 4 we have introduced a notion of fuzzy partial metric space 
$(X, P, *)$ which constitutes an adaptation of the notion of partial metric space given in [6] to the fuzzy setting. Such an adaptation is based on the fuzzy metric space given in [29], using the residuum operator $\rightarrow_{*}$ associated to a continuous $t$-norm $*$, without any extra condition on $*$. Then, we have constructed a topology $\mathcal{T}_{P}$ on $X$, deduced from $P$, and we have proved that $\left(X, \mathcal{T}_{P}\right)$ is a $T_{0}$-space. The introduced notion of fuzzy partial metric opens several lines of research in the fuzzy setting. On the one hand, the study of the (fuzzy partial) metric properties, and in particular fixed point theory. On the other hand, its applicability to engineering problems in which it makes sense to consider that the self-similarity can be less than 1 .

In Chapter 5 we establish a duality between $G V$-fuzzy partial metrics defined in Chapter 4 and $G V$-fuzzy quasi-metrics defined in [24], by defining the concept of $G V$-fuzzy weighted quasi-metric, which is a generalization of classical weighted quasi-metrics. This duality is motivated by the classical relationship between partial metrics and quasi-metrics introduced by Matthews in [6]. 


\section{Bibliography}

[1] L. A. Ricarte and S. Romaguera, A domain-theoretic approach to fuzzy metric spaces, Topology and its Applications 163 (2014), 149-159.

[2] M. Bukatin, R. Kopperman, and S. Matthews, Some corollaries of the correspondence between partial metrics and multivalued equalities, Fuzzy Sets and Systems 256 (2014), 57-72.

[3] G. Constantin and I. Istratescu, Elements of probabilistic analysis with applications, Kluwer Academic Publishers \& Bucuresti: Editura Academiei (1989).

[4] M. Demirci, The order-theoretic duality and relations between partial metrics and local equalities, Fuzzy Sets and Systems 192 (2012), 45-57.

[5] J. G. Camarena, V. Gregori, S. Morillas, and A. Sapena, Fast detection and removal of impulsive noise using peer groups and fuzzy metrics, Journal of Visual Communication and Image Representation 19 (1) (2008), 20-29.

[6] S. G. Matthews, Partial metric topology, Annals of the New York Academy of Sciences 728 (1994), 183-197. 
[7] A. George and P. Veeramani, On some results in fuzzy metric spaces, Fuzzy Sets and Systems 64 (1994), 395-399.

[8] A. George and P. Veeramani, Some theorems in fuzzy metric spaces, J. Fuzzy Math 3 (1995), 933-940.

[9] M. Grabiec, Fixed points in fuzzy metric spaces, Fuzzy Sets and Systems 27 (1989), 385-389.

[10] M. Grabiec, Y. Je Cho, and V. Radu, On nonsymmetric topological and probabilistic structures, Nova Science Publishers, Inc., New York (2006).

[11] V. Gregori and J. J. Miñana, std-convergence in fuzzy metric spaces, Fuzzy Sets and Systems 267 (2015), 140-143.

[12] V. Gregori and J. J. Miñana, On fuzzy $\psi$-contractive sequences and fixed point theorems, Fuzzy Sets and Systems 300 (2016), 93-101.

[13] V. Gregori, J. J. Miñana, and D. Miravet, Contractive sequences in fuzzy metric spaces, Fuzzy Sets and Systems (2019), http://dx.doi.org/10.1016/j.fss.2019.01.003.

[14] V. Gregori, J. J. Miñana, and D. Miravet, Extended fuzzy metrics and fixed point theorems, Mathematics 303:7 (2019).

[15] V. Gregori, J. J. Miñana, and D. Miravet, Fuzzy partial metric spaces, International Journal of General Systems 48:3 (2019), 260-279.

[16] V. Gregori, J. J. Miñana, and S. Morillas, Some questions in fuzzy metric spaces, Fuzzy Sets and Systems 204 (2012), 71-85.

[17] V. Gregori, J. J. Miñana, and S. Morillas, A note on convergence in fuzzy metric spaces, Iranian Journal of Fuzzy Systems 11 (2014), 75-85.

[18] V. Gregori, J. J. Miñana, S. Morillas, and A. Sapena, Cauchyness and convergence in fuzzy metric spaces, RACSAM 111 (2017), 25-37. 
[19] V. Gregori, J. J. Miñana, and A. Sapena, On a class of completable fuzzy metric spaces, Fuzzy Sets and Systems 161:16 (2010), 2193-2205.

[20] V. Gregori, A. López-Crevillén, S. Morillas, and A. Sapena, On convergence in fuzzy metric spaces, Topology and its Applications 156 (2009), 3002-3006.

[21] V. Gregori and S. Romaguera, Some properties of fuzzy metric spaces, Fuzzy Sets and Systems 115 (2000), 485-489.

[22] V. Gregori and S. Romaguera, On completion of fuzzy metric spaces, Fuzzy Sets and Systems 130 (2002), 399-404.

[23] V. Gregori and S. Romaguera, Characterizing completable fuzzy metric spaces, Fuzzy Sets and Systems 144 (2004), 411-420.

[24] V. Gregori and S. Romaguera, Fuzzy quasi-metric-spaces, Applied General Topology 5 (2004), 129-136.

[25] V. Gregori and A. Sapena, On fixed-point theorems in fuzzy metric spaces, Fuzzy Sets and Systems 125 (2002), 245-252.

[26] J. Gutiérrez-García, S. Romaguera, and M. Sanchís, Fuzzy uniform structures and continuous t-norms, Fuzzy Sets and Systems 161 (7) (2010), 1011-1021.

[27] O. Hadzic and E. Pap, The fixed point theory in probabilistic metric spaces, Kluwer Academic Publishers (2001).

[28] V. Istratescu, An introduction to theory of probabilistic metric spaces, with applications, Ed. Tehnica, Bucuresti (1974).

[29] K. Kramosil and J. Michalek, Fuzzy metric and statistical metric spaces, Kybernetika 11 (1975), 336-344. 
[30] C. L. Chang, Fuzzy topological spaces, Journal of Mathematical Analysis 24 (1968), 182-190.

[31] F. Mehmood, R. Ali, C. Ionescu, and T. Kamran, Extended fuzzy bmetric spaces, J. Math. Anal. 8:6 (2017), 124-131.

[32] K. Menger, Statistical metrics, Proc. Nat. Acad. Sci., U.S.A. 28 (1942), $535-537$.

[33] D. Mihet, A banach contraction theorem in fuzzy metric spaces, Fuzzy Sets and Systems 144 (2004), 431-439.

[34] D. Mihet, On fuzzy contractive mappings in fuzzy metric spaces, Fuzzy Sets and Systems 158 (2007), 915-921.

[35] D. Mihet, Fuzzy $\psi$-contractive mappings in non-archimedean fuzzy metric spaces, Fuzzy Sets and Systems 159 (2008), 739-744.

[36] D. Mihet, A note on fuzzy contractive mappings in fuzzy metric spaces, Fuzzy Sets and Systems 251 (2014), 83-91.

[37] E. P. Klement, R. Mesiar, and E. Pap, Triangular norms, Springer Netherlands (2000).

[38] V. Radu, Some remarks on the probabilistic contractions on fuzzy menger spaces, Automation Computers Applied Mathematics 11 (2002), 125131.

[39] J. Rodríguez-López and S. Romaguera, The Hausdorff fuzzy metric on compact sets, Fuzzy Sets and Systems 147 (2004), 273-283.

[40] S. Romaguera and P. Tirado, Contraction maps on Ifgm-spaces with application to Recurrence Equations of Quicksort, Electronic Notes in Theoretical Computer Science 225 (2009), 339-342. 
[41] A. Sapena, A contribution to the study of fuzzy metric spaces, Applied General Topology 2 (2001), 63-76.

[42] B. Schweizer and A. Sklar, Probabilistic metric spaces, North Holland Series in Probability and Applied Mathematics, New York, Amsterdam, Oxford (1983).

[43] S. Sedghi, N. Shobkolaei, and I. Altun, Partial fuzzy metric space and some fixed point results, Communications in Mathematics 23 (2015), $131-142$.

[44] H. Sherwood, On the completion of probabilistic metric spaces, Z. Wahrschein-lichkeitstheoric verw. Geb 6 (1966), 62-64.

[45] H. Sherwood, Complete probabilistic metric spaces, Z. Wahrscheinlichkeitstheoric verw. Geb 20 (1971), 117-128.

[46] S. Shukla, D. Gopal, and W. Sintunavarat, A new class of fuzzy contractive mappings and fixed point theorems, Fuzzy Sets and Systems 350 (2018), 85-94.

[47] R. Vasuki and P. Veeramani, Fixed point theorems and cauchy sequences in fuzzy metric spaces, Fuzzy Sets and Systems 135 (2003), 415-417.

[48] P. Veeramani, Best approximation in fuzzy metric spaces, Journal of Fuzzy Mathematics 9 (201), 75-80.

[49] D. Wardowski, Fuzzy contractive mappings and fixed points in fuzzy metric spaces, Fuzzy Sets and Systems 222 (2013), 108-114.

[50] J. Wu and Y. Yue, Formal balls in fuzzy partial metric spaces, Iranian Journal of Fuzzy Systems 14 (2017), 155-164.

[51] M. Ying, A new approach for fuzzy topology (i), Fuzzy Sets and Systems 39 (1991), 303-321. 
[52] Y. Yue, Separated $\delta^{+}$-valued equivalences as probabilistic partial metric spaces, Journal of Intelligent and Fuzzy Systems 28 (2015), 2715-2724.

[53] Y. Yue and M. Gu, Fuzzy partial (pseudo-)metric spaces, Journal of Intelligent and Fuzzy Systems 27 (2014), 1153-1159.

[54] L. Zadeh, Fuzzy sets, Information and Control 8 (1965), 338-353. 